\title{
The Ekman flow and related problems: spectral theory and numerical analysis
}

\author{
BY LEON GREENBERG
}

Dept. of Mathematics, University of Maryland, College Park, Maryland, MD 20740, U.S.A.

AND MARCO MARLETTA

Dept. of Mathematics and Computer Science, University of Leicester, University Road, Leicester LE1 $7 R H$.

(Received 13 June 2002; revised 14 October 2002)

\section{Abstract}

We consider singular block operator problems of the type arising in the study of stability of the Ekman boundary layer. The essential spectrum is located, and an analysis of the $L^{2}$ solutions of a related first order system of differential equations allows the development of a Titchmarsh-Weyl coefficient $M(\lambda)$. This, in turn, permits a rigorous analysis of the convergence of approximations to the spectrum arising from regular problems. Numerical results illustrate the theory.

\section{Introduction}

Recently, a number of papers have appeared on the spectral analysis of block operators (cf. $[\mathbf{1}, \mathbf{2}, \mathbf{4}, \mathbf{1 5}]$ ). This work has its origin and motivation in the analysis of stability in fluid mechanics and magnetohydrodynamics (ef. $[\mathbf{9}, \mathbf{1 9}, 27])$.

In this paper, our first goal is to determine conditions under which a block operator (whose blocks are ordinary differential operators) can be reduced to a system of first order ODEs. We give some examples where this is not the case; and Theorem $2 \cdot 1$ gives sufficient conditions for this to be true.

Our main goal is the spectral analysis of the block operator which arises in the study of stability of the Ekman boundary layer (cf. Faller [12], Lilly [20]):

$$
\begin{array}{rr}
\left(\begin{array}{cc}
\left(-D^{2}+\alpha^{2}\right)^{2}+i \alpha R V\left(-D^{2}+\alpha^{2}\right)+i \alpha R V^{\prime \prime} & 2 D \\
2 D+i \alpha R U^{\prime} & \left(-D^{2}+\alpha^{2}\right)+i \alpha R V
\end{array}\right)\left(\begin{array}{l}
y \\
z
\end{array}\right) \\
=\lambda\left(\begin{array}{cc}
-D^{2}+\alpha^{2} & 0 \\
0 & I
\end{array}\right)\left(\begin{array}{l}
y \\
z
\end{array}\right)
\end{array}
$$

with boundary conditions

$$
\begin{gathered}
y(0)=y^{\prime}(0)=z(0)=0, \\
y(\infty)=y^{\prime \prime}(\infty)=z(\infty)=0 .
\end{gathered}
$$

Here, $\alpha$ is the wave number, $R$ is the Reynolds number, and $U, V$ are known smooth 
functions. For the operator theory which we develop later, the conditions at $\infty$ have to be stated more rigorously. We shall see that the conditions required are $y \in H^{4}[0, \infty)$ and $z \in H^{2}[0, \infty)$, where $H^{p}[0, \infty)$ is the usual Sobolev space of functions whose (weak) derivatives of orders $0,1, \ldots, p$ lie in $L^{2}[0, \infty)$. Equation (1.1) is equivalent to a system of first order ODEs.

Throughout this paper we shall assume that the functions $U, V$ satisfy the following conditions:

(A1-1) $U \in C^{1}[0, \infty), V \in C^{2}[0, \infty)$;

(A1.2) $\lim _{x \rightarrow \infty} U^{\prime}(x)=\lim _{x \rightarrow \infty} V(x)=\lim _{x \rightarrow \infty} V^{\prime \prime}(x)=0$;

(A1·3) $U^{\prime}, V, V^{\prime \prime} \in L^{1}[0, \infty)$.

The paper is set out as follows. Section 2 deals with general $2 \times 2$ block operators, and conditions are found for the eigenvalue problem to be equivalent to a system of first order ODEs. In Section 3 we find the essential spectrum for the Ekman problem. Section 4 discusses the $L^{2}$ and non- $L^{2}$ solutions. In Section 5 we show that all eigenvalues are located in a certain semi-infinite strip. Section 6 investigates the possible existence of an open set of eigenvalues. We need to know that such sets do not exist in order to construct the Titchmarsh-Weyl $M(\lambda)$ matrices in Section 7. We show that the truncated problem on a finite interval $[0, X]$ (with additional boundary conditions at $X$ ) has no open sets of eigenvalues. Furthermore, for generic Reynolds numbers, the Ekman problem on $[0, \infty)$ has no open sets of eigenvalues. (More precisely, the set of Reynolds numbers which admit such sets has no accumulation points.) The nonexistence of open sets of eigenvalues for all Reynolds numbers remains an open question. In Section 8, assuming that there are no open sets of eigenvalues, we use $M(\lambda)$ to show that the eigenvalues of the truncated problems converge to the eigenvalues of the Ekman problem (and only to those eigenvalues). In Section 9 we discuss the shooting method that we use to approximate the eigenvalues. We have developed a package of subroutines SLNSA for solving nonselfadjoint eigenvalue problems. In Section 10 we calculate the neutral curve and critical Reynolds number (for certain parameters of the problem), and compare with results of Lilly [20].

\section{Transformation of block operator problems to systems of ODEs}

We shall consider spectral problems for $2 \times 2$ matrix operators of the form

$$
\left(\begin{array}{ll}
A & B \\
C & D
\end{array}\right)\left(\begin{array}{l}
y \\
z
\end{array}\right)=\lambda\left(\begin{array}{l}
y \\
z
\end{array}\right)
$$

or more generally

$$
\left(\begin{array}{ll}
A & B \\
C & D
\end{array}\right)\left(\begin{array}{l}
y \\
z
\end{array}\right)=\lambda\left(\begin{array}{cc}
M & N \\
P & Q
\end{array}\right)\left(\begin{array}{l}
y \\
z
\end{array}\right),
$$

where the blocks $A, B, C, D, M, N, P, Q$ are ordinary differential operators. Here, $\lambda$ is a complex parameter, and for simplicity, we suppose that $y(x)$ and $z(x)$ are complex, scalar functions defined on a finite interval $[a, b]$. (The calculations and results of this section are valid for vector functions as well. Later, finite intervals will play the role of truncations of the infinite interval $[0, \infty)$.) If we set $A(\lambda)=$ $A-\lambda M, B(\lambda)=B-\lambda N, C(\lambda)=C-\lambda P, D(\lambda)=D-\lambda Q$, then (2.2) has the 
form

$$
\begin{aligned}
& A(\lambda) y+B(\lambda) z=0 \\
& C(\lambda) y+D(\lambda) z=0 .
\end{aligned}
$$

The block operator problem consists of these equations, together with $2 n$ independent, separated boundary conditions for $y$, and $2 m$ independent, separated boundary conditions for $z$ of the form:

$$
\begin{array}{ll}
\sum_{\beta=0}^{2 n-1} p_{\alpha \beta} y^{(\beta)}(a)=0=\sum_{\beta=0}^{2 n-1} q_{\alpha \beta} y^{(\beta)}(b) & (1 \leqslant \alpha \leqslant n), \\
\sum_{\nu=0}^{2 m-1} r_{\mu \nu} z^{(\nu)}(a)=0=\sum_{\nu=0}^{2 m-1} s_{\mu \nu} z^{(\nu)}(b) & (1 \leqslant \mu \leqslant m),
\end{array}
$$

where the coefficients $p_{\alpha \beta}, q_{\alpha \beta}, r_{\mu \nu}, s_{\mu \nu}$ are complex constants. The differential operators are assumed to have the form:

$$
\begin{aligned}
& A(\lambda) y=a_{0}(x) y^{(2 n)}+a_{1}(x, \lambda) y^{(2 n-1)}+\cdots+a_{2 n}(x, \lambda) y, \\
& B(\lambda) z=b_{0}(x, \lambda) z^{(k)}+b_{1}(x, \lambda) z^{(k-1)}+\cdots+b_{k}(x, \lambda) z, \\
& C(\lambda) y=c_{0}(x, \lambda) y^{(h)}+c_{1}(x, \lambda) y^{(h-1)}+\cdots+c_{h}(x, \lambda) y, \\
& D(\lambda) z=d_{0}(x, \lambda) z^{(2 m)}+d_{1}(x, \lambda) z^{(2 m-1)}+\cdots+d_{2 m}(x, \lambda) z,
\end{aligned}
$$

and to satisfy the following assumptions:

(A2·1) $h<2 n, h+k \leqslant 2(m+n)$;

(A2-2) the coefficients $a_{i}, b_{j}, c_{p}, d_{q}$, which are complex functions, are analytic in $\lambda$ for each $x$ and are at least $|k-2 m|$ times differentiable with respect to $x$;

(A2-3) $a_{0}(x) \neq 0$; and if $m>0$, then $d_{0}(x, \lambda)=d_{0}(x) \neq 0$;

(A2.4) if $h+k=2(m+n)$, then $a_{0}(x) d_{0}(x, \lambda)-b_{0}(x, \lambda) c_{0}(x, \lambda)$ has only isolated zeros for each value of $\lambda$. In particular, it is not identically zero.

Under these assumptions, we shall show that the problem $(2 \cdot 3-2 \cdot 6)$ is equivalent to a system of first order ODEs with separated boundary conditions. However the coefficients of this first order system may have singularities.

Example 1. To illustrate the nature of the singularities in the coefficients of the ODEs, consider the problem:

$$
\left(\begin{array}{cc}
-D^{2} & 1 \\
q(x) & u(x)
\end{array}\right)\left(\begin{array}{l}
y \\
z
\end{array}\right)=\lambda\left(\begin{array}{l}
y \\
z
\end{array}\right)
$$

where $D=d / d x$, and with boundary conditions $y(0)=0=y(1)$. This is equivalent to the problem

$$
\left\{\begin{array}{l}
y_{1}^{\prime}=y_{2} \\
y_{2}^{\prime}=\left(\frac{u(x)}{\lambda-q(x)}-\lambda\right) y_{1},
\end{array}\right.
$$

with boundary conditions $y_{1}(0)=0=y_{1}(1)$.

Remark 1. Assumption (A2.4) implies that $A(\lambda) y+B(\lambda) z$ cannot be a linear combination of $C(\lambda) y+D(\lambda) z$ and its derivatives. For if it were, and if 
$f(x) d^{j} / d x^{j}(C(\lambda) y+D(\lambda) z)$ is the highest derivative in the linear combination, then $2 n=h+j$ and $k=2 m+j$, so that $h+k=h+2 m+j=2 m+2 n$. Furthermore, $a_{0}=f c_{0}$ and $b_{0}=f d_{0}$, which implies that $a_{0} d_{0}-b_{0} c_{0}$ is identically zero. If $A(\lambda) y+B(\lambda) z$ is a linear combination of $C(\lambda) y+D(\lambda) z$ and its derivatives, then the system is determined by $C(\lambda) y+D(\lambda) z$, and can be expected to have strange behaviour, as illustrated by the following two examples.

Example 2. This is an example where $A(\lambda) y+B(\lambda) z$ is a derivative of $C(\lambda) y+$ $D(\lambda) z$. Consider the problem

$$
\left(\begin{array}{cc}
D^{4} & D^{5} \\
D & D^{2}
\end{array}\right)\left(\begin{array}{l}
y \\
z
\end{array}\right)=\lambda\left(\begin{array}{cc}
D^{3} & D^{4} \\
1 & D
\end{array}\right)\left(\begin{array}{l}
y \\
z
\end{array}\right),
$$

with boundary conditions $y(0)=y^{\prime}(0)=0=y(1)=y^{\prime}(1), z(0)=0=z(1)$. Here,

$$
\begin{aligned}
& A(\lambda) y+B(\lambda) z=y^{(4)}-\lambda y^{\prime \prime \prime}+z^{(5)}-\lambda z^{(4)}=0, \\
& C(\lambda) y+D(\lambda) z=y^{\prime}-\lambda y+z^{\prime \prime}-\lambda z^{\prime}=0 .
\end{aligned}
$$

If we choose any $z \in C^{\infty}[0,1]$, such that $z^{(j)}(0)=0=z^{(j)}(1)$ for $0 \leqslant j \leqslant 2$, and set $y=-z^{\prime}$, then $(y, z)^{T}$ is an eigenfunction for all $\lambda \in \mathbb{C}$. Thus every $\lambda$ is an eigenvalue with infinite multiplicity.

Example 3. In this example, assumption (A4) is violated, but $A(\lambda) y+B(\lambda) z$ is not a linear combination of $C(\lambda) y+D(\lambda) z$ and its derivatives. Consider the problem

$$
\left(\begin{array}{cc}
D^{2} & D \\
D & 1
\end{array}\right)\left(\begin{array}{l}
y \\
z
\end{array}\right)=\lambda\left(\begin{array}{cc}
2 D & 2 \\
1 & 0
\end{array}\right)\left(\begin{array}{l}
y \\
z
\end{array}\right),
$$

with boundary conditions $y(0)=0=y(1)$. Here,

$$
\begin{aligned}
& A(\lambda) y+B(\lambda) z=y^{\prime \prime}-2 \lambda y^{\prime}+z^{\prime}-2 \lambda z=0, \\
& C(\lambda) y+D(\lambda) z=y^{\prime}-\lambda y+z=0 .
\end{aligned}
$$

For $\lambda=0$, these equations are:

$$
\begin{aligned}
y^{\prime \prime}+z^{\prime} & =0, \\
y^{\prime}+z & =0 .
\end{aligned}
$$

If we choose any function $y \in C^{\infty}[0,1]$ such that $y(0)=0=y(1)$, and set $z=-y^{\prime}$, then (2.9) is satisfied, so $(y, z)^{T}$ is an eigenfunction for $\lambda=0$. Thus $\lambda=0$ is an eigenvalue with infinite multiplicity. If $\lambda \neq 0$, then the first equation of $(2 \cdot 8)$ together with the derivative of the second equation imply that $z=-\frac{1}{2} y^{\prime}$. Substituting this into the second equation of $(2 \cdot 8)$, we obtain $y^{\prime}=2 \lambda y$. Therefore $y=c \exp (2 \lambda x)$, and we see that the only solution is $(y, z)=(0,0)$. Thus the only eigenvalue is $\lambda=0$.

Since Examples 2 and 3 have eigenvalues with infinite multiplicity, neither problem can be equivalent to a system of first order ODEs with separated boundary conditions.

In the following, we assume that conditions (A2·1)-(A2·4) are satisfied.

Lemia $2 \cdot 1$. If $k \geqslant 2 m$, then equations $(2 \cdot 3),(2 \cdot 4)$ can be transformed to an equivalent system:

$$
\begin{aligned}
& \hat{A}(\lambda) y+\hat{B}(\lambda) z=0, \\
& C(\lambda) y+D(\lambda) z=0 .
\end{aligned}
$$


Where

$$
\begin{aligned}
& \hat{A}(\lambda) y=a_{0}(x) y^{(2 n)}+\hat{a}_{1}(x, \lambda) y^{(2 n-1)}+\cdots+\hat{a}_{2 n}(x, \lambda) y, \\
& \hat{B}(\lambda) z=\hat{b}_{0}(x, \lambda) z^{(\hat{k})}+\hat{b}_{1}(x, \lambda) z^{(2 m-1)}+\cdots+\hat{b}_{\hat{k}}(x, \lambda) z
\end{aligned}
$$

and $\hat{k}<m$ if $m>0$; moreover, $\hat{B}(\lambda) z=0$ (i.e. $z$ does not appear in the first equation) if $m=0$. The coefficients of $\hat{A}(\lambda)$ and $\hat{B}(\lambda)$ may have singularities. However, if $m>0$ and $h+k<2(m+n)$, then there are no singularities.

Proof. The equations $(2 \cdot 3),(2 \cdot 4)$ have the form:

$$
\begin{gathered}
a_{0} y^{(2 n)}+a_{1} y^{(2 n-1)}+\cdots a_{2 n} y+b_{0} z^{(k)}+\cdots+b_{k} z=0, \\
c_{0} y^{(h)}+c_{1} y^{(h-1)}+\cdots c_{h} y+d_{0} z^{(2 m)}+\cdots+d_{2 m} z=0 .
\end{gathered}
$$

Dividing $(2 \cdot 11)$ by $d_{0}$ and differentiating $p$ times, which we may do for any $p \leqslant|k-2 m|$ by Assumption (A2.2), we obtain a sequence of equations:

$$
\begin{aligned}
& \gamma_{0} y^{(h)}+\gamma_{0,1} y^{(h-1)}+\cdots+\gamma_{0, h} y+z^{(2 m)}+\delta_{0,1} z^{(2 m-1)}+\cdots+\delta_{0,2 m} z=0, \\
& \gamma_{0} y^{(h+1)}+\gamma_{1,0} y^{(h)}+\cdots+\gamma_{1, h} y+z^{(2 m+1)}+\delta_{1,1} z^{(2 m-1)}+\cdots+\delta_{1,2 m} z=0, \\
& \gamma_{0} y^{(h+2)}+\tilde{\gamma}_{2,1} y^{(h+1)}+\gamma_{2,0} y^{(h)}+\cdots+\gamma_{2, h} y+z^{(2 m+2)}+\delta_{2,1} z^{(2 m-1)}+\cdots+\delta_{2,2 m} z=0, \\
& \vdots \\
& \gamma_{0} y^{(h+p)}+\tilde{\gamma}_{p, 1} y^{(h+p-1)}+\cdots+\tilde{\gamma}_{p, p-1} y^{(h+1)}+\gamma_{p, 0} y^{(h)}+\cdots+\gamma_{p, h} y+z^{(2 m+p)}+\delta_{p, 1} z^{(2 m-1)}+\cdots+\delta_{p, 2 m} z=0 .
\end{aligned}
$$

The coefficient $\gamma_{0}=c_{0} / d_{0}$. By Assumption $(\mathrm{A} 2 \cdot 3)$, if $m>0$, then $d_{0}=d_{0}(x) \neq 0$, and $\gamma_{0}$ is non-singular. The term $z^{(2 m)}$ has been eliminated in the succeeding equations by using the first equation. We obtain an equivalent system by using these equations, since $(2 \cdot 11)$ is retained. Taking $2 m+p=k$, we obtain $h+p=h+k-2 m \leqslant 2 n$. The matrix of coefficients of $(2 \cdot 10)$ and $(2 \cdot 12)$ is

$$
\left(\begin{array}{ccccccccccccccc}
a_{0} & a_{1} & . . & a_{2 n-h-1} & a_{2 n-h} & . . & a_{2 n} & b_{0} & b_{1} & . . & b_{k-2 m-1} & b_{k-2 m} & b_{k-2 m+1} & . . & b_{k} \\
0 & 0 & . . & 0 & \gamma_{0} & . . & \gamma_{0 h} & 0 & 0 & . . & 0 & 1 & \delta_{01} & . . & \delta_{02} \\
0 & 0 & . . & \gamma_{0} & \gamma_{10} & . . & \gamma_{1 h} & 0 & 0 & . . & 1 & 0 & \delta_{11} & . . & \delta_{12 m} \\
\vdots & & & & & & & \vdots & & & & & & & \vdots \\
\Gamma_{0} & \tilde{\gamma}_{p 1} & . . & \tilde{\gamma}_{p p-1} & \gamma_{p 0} & . . & \gamma_{p h} & 1 & 0 & . . & 0 & 0 & \delta_{p 1} & . . & \delta_{p 2 m}
\end{array}\right),
$$

where $\Gamma_{0}$ (the term in the last row and first column) is defined as follows: if $h+$ $k<2(m+n)$, then $\Gamma_{0}=0$; if $h+k=2(m+n)$, then $\Gamma_{0}=\gamma_{0}$. If $h+k<2(m+n)$, then the terms $b_{0}, b_{1}, \ldots, b_{k-2 m}$ in the top row can be changed to zeros by subtracting multiples of the lower rows. The leading coefficient is unchanged in this process. If $h+k=2(n+m)$, then the term $\Gamma_{0}=\gamma_{0}$ is first converted to zero by subtracting $\gamma_{0} / a_{0} \times($ top row) from the last row. In this process, the 1 in the last row is converted to $\left(a_{0} d_{0}-b_{0} c_{0}\right) / a_{0} d_{0}$. By assumption $(\mathrm{A} 2 \cdot 4)$ this coefficient can have only isolated zeros for each value of $\lambda$. The term $b_{0}$ in the top row is now converted to zero by subtracting $a_{0} d_{0} b_{0} /\left(a_{0} d_{0}-b_{0} c_{0}\right) \times$ (bottom row) from the top row. In this process, singularities may be introduced into some coefficients in the top row. Finally, the (new) terms $b_{1}, b_{2}, \ldots, b_{k-2 m}$ in the top row are converted to zeros by subtracting multiples of the lower rows (above the last row). The new top row now corresponds to an equation of the form:

$$
a_{0}(x) y^{(2 n)}+\hat{a}_{1}(x, \lambda) y^{(2 n-1)}+\cdots+\hat{a}_{2 n}(x, \lambda) y+\hat{b}_{0}(x, \lambda) z^{(\hat{k})}+\cdots+\hat{b}_{\hat{k}}(x, \lambda) z=0,
$$

where $\hat{k} \leqslant 2 m-1$. If $m=0$, then $z$ and its derivatives do not appear in this equation. 


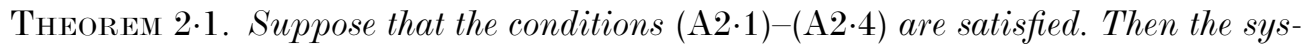
tem (2.3-2.6) can be transformed to an equivalent system of first order ODEs with separated boundary conditions. The system of ODEs has the form:

$$
\left(\begin{array}{l}
Y^{\prime} \\
Z^{\prime}
\end{array}\right)=G\left(\begin{array}{l}
Y \\
Z
\end{array}\right),
$$

where

$$
Y=\left(\begin{array}{c}
y_{0} \\
y_{1} \\
\vdots \\
y_{2 n-1}
\end{array}\right), \quad Z=\left(\begin{array}{c}
z_{0} \\
z_{1} \\
\vdots \\
z_{2 m-1}
\end{array}\right),
$$

and $G$ is a $2(m+n) \times 2(m+n)$ matrix whose coefficients are functions of $(x, \lambda)$ which may have singularities. If $k<2 m$, or if $m>0$ and $h+k<2(m+n)$, then these coefficients are non-singular. The boundary conditions corresponding to $(2 \cdot 5)$ and $(2 \cdot 6)$ are:

$$
\begin{array}{ll}
\sum_{\beta=0}^{2 n-1} p_{\alpha \beta} y_{\beta}(a)=0=\sum_{\beta=0}^{2 n-1} q_{\alpha \beta} y_{\beta}(b) & (1 \leqslant \alpha \leqslant n), \\
\sum_{\nu=0}^{2 m-1} r_{\mu \nu} z_{\nu}(a)=0=\sum_{\nu=0}^{2 m-1} s_{\mu \nu} z_{\nu}(b) & (1 \leqslant \mu \leqslant m) .
\end{array}
$$

If $m=0$, then $Z$ does not appear in the ODEs or the boundary conditions. However, in this case, the system must be supplemented by an equation of the form $z=f(Y)$.

Proof. By Lemma 2.1, we may assume that either $m=0$ or $k<2 m$. Set $Y=\left(\begin{array}{lll}y_{0} & y_{1} \cdots y_{2 n-1}\end{array}\right)^{T}=\left(\begin{array}{lll}y & y^{\prime} \cdots y^{(2 n-1)}\end{array}\right)^{T}$, and if $m>0$, set $Z=\left(z_{0} z_{1} \cdots z_{2 m-1}\right)^{T}=$ $\left(z z^{\prime} \cdots z^{(2 m-1)}\right)^{T}$. Then

$$
\begin{gathered}
y_{j}^{\prime}=y_{j+1} \quad(\text { for } 0 \leqslant j \leqslant 2 n-2), \\
y_{2 n-1}^{\prime}=a_{0}(x)^{-1}\left(a_{1} y_{2 m-1}+\cdots+a_{2 m} y_{0}+b_{0} z_{k}+\cdots b_{k} z_{0}\right), \\
z_{j}^{\prime}=z_{j+1} \quad(\text { for } 0 \leqslant j \leqslant 2 m-2), \\
z_{2 m-1}^{\prime}=d_{0}(x)^{-1}\left(c_{0} y_{k}+\cdots+c_{k} y_{0}+d_{1} z_{2 m-1}+\cdots d_{2 m} z_{0}\right) .
\end{gathered}
$$

If $m=0$, then $Z$ does not appear in the above equations.

The Ekman boundary layer

The analysis of stability for the Ekman boundary layer leads to the block operator problem $(1 \cdot 1)-(1 \cdot 2)$. In this case, $2 n=4,2 m=2$ and $h=k=1$. Thus we are in the case where $h+k<2(m+n)$. Following the procedure of Theorem $2 \cdot 1$, we can transform (1-1) to an equivalent system of first order ODEs. Set

$$
u=\left(\begin{array}{l}
u_{1} \\
u_{2} \\
u_{3} \\
u_{4}
\end{array}\right)=\left(\begin{array}{c}
y \\
y^{\prime} \\
y^{\prime \prime} \\
y^{\prime \prime \prime}
\end{array}\right), \quad v=\left(\begin{array}{c}
v_{1} \\
v_{2}
\end{array}\right)=\left(\begin{array}{c}
z \\
z^{\prime}
\end{array}\right) .
$$

Then $(1 \cdot 1)$ is equivalent to

$$
\left(\begin{array}{l}
u^{\prime} \\
v^{\prime}
\end{array}\right)=G\left(\begin{array}{l}
u \\
v
\end{array}\right),
$$


where

$$
G=\left(\begin{array}{cccccc}
0 & 1 & 0 & 0 & 0 & 0 \\
0 & 0 & 1 & 0 & 0 & 0 \\
0 & 0 & 0 & 1 & 0 & 0 \\
a_{41} & 0 & a_{43} & 0 & 0 & -2 \\
0 & 0 & 0 & 0 & 0 & 1 \\
a_{61} & 2 & 0 & 0 & a_{65} & 0
\end{array}\right),
$$

with

$$
\begin{aligned}
& a_{41}=-\left(\alpha^{4}+i \alpha^{3} R V+i \alpha R V^{\prime \prime}-\alpha^{2} \lambda\right), \\
& a_{43}=2 \alpha^{2}+i \alpha R V-\lambda, \\
& a_{61}=i \alpha R U^{\prime}, \\
& a_{65}=\alpha^{2}+i \alpha R V-\lambda .
\end{aligned}
$$

The boundary conditions are:

$$
u_{1}(0)=u_{2}(0)=v_{1}(0)=0,
$$

and

$$
u \in\left(L^{2}[0, \infty)\right)^{4}, \quad v \in\left(L^{2}[0, \infty)\right)^{2} .
$$

\section{The essential spectrum of the Ekman problem}

Throughout this section, we make extensive use of assumptions (A1·1-A1·3).

Let the matrix operator on the left-hand side of $(1 \cdot 1)$ be denoted by $M$, and on the right-hand side by $N$. Thus $(1 \cdot 1)$ has the form

$$
M \zeta=\lambda N \zeta, \text { where } \zeta=(y, z)^{T} .
$$

We are also interested in the operator

$$
L=M-\lambda N .
$$

The domains of the operators $L, M$ and $N$ will now be defined. Let $X_{1}=H^{2}[0, \infty) \cap$ $H_{0}^{1}[0, \infty)$ (with $H^{2}$-norm), $X_{2}=Y_{1}=Y_{2}=L^{2}[0, \infty)$ and $X=X_{1} \times X_{2}, Y=Y_{1} \times Y_{2}$. Let $D_{1}=H^{4}[0, \infty) \cap H_{0}^{2}[0, \infty)$ (with $H^{2}$-norm), and $D_{2}=H^{2}[0, \infty) \cap H_{0}^{1}[0, \infty)$ (with $L^{2}$-norm).

Define the domains: $\mathcal{D}(N)=X, \mathcal{D}(M)=D_{1} \times D_{2} \subset X, \mathrm{D}(\mathrm{L})=\mathrm{D}(\mathrm{M})$.

Then $N: X \rightarrow Y, M: \mathcal{D}(M) \subset X \rightarrow Y, L: \mathcal{D}(L) \subset X \rightarrow Y$.

The construction of the operators used here closely follows that to be found for the Orr-Sommerfeld case in Langer and Tretter [18] and for a more general abstract case in Shkalikov [24].

We shall say that $\lambda$ is an eigenvalue for $(M, N)$ if the operator $L=M-\lambda N$ is not injective.

As indicated in [11], several different definitions of essential spectrum have appeared in the literature. The definition we shall use here is:

$$
\begin{gathered}
\sigma_{e s s}(T)=\{\lambda \in \mathbb{C}: T-\lambda I \text { is not Fredholm }\}, \\
\sigma_{e s s}(M, N)=\{\lambda \in \mathbb{C}: M-\lambda N \text { is not Fredholm }\} .
\end{gathered}
$$


$N$ is bounded because the differential operator $-D^{2}+\alpha^{2}$ is a bounded operator from $H^{2}[0, \infty)$ to $L^{2}[0, \infty)$. It is also straightforward to show that $N$ is injective, with bounded inverse. Therefore (see, e.g., [11, theorem 3.16]) the operator $L=M-\lambda N$ is Fredholm if and only if $N^{-1} L=N^{-1} M-\lambda I$ is Fredholm (and the two operators have the same nullity, deficiency and index). Thus

$$
\sigma_{e s s}(M, N)=\sigma_{e s s}\left(N^{-1} M\right) .
$$

Another useful characterization is:

$$
\sigma_{\text {ess }}(M, N)=\left\{\lambda \in \mathbb{C}: 0 \in \sigma_{\text {ess }}(M-\lambda N)\right\} .
$$

Our aim is to calculate $\sigma_{e s s}(M, N)$. We shall begin by showing:

Theorem $3 \cdot 1$. The operators $L$ and $M$ are closed.

Proof. Since $N: X \rightarrow Y$ is an isomorphism of Hilbert spaces, $L=M-\lambda N$ is closed if $M$ is closed. To show that $M$ is closed, let

$$
\begin{gathered}
P(y, z)=\left(-D^{2}+\alpha^{2}\right)^{2} y+i \alpha R V\left(-D^{2}+\alpha^{2}\right) y+i \alpha R V^{\prime \prime} y+2 z^{\prime}, \\
Q(y, z)=\left(-D^{2}+\alpha^{2}\right) z+i \alpha R V z+2 y^{\prime}+i \alpha R U^{\prime} y .
\end{gathered}
$$

Then

$$
M\left(\begin{array}{l}
y \\
z
\end{array}\right)=\left(\begin{array}{l}
P(y, z) \\
Q(y, z)
\end{array}\right) .
$$

To show that the graph of $M$ is closed, suppose there is a sequence $\left(y_{n}, z_{n}\right)^{T} \in \mathcal{D}(M)$, such that $\left(y_{n}, z_{n}\right)^{T} \rightarrow\left(y_{0}, z_{0}\right)^{T}$ in $X$, and $\left(P\left(y_{n}, z_{n}\right), Q\left(y_{n}, z_{n}\right)\right)^{T} \rightarrow\left(P_{0}, Q_{0}\right)^{T}$ in $Y$. Hence $y_{n} \rightarrow y_{0}$ in $H^{2}$-norm, and $z_{n} \rightarrow z_{0}, P\left(y_{n}, z_{n}\right) \rightarrow P_{0}, Q\left(y_{n}, z_{n}\right) \rightarrow Q_{0}$ in $L^{2}$ norm. We must show that $P\left(y_{0}, z_{0}\right)=P_{0}$ and $Q\left(y_{0}, z_{0}\right)=Q_{0}$. Since $Q\left(y_{n}, z_{n}\right) \rightarrow Q_{0}$ in $L^{2}$ norm, it follows that

$$
\begin{aligned}
\lim _{n \rightarrow \infty}\left(-D^{2}+\alpha^{2}\right) z_{n} & =\lim _{n \rightarrow \infty}\left(Q\left(y_{n}, z_{n}\right)-2 y_{n}^{\prime}-i \alpha R U^{\prime} y_{n}-i \alpha R V z_{n}\right) \\
& =Q_{0}-2 y_{0}^{\prime}-i \alpha R U^{\prime} y_{0}-i \alpha R V z_{0}
\end{aligned}
$$

in $L^{2}$-norm, because hypotheses $(\mathrm{A} 1 \cdot 1-\mathrm{A} 1 \cdot 3)$ imply that $U^{\prime}$ and $V$ are bounded. But the operator $-D^{2}+\alpha^{2}$ with domain $H^{2}[0, \infty) \cap H_{0}^{1}[0, \infty)$ is closed (cf. [11, theorem XI.9.5]). Therefore $\lim _{n \rightarrow \infty}\left(-D^{2}+\alpha^{2}\right) z_{n}=\left(-D^{2}+\alpha^{2}\right) z_{0}$, and this implies that $Q\left(y_{0}, z_{0}\right)=Q_{0}$. Thus $z_{n} \rightarrow z_{0}$ in $H^{2}$-norm, therefore $z_{n}^{\prime} \rightarrow z_{0}^{\prime}$ in $L^{2}$-norm. Furthermore,

$$
\begin{aligned}
\lim _{n \rightarrow \infty}\left(-D^{2}+\alpha^{2}\right)^{2} y_{n} & =\lim _{n \rightarrow \infty}\left(P\left(y_{n}, z_{n}\right)-i \alpha R V\left(-D^{2}+\alpha^{2}\right) y_{n}-i \alpha R V^{\prime \prime} y_{n}-2 z_{n}^{\prime}\right) \\
& =P_{0}-i \alpha R V\left(-D^{2}+\alpha^{2}\right) y_{0}-i \alpha R V^{\prime \prime} y_{0}-2 z_{0}^{\prime}
\end{aligned}
$$

in $L^{2}$-norm. But [11, theorem IX.9.5] implies that the operator $\left(-D^{2}+\alpha^{2}\right)^{2}$ with domain $H^{4}[0, \infty) \cap H_{0}^{2}[0, \infty)$ is closed. Therefore $\lim _{n \rightarrow \infty}\left(-D^{2}+\alpha^{2}\right)^{2} y_{n}=\left(-D^{2}+\right.$ $\left.\alpha^{2}\right)^{2} y_{0}$, and so $P\left(y_{0}, z_{0}\right)=P_{0}$.

Define perturbations of $M$ and $L$ as follows. Let

$$
M_{0}=\left(\begin{array}{cc}
\left(-D^{2}+\alpha^{2}\right)^{2} & 2 D \\
2 D & -D^{2}+\alpha^{2}
\end{array}\right), \quad \mathcal{D}\left(M_{0}\right)=\mathcal{D}(M),
$$




$$
\begin{gathered}
K=\left(\begin{array}{cc}
i \alpha R V\left(-D^{2}+\alpha^{2}\right)+i \alpha R V^{\prime \prime} & 0 \\
i \alpha R U^{\prime} & i \alpha R V
\end{array}\right), \quad \mathcal{D}(K)=\mathcal{D}(M), \\
L_{0}=M_{0}-\lambda N, \quad \mathcal{D}\left(L_{0}\right)=\mathcal{D}(L)=\mathcal{D}(M) .
\end{gathered}
$$

Then $M=M_{0}+K$ and $L=L_{0}+K$.

Theorem $3 \cdot 2$. $K$ is $L_{0}$-compact.

Proof. Suppose there is a sequence $\left(y_{n}, z_{n}\right)^{T} \in \mathcal{D}\left(L_{0}\right)$ such that

$$
\left\|\left(y_{n}, z_{n}\right)^{T}\right\|_{L_{0}}^{2}:=\left\|\left(y_{n}, z_{n}\right)^{T}\right\|_{X}^{2}+\left\|L_{0}\left(y_{n}, z_{n}\right)^{T}\right\|_{Y}^{2} \leqslant C
$$

for some constant $C>0$. We must show that there is a subsequence (which we shall simply denote by $\left(y_{n}, z_{n}\right)^{T}$ again) such that $K\left(y_{n}, z_{n}\right)^{T}$ converges in $Y$. We shall use the notations: $H^{2}=H^{2}[0, \infty), L^{2}=L^{2}[0, \infty)$ and $\|u\|_{2}=\|u\|_{H^{2}},\|u\|=\|u\|_{L^{2}}$. Then $\left\|\left(y_{n}, z_{n}\right)^{T}\right\|_{X}^{2}=\left\|y_{n}\right\|_{2}^{2}+\left\|z_{n}\right\|^{2}$. Therefore $y_{n}$ is bounded in $H^{2}$ and $z_{n}$ is bounded in $L^{2}$.

Let $P_{1}: Y \rightarrow Y_{1}$ and $P_{2}: Y \rightarrow Y_{2}$ be the orthogonal projections, and let

$$
L_{1}=P_{1} L_{0}, \quad L_{2}=P_{2} L_{0}, \quad K_{1}=P_{1} K, \quad K_{2}=P_{2} K .
$$

Since $\|y\|_{Y}^{2}=\left\|P_{1} y\right\|^{2}+\left\|P_{2} y\right\|^{2}$ for $y \in Y$, we see that $L_{1}\left(y_{n}, z_{n}\right)^{T}$ and $L_{2}\left(y_{n}, z_{n}\right)^{T}$ are bounded in $L^{2}$, and we must show that there is a subsequence such that $K_{1}\left(y_{n}, z_{n}\right)^{T}$ and $K_{2}\left(y_{n}, z_{n}\right)^{T}$ converge in $L^{2}$.

A calculation shows that:

$$
\begin{gathered}
L_{1}\left(y_{n}, z_{n}\right)^{T}=\left(-D^{2}+\alpha^{2}\right)^{2} y_{n}-\lambda\left(-D^{2}+\alpha^{2}\right) y_{n}+2 z_{n}^{\prime}, \\
L_{2}\left(y_{n}, z_{n}\right)^{T}=2 y_{n}^{\prime}+\left(-D^{2}+\alpha^{2}\right) z_{n}-\lambda z_{n} \\
K_{1}\left(y_{n}, z_{n}\right)^{T}=i \alpha R V\left(-D^{2}+\alpha^{2}\right) y_{n}+i \alpha R V^{\prime \prime} y_{n} \\
K_{2}\left(y_{n}, z_{n}\right)^{T}=i \alpha R U^{\prime} y_{n}+i \alpha R V z_{n} .
\end{gathered}
$$

Since $y_{n}$ is bounded in $H^{2}, y_{n}^{\prime}$ is bounded in $L^{2}$. Now $y_{n}^{\prime}, z_{n}$ and $L_{2}\left(y_{n}, z_{n}\right)^{T}$ bounded in $L^{2}$ imply that $\left(-D^{2}+\alpha^{2}\right) z_{n}$ is bounded in $L^{2}$. Let $A(z)=\left(-D^{2}+\alpha^{2}\right) z$, with $\mathcal{D}(A)=D_{2}$, and $B(z)=i \alpha R V z$, with $\mathcal{D}(B)=\left\{z: z, B(z) \in L^{2}\right\}$. Since $\lim _{x \rightarrow \infty} V(x)=0$ and $B$ has lower order that $A,[\mathbf{1 1}$, theorem IX·8.2] implies that $B$ is $A$-compact. Therefore there is a subsequence so that $i \alpha R V z_{n}$ converges in $L^{2}$. Similarly, since $\left(-D^{2}+\alpha^{2}\right) y_{n}$ is bounded in $L^{2}$, there is a subsequence such that $i \alpha R U^{\prime} y_{n}$ converges in $L^{2}$. This shows that there is a subsequence such that $K_{2}\left(y_{n}, z_{n}\right)^{T}$ converges in $L^{2}$.

Now we must show that there is a subsequence so that $K_{1}\left(y_{n}, z_{n}\right)^{T}$ converges in $L^{2}$. Since $z_{n}$ and $\left(-D^{2}+\alpha^{2}\right) z_{n}$ are bounded in $L^{2}, z_{n}$ is bounded in $H^{2}$. This implies that $z_{n}^{\prime}$ is bounded in $L^{2}$. This together with the boundedness of $L_{1}\left(y_{n}, z_{n}\right)^{T}$ in $L^{2}$ implies that $\left(-D^{2}+\alpha^{2}\right)^{2} y_{n}-\lambda\left(-D^{2}+\alpha^{2}\right) y_{n}$ is bounded in $L^{2}$. [11, theorem IX $\cdot 8 \cdot 2$ ] now implies the existence of a subsequence so that $K_{1}\left(y_{n}, z_{n}\right)^{T}$ converges in $L^{2}$.

Corollary 3·1. $\sigma_{\text {ess }}(M, N)=\sigma_{\text {ess }}\left(M_{0}, N\right)$.

Proof. Since $K$ is $L_{0}$-compact, and $L=L_{0}+K$, it follows that $\sigma_{e s s}(L)=\sigma_{e s s}\left(L_{0}\right)$. (cf. [11, theorem XI·2.1]). Furthermore,

$$
\sigma_{\text {ess }}(M, N)=\left\{\lambda \in \mathbb{C}: 0 \in \sigma_{\text {ess }}(L)\right\} \text { and } \sigma_{\text {ess }}\left(M_{0}, N\right)=\left\{\lambda \in \mathbb{C}: 0 \in \sigma_{\text {ess }}\left(L_{0}\right)\right\} .
$$

Therefore $\sigma_{e s s}(M, N)=\sigma_{e s s}\left(M_{0}, N\right)$. 
Our task is now to calculate $\sigma_{\text {ess }}\left(M_{0}, N\right)$. For this purpose, we transform the equation

$$
L_{0}\left(\begin{array}{l}
y \\
z
\end{array}\right)=\left(\begin{array}{l}
f \\
g
\end{array}\right)
$$

to an equivalent system of first order ODEs, as in $(2 \cdot 13)-(2 \cdot 15)$. We set

$$
u=\left(\begin{array}{l}
u_{1} \\
u_{2} \\
u_{3} \\
u_{4}
\end{array}\right)=\left(\begin{array}{c}
y \\
y^{\prime} \\
y^{\prime \prime} \\
y^{\prime \prime \prime}
\end{array}\right), \quad v=\left(\begin{array}{l}
v_{1} \\
v_{2}
\end{array}\right)=\left(\begin{array}{c}
z \\
z^{\prime}
\end{array}\right)
$$

and obtain

$$
\left(\begin{array}{l}
u^{\prime} \\
v^{\prime}
\end{array}\right)-A\left(\begin{array}{l}
u \\
v
\end{array}\right)=h
$$

where

$$
A=\left(\begin{array}{cccccc}
0 & 1 & 0 & 0 & 0 & 0 \\
0 & 0 & 1 & 0 & 0 & 0 \\
0 & 0 & 0 & 1 & 0 & 0 \\
a_{41} & 0 & a_{43} & 0 & 0 & -2 \\
0 & 0 & 0 & 0 & 0 & 1 \\
0 & 2 & 0 & 0 & a_{65} & 0
\end{array}\right), \quad h=\left(\begin{array}{c}
0 \\
0 \\
0 \\
f \\
0 \\
-g
\end{array}\right)
$$

with

$$
\begin{aligned}
& a_{41}=-\alpha^{4}+\alpha^{2} \lambda, \\
& a_{43}=2 \alpha^{2}-\lambda, \\
& a_{65}=\alpha^{2}-\lambda .
\end{aligned}
$$

The boundary conditions are:

$$
u_{1}(0)=u_{2}(0)=v_{1}(0)=0
$$

and

$$
u \in\left(L^{2}[0, \infty)\right)^{4}, \quad v \in\left(L^{2}[0, \infty)\right)^{2} .
$$

The characteristic polynomial of $A$ is

$$
\begin{aligned}
p(\mu)=p(\mu ; \lambda) & =\operatorname{det}(\mu I-A)=\left(\mu^{2}-\alpha^{2}+\lambda\right)^{2}\left(\mu^{2}-\alpha^{2}\right)+4 \mu^{2} \\
& =\mu^{6}+\left(2 \lambda-3 \alpha^{2}\right) \mu^{4}+\left(\lambda^{2}-4 \alpha^{2} \lambda+3 \alpha^{4}+4\right) \mu^{2}-\alpha^{2}\left(\lambda-\alpha^{2}\right)^{2} .
\end{aligned}
$$

The roots of $p(\mu)$ are the values of an algebraic function $\mu=\mu(\lambda)$. They live on a Riemann surface which is a 6 -sheeted branched covering of $\mathbb{C}$. There are finitely many points $\lambda \in \mathbb{C}$ over which the Riemann surface is branched. These are the roots of the discriminant of $p(\mu)$, and will be called the branch points. They are the values of $\lambda$ such that $p(\mu)$ has multiple roots. Thus, with the exception of finitely many points $\lambda, p(\mu)$ has 6 different roots. Since $p(\mu)$ is an even polynomial, the roots occur in \pm pairs. The roots $+\mu$ and $-\mu$ are different, except for the root $\mu=0$, which occurs for $\lambda=\alpha^{2}$. When no root is purely imaginary, they can be ordered: $\mu_{1}, \mu_{2}, \mu_{3}, \mu_{4}, \mu_{5}, \mu_{6}$, such that $\operatorname{Re}\left(\mu_{k}\right)<0$ for $1 \leqslant k \leqslant 3$ and $\operatorname{Re}\left(\mu_{k}\right)>0$ for $4 \leqslant k \leqslant 6$. This is important because the function $e^{\mu_{k} x} \in L^{2}[0, \infty)$ for $1 \leqslant k \leqslant 3$, and $e^{\mu_{k} x} \notin L^{2}[0, \infty)$ for $4 \leqslant k \leqslant 6$. 
We will show that $\sigma_{e s s}\left(M_{0}, N\right)=\mathbb{S}\left(M_{0}, N\right)$, where

$$
\mathbb{S}\left(M_{0}, N\right)=\{\lambda \in \mathbb{C}: p(\mu) \text { has a purely imaginary root }\} .
$$

We shall now identify this set explicitly.

Theorem $3 \cdot 3$. The following are equivalent.

(1) $\lambda \in \mathbb{S}\left(M_{0}, N\right)$.

(2) $\left(\lambda-\alpha^{2}-\xi^{2}\right)^{2}\left(\alpha^{2}+\xi^{2}\right)+4 \xi^{2}=0$ for some $\xi \in \mathbb{R}$.

(This equation is satisfied if and only if $\mu(\lambda)=i \xi$ is a root of $p(\mu ; \lambda)$.)

(3) $\lambda=\nu+i \eta$, where $\eta^{2}=4\left(1-\alpha^{2} / \nu\right), \nu \geqslant \alpha^{2}$.

Proof. (1) and (2) are equivalent, since (2) is is the result of setting $\mu=i \xi$ in the characteristic equation of $A:\left(\mu^{2}-\alpha^{2}+\lambda\right)^{2}\left(\mu^{2}-\alpha^{2}\right)+4 \mu^{2}=0$.

Solving for $\lambda$ in (2), we obtain:

$$
\lambda=\left(\alpha^{2}+\xi^{2}\right) \pm \frac{2 i \xi}{\sqrt{\alpha^{2}+\xi^{2}}} .
$$

Thus

$$
\nu=\alpha^{2}+\xi^{2} \quad \text { and } \quad \eta= \pm \frac{2 \sqrt{\nu-\alpha^{2}}}{\sqrt{\nu}}= \pm 2 \sqrt{1-\frac{\alpha^{2}}{\nu}} .
$$

This shows that (2) is equivalent to (3).

Recall the following definition of "singular sequence" for a linear map $T: \mathcal{D}(T) \subset$ $X \rightarrow X$. (cf. [11, definition IX.1.2].) A sequence $\left\{x_{n}: n \in \mathbb{N}\right\} \subset \mathcal{D}(T)$ is called a singular sequence for $T-\lambda I$ if the following conditions are satisfied:

(1) it has no convergent subsequence;

(2) $\left\|x_{n}\right\|_{X}=1$ for $n \in \mathbb{N}$;

(3) $\lim _{n \rightarrow \infty}(T-\lambda I) x_{n}=0$.

Condition (2) may be relaxed to:

(2') $0<a<\left\|x_{n}\right\|_{X}<b$ for $n \geqslant N_{0}$.

Theorem IX.1.3 in [11] implies that if $T$ is a closed, densely defined operator such that $T-\lambda I$ has a singular sequence, then $\lambda \in \sigma_{e s s}(T)$.

Similarly, we shall define "singular sequence" for $M_{0}-\lambda N$ as a sequence $\left\{x_{n}: n \in\right.$ $\mathbb{N}\}$ satisfying (1) and (2') above, and

(3') $\lim _{n \rightarrow \infty}\left(M_{0}-\lambda N\right) x_{n}=0$.

Clearly, a singular sequence for $M_{0}-\lambda N$ is also a singular sequence for $N^{-1} M_{0}-\lambda I$. Since $\sigma_{e s s}\left(M_{0}, N\right)=\sigma_{e s s}\left(N^{-1} M_{0}\right)$, we see that if $M_{0}-\lambda N$ has a singular sequence, then $\lambda \in \sigma_{e s s}\left(M_{0}, N\right)$.

Theorem 3.4. $\mathbb{S}\left(M_{0}, N\right) \subset \sigma_{\text {ess }}(M, N)$.

Proof. Using an idea from the proof of [11, theorem IX.7.3], we shall construct a singular sequence for $M_{0}-\lambda N$, when $\lambda \in \mathbb{S}\left(M_{0}, N\right)$. Note that $\mu(\lambda)=0$ is a root of $p(\mu ; \lambda)$ only when $\lambda=\alpha^{2}$. We shall first consider the case $\lambda \neq \alpha^{2}$.

Let $\eta \in C^{\infty}(-\infty, \infty)$ be a function satisfying the following conditions: $\eta(x)=1$, for $|x| \leqslant 1 / 2 ; \eta(x)=0$, for $|x| \geqslant 1$; and $0 \leqslant \eta(x) \leqslant 1$ on $(-\infty, \infty)$. Let 
$I_{n}=\left\{x:\left|x-x_{n}\right| \leqslant \ell_{n}\right\}(n \in \mathbb{N})$ be mutually disjoint intervals in $(0, \infty)$, with $\lim _{n \rightarrow \infty} \ell_{n}=\infty$. Suppose that $\lambda \in \mathbb{S}\left(M_{0}, N\right)$. Then there is a root $\mu(\lambda)=i \xi$ of $p(\mu, \lambda)$, where $\xi \in \mathbb{R} ; \xi \neq 0$, since we have assumed that $\lambda \neq \alpha^{2}$. We define:

$$
\begin{aligned}
& y_{n}(x)=\ell_{n}^{-1 / 2}\left(-D^{2}+\alpha^{2}-\lambda\right)\left(\eta\left[\left(x-x_{n}\right) \ell_{n}^{-1}\right] \exp \left[i \xi\left(x-x_{n}\right)\right]\right), \\
& z_{n}(x)=-2 \ell^{-1 / 2} D\left(\eta\left[\left(x-x_{n}\right) \ell_{n}^{-1}\right] \exp \left[i \xi\left(x-x_{n}\right)\right]\right), \\
& \zeta_{n}(x)=\left(y_{n}(x), z_{n}(x)\right)^{T}
\end{aligned}
$$

where $D=d / d x$. We claim that $\left\{\zeta_{n}: n \in \mathbb{N}\right\}$ is a singular sequence for $M_{0}-\lambda N$.

Since $\operatorname{Supp}\left(\zeta_{n}\right) \subset I_{n}$, and the intervals $I_{n}$ are disjoint, the functions $\zeta_{n}$ are orthogonal to each other. Therefore condition (2') $\left(0<a<\left\|\zeta_{n}\right\|_{X}<b\right.$, for $\left.n \geqslant N_{0}\right)$ implies (1) (there is no convergent subsequence). It remains to prove $\left(2^{\prime}\right)$ and $\left(3^{\prime}\right)$.

Proof of (2'). We start with the observation

$$
\left\|\zeta_{n}\right\|_{X}^{2}=\left\|y_{n}\right\|_{2}^{2}+\left\|z_{n}\right\|^{2}=\left\|D^{2} y_{n}\right\|^{2}+\left\|D y_{n}\right\|^{2}+\left\|y_{n}\right\|^{2}+\left\|z_{n}\right\|^{2} .
$$

Let

$$
w_{n}=\ell_{n}^{-1 / 2} g(D)\left(\eta\left[\left(x-x_{n}\right) \ell_{n}^{-1}\right] \exp \left[i \xi\left(x-x_{n}\right)\right]\right),
$$

where $g(t)=\sum_{j=0}^{k} a_{j} t^{j},\left(a_{j} \in \mathbb{C}, 0 \leqslant j \leqslant k\right)$. There are constants $b_{j}=b_{j}(\xi)$ so that

$$
\begin{gathered}
w_{n}(x)=\ell_{n}^{-1 / 2}\left(\sum_{j=1}^{k} b_{j} \ell_{n}^{-j} \eta^{(j)}\left[\left(x-x_{n}\right) \ell_{n}^{-1}\right]+g(i \xi) \eta\left[\left(x-x_{n}\right) \ell_{n}^{-1}\right]\right) \exp \left[\left(i \xi\left(x-x_{n}\right)\right],\right. \\
\int_{\left|x-x_{n}\right| \leqslant \ell_{n} / 2}\left|w_{n}\right|^{2} d x \leqslant\left\|w_{n}\right\|^{2}=\int_{\left|x-x_{n}\right| \leqslant \ell_{n}}\left|w_{n}(x)\right|^{2} d x \\
\int_{\left|x-x_{n}\right| \leqslant \ell_{n}}\left|w_{n}(x)\right|^{2} d x=\int_{|x| \leqslant 1}\left|\sum_{j=1}^{k} b_{j} \ell_{n}^{-j} \eta^{(j)}(x)+g(i \xi) \eta(x)\right|^{2} d x
\end{gathered}
$$

Given $\epsilon>0$, there exists $N=N(g)$, such that for $n \geqslant N$, (and for all $x$ )

$$
\left|\sum_{j=1}^{k} b_{j} \ell_{n}^{-j} \eta^{(j)}(x)\right| \leqslant \epsilon .
$$

Using the inequality $|p+q|^{2} \leqslant 2\left(|p|^{2}+|q|^{2}\right)$ (for $p, q \in \mathbb{C}$ ), we obtain (for $n \geqslant N$ )

$$
\left\|w_{n}\right\|^{2} \leqslant 2 \int_{-1}^{1}\left(\epsilon^{2}+|g(i \xi)|^{2} \eta(x)^{2}\right) d x
$$

and since $0 \leqslant \eta(x) \leqslant 1$,

$$
\left\|w_{n}\right\|^{2} \leqslant 4\left(\epsilon^{2}+|g(i \xi)|^{2}\right), \text { for } n \geqslant N .
$$

Using the inequality $|p+q|^{2} \geqslant 1 / 2|p|^{2}-|q|^{2}$ (from $2|p q| \leqslant 1 / 2|p|^{2}+2|q|^{2}$ ), we find

$$
\left\|w_{n}\right\|^{2} \geqslant \int_{-1 / 2}^{1 / 2}\left(1 / 2|g(i \xi)|^{2} \eta(x)^{2}-\epsilon^{2}\right) d x=1 / 2|g(i \xi)|^{2}-\epsilon^{2} .
$$

Thus we have

$$
1 / 2|g(i \xi)|^{2}-\epsilon^{2} \leqslant\left\|w_{n}\right\|^{2} \leqslant 4\left(\epsilon^{2}+|g(i \xi)|^{2}\right), \text { for } n \geqslant N(g) .
$$


Applying (3.6) to $y_{n}, D y_{n}, D^{2} y_{n}$ and $z_{n}$, we obtain (for any given $\epsilon>0$ and sufficiently large $n)$ :

$$
\begin{aligned}
1 / 2\left|\xi^{2}+\alpha^{2}-\lambda\right|^{2}-\epsilon^{2} & \leqslant\left\|y_{n}\right\|^{2} \leqslant 4\left(\left|\xi^{2}+\alpha^{2}-\lambda\right|^{2}+\epsilon^{2}\right) \\
1 / 2 \xi^{2}\left|\xi^{2}+\alpha^{2}-\lambda\right|^{2}-\epsilon^{2} & \leqslant\left\|D y_{n}\right\|^{2} \leqslant 4\left(\xi^{2}\left|\xi^{2}+\alpha^{2}-\lambda\right|^{2}+\epsilon^{2}\right) \\
1 / 2 \xi^{4}\left|\xi^{2}+\alpha^{2}-\lambda\right|^{2}-\epsilon^{2} & \leqslant\left\|D^{2} y_{n}\right\|^{2} \leqslant 4\left(\xi^{4}\left|\xi^{2}+\alpha^{2}-\lambda\right|^{2}+\epsilon^{2}\right) .
\end{aligned}
$$

This proves condition (2').

Proof of (3'). We start with the equation

where

$$
\left(M_{0}-\lambda N\right)\left(\begin{array}{l}
y \\
z
\end{array}\right)=\left(\begin{array}{l}
P(y, z) \\
Q(y, z)
\end{array}\right)
$$

$$
\begin{aligned}
& P(y, z)=\left(-D^{2}+\alpha^{2}\right)^{2} y-\lambda\left(-D^{2}+\alpha^{2}\right) y+2 D z, \\
& Q(y, z)=2 D y+\left(-D^{2}+\alpha^{2}\right) z-\lambda z .
\end{aligned}
$$

Applying this to $\zeta_{n}=\left(y_{n}, z_{n}\right)^{T}$, we obtain

$$
\begin{aligned}
P\left(y_{n}, z_{n}\right)= & \ell_{n}^{-1 / 2}\left\{\left[\left(-D^{2}+\alpha^{2}\right)^{2}-\lambda\left(-D^{2}+\alpha^{2}\right)\right]\left(-D^{2}+\alpha^{2}-\lambda\right)\right. \\
& \left.-4 D^{2}\right\} \eta\left[\left(x-x_{n}\right) \ell_{n}^{-1}\right] \exp \left[i \xi\left(x-x_{n}\right)\right] .
\end{aligned}
$$

By $(3 \cdot 5)$, for any $\epsilon>0$, there is an integer $N_{0}$ so that

$$
\left\|P\left(y_{n}, z_{n}\right)\right\|^{2} \leqslant 4\left(\epsilon^{2}+|g(i \xi)|^{2}\right), \text { for } n \geqslant N_{0},
$$

where

$$
\left.g(i \xi)=\left[\left(\xi^{2}+\alpha^{2}\right)^{2}-\lambda\left(\xi^{2}+\alpha^{2}\right)\right]\left(\xi^{2}+\alpha^{2}-\lambda\right)+4 \xi^{2}=\left(\xi^{2}+\alpha^{2}-\lambda\right)^{2}\right)\left(\xi^{2}+\alpha^{2}\right)+4 \xi^{2} .
$$

By Theorem $3 \cdot 3(2) \quad g(i \xi)=0$, and so $\left\|P\left(y_{n}, z_{n}\right)\right\| \leqslant 2 \epsilon$ for $n \geqslant N_{0}$. Therefore $\lim _{n \rightarrow \infty} P\left(y_{n}, z_{n}\right)=0$.

$$
\begin{aligned}
Q\left(y_{n}, z_{n}\right)= & \ell_{n}^{-1 / 2}\left[2 D\left(-D^{2}+\alpha^{2}-\lambda\right)-2\left(-D^{2}+\alpha^{2}\right) D\right. \\
& +2 \lambda D] \eta\left[\left(x-x_{n}\right) \ell_{n}^{-1}\right] \exp \left[i \xi\left(x-x_{n}\right)\right]=0 .
\end{aligned}
$$

This proves condition (3') when $\lambda \neq \alpha^{2}$.

We must still consider the case $\lambda=\alpha^{2}, \xi=0$. Define

$$
y_{n}(x)=\ell_{n}^{-1 / 2} \eta\left[\left(x-x_{n}\right) \ell_{n}^{-1}\right], z_{n}(x)=0, \quad \zeta_{n}(x)=\left(y_{n}(x), z_{n}(x)\right)^{T} .
$$

Then

$$
\begin{gathered}
\left\|\zeta_{n}\right\|_{X}^{2}=\left\|y_{n}\right\|^{2}+\left\|D y_{n}\right\|^{2}+\left\|D^{2} y_{n}\right\|^{2} \\
\int_{\left|x-x_{n}\right| \leqslant \ell_{n} / 2} \ell_{n}^{-1} \eta\left[\left(x-x_{n}\right) \ell_{n}^{-1}\right]^{2} d x \leqslant\left\|y_{n}\right\|^{2}=\int_{\left|x-x_{n}\right| \leqslant \ell_{n}} \ell_{n}^{-1} \eta\left[\left(x-x_{n}\right) \ell_{n}^{-1}\right]^{2} d x, \\
1=\int_{-1 / 2}^{1 / 2} \eta(x)^{2} d x \leqslant\left\|y_{n}\right\|^{2} \leqslant \int_{-1}^{1} \eta(x)^{2} d x \leqslant 2 .
\end{gathered}
$$

By $(3 \cdot 5)$, with $\xi=0$,

$$
\left\|D y_{n}\right\|^{2} \leqslant 4 \epsilon^{2}, \quad 0 \leqslant\left\|D^{2} y_{n}\right\|^{2} \leqslant 4 \epsilon^{2} .
$$

Therefore condition (2') is satisfied, and this implies condition (1). 
Using the fact that $\lambda=\alpha^{2}$, we have

$$
P\left(y_{n}, z_{n}\right)=\ell_{n}^{-1 / 2}\left[\left(-D^{2}+\alpha^{2}\right)^{2}-\alpha^{2}\left(-D^{2}+\alpha^{2}\right)\right] \eta\left[\left(x-x_{n}\right) \ell_{n}^{-1}\right] .
$$

By $(3 \cdot 5)$, for any $\epsilon>0$ there exists an integer $N_{1}$, such that for $n \geqslant N_{1}$

$$
\left\|P\left(y_{n}, z_{n}\right)\right\|^{2} \leqslant 4\left(\epsilon^{2}+|g(0)|^{2}\right)
$$

where $g(t)=\left(-t^{2}+\alpha^{2}\right)^{2}-\alpha^{2}\left(-t^{2}+\alpha^{2}\right)$. Thus $g(0)=0$, and

$$
\left\|P\left(y_{n}, z_{n}\right)\right\| \leqslant 2 \epsilon \text { for } n \geqslant N_{1} \text {. }
$$

We also have

$$
Q\left(y_{n}, z_{n}\right)=2 \ell_{n}^{-1 / 2} D \eta\left[\left(x-x_{n}\right) \ell_{n}^{-1}\right] .
$$

By $(3 \cdot 5)$ for $\epsilon>0$ there is an integer $N_{2}$ such that for $n \geqslant N_{2}$

$$
\left\|Q\left(y_{n}, z_{n}\right)\right\|^{2} \leqslant 4\left(\epsilon^{2}+|h(0)|^{2}\right),
$$

where $h(t)=2 t$. Thus $h(0)=0$ and $\left\|Q\left(y_{n}, z_{n}\right)\right\| \leqslant 2 \epsilon$ for $n \geqslant N_{1}$. Hence $\lim _{n \rightarrow \infty}\left(M_{0}-\right.$ $\left.\alpha^{2} N\right) \zeta_{n}=0$.

Lemma $3 \cdot 1$. Let $f, g, h \in L^{2}[0, \infty)$, and let

$$
\begin{aligned}
F(x)= & \int_{0}^{x} e^{\mu(x-t)} f(t) d t+a e^{\mu x} \\
G(x)= & \int_{0}^{x} e^{\mu(x-t)} g(t) d t+\int_{0}^{x} e^{\mu(x-t)}\left(\int_{0}^{t} e^{\mu(t-s)} f(s) d s\right) d t+a x e^{\mu x}+b e^{\mu x}, \\
H(x)= & \int_{0}^{x} e^{\mu(x-t)} h(t) d t+\int_{0}^{x} e^{\mu(x-t)}\left(\int_{0}^{t} e^{\mu(t-s)} g(s) d s\right) d t \\
& +\int_{0}^{x} e^{\mu(x-t)}\left[\int_{0}^{t} e^{\mu(t-s)}\left(\int_{0}^{s} e^{\mu(s-t)} f(r) d r\right) d s\right] d t+\frac{a}{2} x^{2} e^{\mu x}+b x e^{\mu x}+c e^{\mu x} .
\end{aligned}
$$

(1) If $\operatorname{Re}(\mu)<0$, then $F, G, H \in L^{2}[0, \infty)$ for any values of $a, b$, and $c$.

(2) If $\operatorname{Re}(\mu)>0$, then there are unique values of $a, b$, c such that $F, G, H \in L^{2}[0, \infty)$.

These values are:

$$
\begin{aligned}
& a=F(0)=-\int_{0}^{\infty} e^{-\mu t} f(t) d t \\
& b=G(0)=-\int_{0}^{\infty} e^{-\mu t} g(t) d t-\int_{0}^{\infty} e^{-\mu t} F(t) d t \\
& c=H(0)=-\int_{0}^{\infty} e^{-\mu t} h(t) d t-\int_{0}^{\infty} e^{-\mu t} G(t) d t .
\end{aligned}
$$

When $a, b$, and $c$ have these values, $F, G$ and $H$ have the following alternative formulas:

$$
\begin{aligned}
& F(x)=-\int_{x}^{\infty} e^{\mu(x-t)} f(t) d t \\
& G(x)=-\int_{x}^{\infty} e^{\mu(x-t)} g(t) d t-\int_{x}^{\infty} e^{\mu(x-t)} F(t) d t \\
& H(x)=-\int_{x}^{\infty} e^{\mu(x-t)} h(t) d t-\int_{x}^{\infty} e^{\mu(x-t)} G(t) d t .
\end{aligned}
$$


Proof. (1) Suppose $\operatorname{Re}(\mu)<0$. Note that if we prove that $F \in L^{2}[0, \infty)$ (for arbitrary $f \in L^{2}[0, \infty)$ ), then it follows that $G, H \in L^{2}[0, \infty)$. To show that $F \in L^{2}[0, \infty)$, extend $f$ to $(-\infty, \infty)$ by defining $f(x)=0$, for $x<0$, and consider the Fourier transform $\hat{f}(\omega)=\int_{-\infty}^{\infty} e^{-2 \pi i \omega t} f(t) d t$. Then

$$
\begin{aligned}
\int_{0}^{x} e^{\mu(x-t)} f(t) d t & =\int_{0}^{x} e^{\mu(x-t)}\left(\int_{-\infty}^{\infty} e^{2 \pi i \omega t} \hat{f}(\omega) d \omega\right) d t \\
& =\int_{-\infty}^{\infty} d \omega \int_{0}^{x} e^{\mu x} e^{(2 \pi i \omega-\mu) t} \hat{f}(\omega) d t \\
& =\int_{-\infty}^{\infty} e^{\mu x}(2 \pi i \omega-\mu)^{-1}\left[e^{(2 \pi i \omega-\mu) x}-1\right] \hat{f}(\omega) d \omega \\
& =\int_{-\infty}^{\infty} e^{2 \pi i \omega x}(2 \pi i \omega-\mu)^{-1} \hat{f}(\omega) d \omega-e^{\mu x} \int_{-\infty}^{\infty}(2 \pi i \omega-\mu)^{-1} \hat{f}(\omega) d \omega
\end{aligned}
$$

The first term on the last line $\in L^{2}[0, \infty)$ because it is the inverse Fourier transform of an $L^{2}$-function. The second term $\in L^{2}[0, \infty)$ because the exponential is an $L^{2}$ function, and the integral converges since it is the inner product of two $L^{2}$-functions. Changing the order of integration is justified by the definition of the Fourier transform on $L^{2}(-\infty, \infty)$ (as the $L^{2}$-limit of Fourier transforms on $L^{1}(-\infty, \infty)$ ).

(2) Suppose $\operatorname{Re}(\mu)>0$. Let $a=-\int_{0}^{\infty} e^{-\mu t)} f(t) d t$. Then

$$
\begin{aligned}
F(x) & =\int_{0}^{x} e^{\mu(x-t)} f(t) d t+a e^{\mu x} \\
& =\int_{0}^{x} e^{\mu(x-t)} f(t) d t-\int_{0}^{\infty} e^{\mu(x-t)} f(t) d t=-\int_{x}^{\infty} e^{\mu(x-t)} f(t) d t .
\end{aligned}
$$

Thus

$$
\begin{aligned}
F(x) & =-\int_{x}^{\infty} e^{\mu(x-t)} f(t) d t=-\int_{x}^{\infty} e^{\mu(x-t)}\left(\int_{-\infty}^{\infty} e^{2 \pi i \omega t} \hat{f}(\omega) d \omega\right) d t \\
& =-\int_{-\infty}^{\infty} d \omega \int_{x}^{\infty} e^{\mu x} e^{(2 \pi i \omega-\mu) t} \hat{f}(\omega) d t=-\int_{-\infty}^{\infty} e^{\mu x}(2 \pi i \omega-\mu)^{-1}\left[-e^{(2 \pi i \omega-\mu) x)}\right] \hat{f}(\omega) d \omega \\
& =\int_{-\infty}^{\infty} e^{2 \pi i \omega x}(2 \pi i \omega-\mu)^{-1} \hat{f}(\omega) d \omega,
\end{aligned}
$$

and so $F \in L^{2}[0, \infty)$.

To show that $G \in L^{2}[0, \infty)$, we note that since $\int_{0}^{t} e^{\mu(t-s)} f(s)=F(t)-a e^{\mu t}$, it follows that

$$
\int_{0}^{x} e^{\mu(x-t)}\left(\int_{0}^{t} e^{\mu(t-s)} f(s) d s\right) d t=\int_{0}^{x} e^{\mu(x-t)} F(t) d t-a x e^{\mu x}
$$

and

$$
G(x)=\int_{0}^{x} e^{\mu(x-t)} g(t) d t+\int_{0}^{x} e^{\mu(x-t)} F(t) d t+b e^{\mu x} .
$$

If we put

$$
b=-\int_{x}^{\infty} e^{\mu(x-t)} g(t) d t-\int_{x}^{\infty} e^{\mu(x-t)} F(t) d t,
$$


then

$$
G(x)=-\int_{0}^{\infty} e^{-\mu t} g(t) d t-\int_{0}^{\infty} e^{-\mu t} F(t) d t .
$$

Therefore $G \in L^{2}[0, \infty)$ by the same argument as for $F$.

We use a similar argument to show that $H \in L^{2}[0, \infty)$. Since

$$
\int_{0}^{t} e^{\mu(t-s)}\left(\int_{0}^{s} e^{\mu(s-r)} f(r) d r\right) d s=G(t)-\int_{0}^{t} e^{\mu(t-s)} g(s) d s-a t e^{\mu t}-b e^{\mu t},
$$

it follows that

$$
\begin{aligned}
& \int_{0}^{x} e^{\mu(x-t)}\left[\int_{0}^{t} e^{\mu(t-s)}\left(\int_{0}^{s} e^{\mu(s-r)} f(r) d r\right) d s\right] d t \\
& =\int_{0}^{x} e^{\mu(x-t)} G(t) d t-\int_{0}^{x} e^{\mu(x-t)}\left(\int_{0}^{t} e^{\mu(t-s)} g(s) d s\right) d t-\frac{a}{2} x^{2} e^{\mu x}-b x e^{\mu x}
\end{aligned}
$$

and

$$
H(x)=\int_{0}^{x} e^{\mu(x-t)} h(t) d t+\int_{0}^{x} e^{\mu(x-t)} G(t) d t+c e^{\mu x} .
$$

If we put

$$
c=-\int_{0}^{\infty} e^{-\mu t} h(t) d t-\int_{0}^{\infty} e^{-\mu t} G(t) d t
$$

then

$$
H(x)=-\int_{x}^{\infty} e^{\mu(x-t)} h(t) d t-\int_{x}^{\infty} e^{\mu(x-t)} G(t) d t
$$

and therefore $H \in L^{2}[0, \infty)$.

Finally we will show that the triple $(a, b, c)$ given in (2) is unique. Let $\left(a_{1}, b_{1}, c_{1}\right) \neq$ $(a, b, c)$, and let $F_{1}, G_{1}, H_{1}$ be the functions corresponding to $\left(a_{1}, b_{1}, c_{1}\right)$. If $a_{1} \neq a$, then $F_{1}(x)=F(x)+\left(a_{1}-a\right) e^{\mu x}$. Thus $F_{1} \notin L^{2}[0, \infty)$, since $F \in L^{2}[0, \infty)$ and $e^{\mu x} \notin$ $L^{2}[0, \infty)$. If $a_{1}=a$ and $b_{1} \neq b$, then $G_{1}(x)=G(x)+\left(b_{1}-b\right) e^{\mu x}$, so $G_{1} \notin L^{2}[0, \infty)$. Similarly, if $a_{1}=a, b_{1}=b$ and $c_{1} \neq c$, then $H_{1} \notin L^{2}[0, \infty)$.

Recall that the characteristic polynomial for the matrix $A$ (given in $(3 \cdot 2))$ is $p(\mu)=$ $p(\mu ; \lambda)=\operatorname{det}(\mu I-A)=\left(\mu^{2}-\alpha^{2}+\lambda\right)^{2}\left(\mu^{2}-\alpha^{2}\right)+4 \mu^{2}$. If, for a given $\lambda$, no eigenvalue of $A$ is purely imaginary, then $\lambda \notin \mathbb{S}\left(M_{0}, N\right)$. In this case, the eigenvalues can be ordered: $\mu_{1}, \mu_{2}, \mu_{3},-\mu_{1},-\mu_{2},-\mu_{3}$, where $\operatorname{Re}\left(\mu_{j}\right)<0$ for $1 \leqslant j \leqslant 3$. There is a nonsingular matrix $S=S(\lambda)$ such that $S A S^{-1}=D$ is in Jordan canonical form. It is not hard to check that if $\lambda \neq \alpha^{2}$, then the $A$-annihilator of the vector $e_{1}=(1,0,0,0,0,0)^{T}$ coincides with $p(A)$. Therefore $p(\mu)$ is the minimal polynomial of $A$. This means that if $p(\mu)$ has multiple roots, then the matrix $D$ is not diagonal. There are three possibilities for the matrix $D$ (apart from permutation of the roots):

$$
D_{1}=\operatorname{diag}\left(\mu_{1}, \mu_{2}, \mu_{3}, \mu_{4}, \mu_{5}, \mu_{6}\right),
$$

where $\operatorname{Re}\left(\mu_{j}\right)<0$ for $1 \leqslant j \leqslant 3$,

$$
\mu_{4}=-\mu_{1}, \mu_{5}=-\mu_{2}, \mu_{6}=-\mu_{3},
$$

and $\mu_{i} \neq \mu_{j}$ for $i \neq j$, 


$$
\begin{aligned}
D_{2} & =\left(\begin{array}{cccccc}
\mu & 1 & 0 & 0 & 0 & 0 \\
0 & \mu & 0 & 0 & 0 & 0 \\
0 & 0 & \nu & 0 & 0 & 0 \\
0 & 0 & 0 & -\mu & 1 & 0 \\
0 & 0 & 0 & 0 & -\mu & 0 \\
0 & 0 & 0 & 0 & 0 & -\nu
\end{array}\right), \quad \text { where } \operatorname{Re}(\mu), \operatorname{Re}(\nu)<0, \text { and } \mu \neq \nu, \\
D_{3} & =\left(\begin{array}{cccccc}
\mu & 1 & 0 & 0 & 0 & 0 \\
0 & \mu & 1 & 0 & 0 & 0 \\
0 & 0 & \mu & 0 & 0 & 0 \\
0 & 0 & 0 & -\mu & 1 & 0 \\
0 & 0 & 0 & 0 & -\mu & 1 \\
0 & 0 & 0 & 0 & 0 & -\mu
\end{array}\right), \quad \text { where } \operatorname{Re}(\mu)<0
\end{aligned}
$$

Lemma $3 \cdot 2$. Let $D$ be one of the matrices (3·7)-(3·9). (We have assumed that no eigenvalue of $D$ is purely imaginary.) Consider the initial value problem:

$$
w^{\prime}-D w=f, \quad w(0)=w^{0}
$$

where

$$
\begin{gathered}
w(x)=\left(w_{1}(x), w_{2}(x), w_{3}(x), w_{4}(x), w_{5}(x), w_{6}(x)\right)^{T}, w^{0}=\left(w_{1}^{0}, w_{2}^{0}, w_{3}^{0}, w_{4}^{0}, w_{5}^{0}, w_{6}^{0}\right)^{T}, \\
f(x)=\left(f_{1}(x), f_{2}(x), f_{3}(x), f_{4}(x), f_{5}(x), f_{6}(x)\right)^{T} \text { and } f_{k} \in L^{2}[0, \infty) \text { for } 1 \leqslant k \leqslant 6 .
\end{gathered}
$$

The solution $w(x)$ has the following properties:

(1) for $1 \leqslant j \leqslant 3, w_{j} \in L^{2}[0, \infty)$ for all initial values $w_{1}^{0}, w_{2}^{0}, w_{3}^{0} \in \mathbb{C}$;

(2) for $4 \leqslant k \leqslant 6$, there are unique initial values $w_{4}^{0}, w_{5}^{0}, w_{6}^{0} \in \mathbb{C}$ so that $w_{k} \in L^{2}[0, \infty)$ for $4 \leqslant k \leqslant 6$. These values are the following:

(i) if $D=D_{1}$, then $w_{k}^{0}=-\int_{0}^{\infty} e^{-\mu_{k} t} f_{k}(t) d t$;

(ii) if $D=D_{2}$ then $w_{6}^{0}=-\int_{0}^{\infty} e^{\nu t} f_{6}(t) d t, w_{5}^{0}=-\int_{0}^{\infty} e^{\mu t} f_{5}(t) d t$ and $w_{4}^{0}=-\int_{0}^{\infty} e^{\mu t} f_{4}(t) d t+\int_{0}^{\infty} e^{\mu t}\left(\int_{t}^{\infty} e^{\mu(s-t)} f_{5}(s) d s\right) d t$

(iii) if $D=D_{3}$ then $w_{6}^{0}=-\int_{0}^{\infty} e^{\mu t} f_{6}(t) d t$,

$$
\begin{aligned}
w_{5}^{0}= & -\int_{0}^{\infty} e^{\mu t} f_{5}(t) d t+\int_{0}^{\infty} e^{\mu t}\left(\int_{t}^{\infty} e^{\mu(s-t)} f_{6}(s) d s\right) d t, \text { and } \\
w_{4}^{0}= & -\int_{0}^{\infty} e^{\mu t} f_{4}(t) d t+\int_{0}^{\infty} e^{\mu t}\left(\int_{t}^{\infty} e^{\mu(s-t)} f_{5}(s) d s\right) d t \\
& +\int_{0}^{\infty} e^{\mu t}\left[\int_{t}^{\infty} e^{\mu(s-t)}\left(\int_{s}^{\infty} e^{\mu(r-s)} f_{6}(r) d r\right) d s\right] d t
\end{aligned}
$$

Proof.

(i) If $D=D_{1}$, then the system of ODEs has the following form:

$$
w_{j}^{\prime}-\mu_{j} w_{j}=f_{j}(\text { for } 1 \leqslant j \leqslant 3), \quad w_{k}^{\prime}+\mu_{k} w_{k}=f_{k}(\text { for } 4 \leqslant k \leqslant 6) .
$$

The solution has the form:

$$
\begin{array}{ll}
w_{j}(x)=\int_{0}^{x} e^{\mu_{j}(x-t)} f_{j}(t) d t+w_{j}^{0} e^{\mu_{j} x} & (\text { for } 1 \leqslant j \leqslant 3), \\
w_{k}(x)=\int_{0}^{x} e^{-\mu_{k}(x-t)} f_{k}(t) d t+w_{k}^{0} e^{-\mu_{k} x} & (\text { for } 4 \leqslant k \leqslant 6) .
\end{array}
$$


(ii) If $D=D_{2}$, then the system of ODEs has the following form:

$$
\begin{array}{lll}
w_{1}^{\prime}-\mu w_{1}-w_{2}=f_{1}, & w_{2}^{\prime}-\mu w_{2}=f_{2}, & w_{3}^{\prime}-\nu w_{3}=f_{3}, \\
w_{4}^{\prime}+\mu w_{4}-w_{5}=f_{4}, & w_{5}^{\prime}+\mu w_{5}=f_{5}, & w_{6}^{\prime}+\nu w_{6}=f_{6} .
\end{array}
$$

The solution has the form:

$$
\begin{aligned}
& w_{3}(x)=\int_{0}^{x} e^{\nu(x-t)} f_{3}(t) d t+w_{3}^{0} e^{\nu x}, \quad w_{2}(x)=\int_{0}^{x} e^{\mu(x-t)} f_{2}(t) d t+w_{2}^{0} e^{\mu x}, \\
& w_{1}(x)=\int_{0}^{x} e^{\mu(x-t)} f_{1}(t) d t+\int_{0}^{x} e^{\mu(x-t)}\left(\int_{0}^{t} e^{\mu(t-s)} f_{2}(s) d s\right) d t+w_{2}^{0} x e^{\mu x}+w_{1}^{0} e^{\mu x}, \\
& w_{6}(x)=\int_{0}^{x} e^{-\nu(x-t)} f_{6}(t) d t+w_{6}^{0} e^{-\nu x}, \quad w_{5}(x)=\int_{0}^{x} e^{-\mu(x-t)} f_{5}(t) d t+w_{5}^{0} e^{-\mu x}, \\
& w_{4}(x)=\int_{0}^{x} e^{-\mu(x-t)} f_{4}(t) d t+\int_{0}^{x} e^{-\mu(x-t)}\left(\int_{0}^{t} e^{-\mu(t-s)} f_{5}(s) d s\right) d t+w_{5}^{0} x e^{-\mu x}+w_{4}^{0} e^{-\mu x} .
\end{aligned}
$$

(iii) If $D=D_{3}$, then the system of ODEs has the following form:

$$
\begin{array}{lll}
w_{1}^{\prime}-\mu w_{1}-w_{2}=f_{1}, & w_{2}^{\prime}-\mu w_{2}-w_{3}=f_{2}, & w_{3}^{\prime}-\mu w_{3}=f_{3}, \\
w_{4}^{\prime}+\mu w_{4}-w_{5}=f_{4}, & w_{5}^{\prime}+\mu w_{5}-w_{6}=f_{5}, & w_{6}^{\prime}+\mu w_{6}=f_{6} .
\end{array}
$$

The solution has the form

$$
\begin{aligned}
w_{3}(x)= & \int_{0}^{x} e^{\nu(x-t)} f_{3}(t) d t+w_{3}^{0} e^{\mu x}, \\
w_{2}(x)= & \int_{0}^{x} e^{\mu(x-t)} f_{2}(t) d t+\int_{0}^{x} e^{\mu(x-t)}\left(\int_{0}^{t} e^{\mu(t-s)} f_{3}(s) d s\right) d t+w_{3}^{0} x e^{\mu x}+w_{2}^{0} e^{\mu x}, \\
w_{1}(x)= & \int_{0}^{x} e^{\mu(x-t)} f_{1}(t) d t+\int_{0}^{x} e^{\mu(x-t)}\left(\int_{0}^{t} e^{\mu(t-s)} f_{2}(s) d s\right) d t \\
& +\int_{0}^{x} e^{\mu(x-t)}\left[\int_{0}^{t} e^{\mu(t-s)}\left(\int_{0}^{s} e^{\mu(s-r)} f_{3}(r) d r\right) d s\right] d t+\frac{w_{3}^{0}}{2} x^{2} e^{\mu x}+w_{2}^{0} x e^{\mu x}+w_{1}^{0} e^{\mu x}, \\
w_{6}(x)= & \int_{0}^{x} e^{-\nu(x-t)} f_{6}(t) d t+w_{6}^{0} e^{-\nu x}, \\
w_{5}(x)= & \int_{0}^{x} e^{-\mu(x-t)} f_{5}(t) d t+\int_{0}^{x} e^{-\mu(x-t)}\left(\int_{0}^{t} e^{-\mu(t-s)} f_{6}(s) d s\right) d t+w_{6}^{0} x e^{-\mu x}+w_{5}^{0} e^{-\mu x}, \\
w_{4}(x)= & \int_{0}^{x} e^{-\mu(x-t)} f_{4}(t) d t+\int_{0}^{x} e^{-\mu(x-t)}\left(\int_{0}^{t} e^{-\mu(t-s)} f_{5}(s) d s\right) d t \\
& +\int_{0}^{x} e^{-\mu(x-t)}\left[\int_{0}^{t} e^{-\mu(t-s)}\left(\int_{0}^{s} e^{-\mu(s-r)} f_{6}(r) d r\right) d s\right] d t \\
& +\frac{w_{6}^{0}}{2} x^{2} e^{-\mu x}+w_{5}^{0} x e^{-\mu x}+w_{4}^{0} e^{-\mu x} .
\end{aligned}
$$

The statements (1) and (2) follow from Lemma $3 \cdot 1$.

Recall that the equation $L_{0}(y, z)^{T}=(f, g)^{T}$ is equivalent to the system of ODEs $(3 \cdot 1)$ with boundary conditions $(3 \cdot 3)$. Let $S=S(\lambda)=\left(s_{i j}\right) \quad(1 \leqslant i, j \leqslant 6)$ be a matrix such that $S A S^{-1}=D$, where $D$ is one of the matrices $D_{1}, D_{2}, D_{3}$ displayed in 
$(3 \cdot 7)-(3 \cdot 9)$. If we put

$$
w=S\left(\begin{array}{l}
u \\
v
\end{array}\right)
$$

then $(3 \cdot 1)$ is equivalent to the system:

$$
w^{\prime}-D w=\left(\begin{array}{l}
s_{14} f-s_{16} g \\
s_{24} f-s_{26} g \\
s_{34} f-s_{36} g \\
s_{44} f-s_{46} g \\
s_{54} f-s_{56} g \\
s_{64} f-s_{66} g
\end{array}\right) .
$$

The following notation will be needed below. Let

$$
\begin{array}{cc}
T=S^{-1}=\left(t_{i j}\right) & (1 \leqslant i, j \leqslant 6), \\
T_{0}=\left(\begin{array}{ccc}
t_{11} & t_{12} & t_{13} \\
t_{21} & t_{22} & t_{23} \\
t_{51} & t_{52} & t_{53}
\end{array}\right), \quad T_{1}=\left(\begin{array}{lll}
t_{14} & t_{15} & t_{16} \\
t_{24} & t_{25} & t_{26} \\
t_{54} & t_{55} & t_{56}
\end{array}\right) .
\end{array}
$$

Corresponding to Lemma $3 \cdot 2(2)$, we define three continuous linear maps $\phi_{k}$ : $L^{2}[0, \infty) \rightarrow \mathbb{C}(4 \leqslant k \leqslant 6)$. If we set $f_{k}=f(4 \leqslant k \leqslant 6)$, then $\phi_{k}(f)=w_{k}^{0}$ in Lemma $3 \cdot 2(2)$. Thus:

(i) if $D=D_{1}$, then $\phi_{k}(f)=-\int_{0}^{\infty} e^{\mu_{k} t} f(t) d t$;

(ii) if $D=D_{2}$ then $\phi_{6}(f)=-\int_{0}^{\infty} e^{\nu t} f(t) d t, \phi_{5}(f)=-\int_{0}^{\infty} e^{\mu t} f(t) d t$, and

$$
\phi_{4}(f)=-\int_{0}^{\infty} e^{\mu t} f(t) d t+\int_{0}^{\infty} e^{\mu t}\left(\int_{t}^{\infty} e^{\mu(s-t} f(s) d s\right) d t ;
$$

(iii) if $D=D_{3}$ then $\phi_{6}(f)=-\int_{0}^{\infty} e^{\mu t} f(t) d t$,

$$
\begin{aligned}
\phi_{5}(f)= & -\int_{0}^{\infty} e^{\mu t} f(t) d t+\int_{0}^{\infty} e^{\mu t}\left(\int_{t}^{\infty} e^{\mu(s-t} f(s) d s\right) d t \text { and } \\
\phi_{4}(f)= & -\int_{0}^{\infty} e^{\mu t} f(t) d t+\int_{0}^{\infty} e^{\mu t}\left(\int_{t}^{\infty} e^{\mu(s-t} f(s) d s\right) d t \\
& \left.\left.+\int_{0}^{\infty} e^{\mu t}\left[\int_{t}^{\infty} e^{\mu(s-t}\right) \int_{s}^{\infty} e^{\mu(r-s)} f(r) d r\right) d s\right] d t .
\end{aligned}
$$

We define a continuous linear map $\psi: Y \rightarrow \mathbb{C}^{3}$ as follows:

$$
\psi(f, g)=T_{1}\left(\begin{array}{l}
s_{44} \phi_{4}(f)-s_{46} \phi_{4}(g) \\
s_{54} \phi_{5}(f)-s_{56} \phi_{5}(g) \\
s_{64} \phi_{6}(f)-s_{66} \phi_{6}(g)
\end{array}\right) .
$$

Observe that the sets $\mathcal{U}$ and $\mathcal{V}$ defined by

$$
\mathcal{U}=\psi(Y) \text { and } \quad \mathcal{V}=\text { Range }\left(T_{0}\right)
$$

are subspaces of $\mathbb{C}^{3}$. Recall that $\lambda$ is an eigenvalue for $\left(M_{0}, N\right)$ if the operator $L_{0}=$ $M_{0}-\lambda N$ is not injective.

Theorem 3·5. Suppose that $\lambda \notin \mathbb{S}\left(M_{0}, N\right)$. Then:

(1) $(f, g) \in$ Range $\left(L_{0}\right)$ if and only if $\psi(f, g) \in U \cap \mathcal{V}$;

(2) $\operatorname{def}\left(L_{0}\right)=\operatorname{dim}(\mathcal{U} /(\mathcal{U} \cap \mathcal{V}))$;

(3) $\lambda$ is an eigenvalue for $\left(M_{0}, N\right)$ if and only if $\operatorname{det} T_{0}(\lambda)=0$;

(Here $T_{0}$ is the $3 \times 3$ matrix defined in $(3 \cdot 12)$.)

(4) $\operatorname{null}\left(L_{0}\right)=\operatorname{null}\left(T_{0}\right)$;

(5) $L_{0}$ is Fredholm. 
Proof. Since $w=S\left(\begin{array}{l}u \\ v\end{array}\right),\left(\begin{array}{l}u \\ v\end{array}\right)=T(w)$, and so

$$
\left(\begin{array}{l}
u_{1}(0) \\
u_{2}(0) \\
v_{1}(0)
\end{array}\right)=T_{0}\left(\begin{array}{l}
w_{1}(0) \\
w_{2}(0) \\
w_{3}(0)
\end{array}\right)+T_{1}\left(\begin{array}{l}
w_{4}(0) \\
w_{5}(0) \\
w_{6}(0)
\end{array}\right) .
$$

Thus the boundary conditions for $(3 \cdot 10)$ are:

$$
T_{0}\left(\begin{array}{l}
w_{1}(0) \\
w_{2}(0) \\
w_{3}(0)
\end{array}\right)+T_{1}\left(\begin{array}{l}
w_{4}(0) \\
w_{5}(0) \\
w_{6}(0)
\end{array}\right)=0
$$

and $w_{k} \in L^{2}[0, \infty)$ for $1 \leqslant k \leqslant 6$.

For $1 \leqslant j \leqslant 3$, Lemma $3 \cdot 2(1)$ says that $w_{j} \in L^{2}[0, \infty)$ for any choice of $w_{j}(0)$. By $(3 \cdot 10)$ and Lemma $3 \cdot 2(2), w_{k} \in L^{2}[0, \infty)$ for $4 \leqslant k \leqslant 6$ if and only if

$$
w_{k}(0)=s_{k 4} \phi_{k}(f)-s_{k 6} \phi_{k}(g), \text { for } 4 \leqslant k \leqslant 6 .
$$

Therefore, by $(3 \cdot 13)-(3 \cdot 15),(f, g) \in$ Range $\left(L_{0}\right)$ if and only if $\psi(f, g) \in \mathcal{U} \cap \mathcal{V}$. This proves (1). Since $\psi: Y \rightarrow \mathcal{U}$ is a continuous linear map, and $\operatorname{dim}(\mathcal{U} \cap \mathcal{V})<\infty$, it also follows that Range $\left(L_{0}\right)$ is closed.

Since $\psi(Y)=\mathcal{U}$, and $\psi\left(\operatorname{Range}\left(L_{0}\right)\right)=\mathcal{U} \cap \mathcal{V}$, we see that

$$
\operatorname{def}\left(L_{0}\right)=\operatorname{dim}(\mathcal{U} /(\mathcal{U} \cap \mathcal{V}))
$$

This proves (2).

$\lambda$ is an eigenvalue for $\left(M_{0}, N\right)$ if and only if the system $w^{\prime}-D w=0$ has a nontrivial solution $w \in\left(L^{2}[0, \infty)\right)^{6}$ satisfying the boundary conditions $(3 \cdot 15)$. By Lemma $3 \cdot 2(2)$ $w_{k}(0)=0$ for $4 \leqslant k \leqslant 6$. Thus the boundary conditions in this case are:

$$
T_{0}\left(\begin{array}{l}
w_{1}(0) \\
w_{2}(0) \\
w_{3}(0)
\end{array}\right)=0
$$

This proves (3) and (4). We have shown that Range $\left(L_{0}\right)$ is closed, null $\left(L_{0}\right)<\infty$ and $\operatorname{def}\left(L_{0}\right)<\infty$. Therefore $L_{0}$ is Fredholm.

The following theorem follows from Corollary $3 \cdot 1$ and Theorems $3 \cdot 3-3 \cdot 5$. This is the first main result in this paper.

Theorem 3.6. $\sigma_{\text {ess }}(M, N)=\mathbb{S}\left(M_{0}, N\right)=\left\{\nu+i \eta: \eta^{2}=4\left(1-\alpha^{2} / \nu\right), \quad \nu \geqslant \alpha^{2}\right\}($ see equation $(3 \cdot 4))$.

The following important sets will often occur in the ensuing sections.

Definition $3 \cdot 1$.

$$
\Omega(M, N)=\mathbb{C} \backslash\left(B \cup \sigma_{e s s}(M, N)\right),
$$

where $B$ is the set of branch points of the characteristic polynomial $p(\mu)$. (i.e. This is the set where $p(\mu)$ has multiple roots.) $\Omega(M, N)$ has two connected components:

$$
\Omega(M, N)=\Omega_{+}(M, N) \cup \Omega_{-}(M, N),
$$


where $\Omega_{+}(M, N)$ is the component containing the interval $\left(\alpha^{2}, \infty\right) \subset \mathbb{R}$, and $\Omega_{-}(M, N)$ contains the interval $\left(-\infty, \alpha^{2}\right)$.

\section{4. $L^{2}$ and non- $L^{2}$ solutions as analytic functions}

In Section 7 below, we develop the Titchmarsh-Weyl coefficients. These provide a further characterization of isolated eigenvalues, and allow us to examine the effects of regularizing the Ekman problem by replacing it by a problem on a finite interval. In order to develop the Titchmarsh-Weyl coefficients we examine here the $L^{2}$ solutions of the system of differential equations $(2 \cdot 14)$, which we now write as a perturbation of the constant coefficient system of Section 3 :

$$
Y^{\prime}=(A(\lambda)+Q(x)) Y, \quad x \in[0, \infty),
$$

where $A(\lambda)$ is given in $(3 \cdot 2)$. We shall assume that $\lambda \in \Omega(M, N)$ (see Definition $3 \cdot 1$ ). Then the matrix $A(\lambda)$ possesses 6 distinct eigenvalues $\mu_{1}(\lambda), \ldots, \mu_{6}(\lambda)$, with associated eigenvectors $v_{1}(\lambda), \ldots, v_{6}(\lambda)$. The $\mu_{j}$ are ordered so that

$$
\operatorname{Re}\left(\mu_{1}\right), \operatorname{Re}\left(\mu_{2}\right), \operatorname{Re}\left(\mu_{3}\right)<0, \quad \operatorname{Re}\left(\mu_{4}\right), \operatorname{Re}\left(\mu_{5}\right), \operatorname{Re}\left(\mu_{6}\right)>0 .
$$

The $\mu_{j}(\lambda)$ are values of a multivalued algebraic function. However, they are singlevalued analytic functions in any disc $D \subset \Omega(M, N)$. We shall sometimes express this by saying that the $\mu_{j}(\lambda)$ are "locally analytic" in $\Omega(M, N)$. By assumption (A1·3) the matrix $Q$ lies in $L^{1}[0, \infty)$.

We denote by $T(\lambda)$ the invertible (locally) analytic $6 \times 6$ matrix with columns $v_{1}, \ldots, v_{6}$. This is the same as the matrix given in $(3 \cdot 11)$. Making the transformation

$$
Y=T(\lambda) Z
$$

reduces $(4 \cdot 1)$ to the form

$$
Z^{\prime}=(D(\lambda)+\hat{Q}(x, \lambda)) Z
$$

in which $D(\lambda)=\operatorname{diag}\left(\mu_{1}(\lambda), \ldots, \mu_{6}(\lambda)\right)$ and $\hat{Q}(x, \lambda)=T(\lambda)^{-1} Q(x) T(\lambda)$. We define

$$
\begin{aligned}
& \Phi_{1}(x, \lambda)=\operatorname{diag}\left(\mathrm{e}^{\mu_{1}(\lambda) x}, \mathrm{e}^{\mu_{2}(\lambda) x}, \mathrm{e}^{\mu_{3}(\lambda) x}, 0,0,0\right), \\
& \Phi_{2}(x, \lambda)=\operatorname{diag}\left(0,0,0, \mathrm{e}^{\mu_{4}(\lambda) x}, \mathrm{e}^{\mu_{5}(\lambda) x}, \mathrm{e}^{\mu_{6}(\lambda) x}\right)
\end{aligned}
$$

and

$$
\Phi(x, \lambda)=\Phi_{1}(x)+\Phi_{2}(x) .
$$

It is easy to check that $\Phi$ is a fundamental matrix for the differential system

$$
Z^{\prime}=D(\lambda) Z
$$

Define

$$
\Phi_{6 \times 3}(x, \lambda)=\left(\begin{array}{ccc}
\mathrm{e}^{\mu_{1}(\lambda) x} & 0 & 0 \\
0 & \mathrm{e}^{\mu_{2}(\lambda) x} & 0 \\
0 & 0 & \mathrm{e}^{\mu_{3}(\lambda) x} \\
0 & 0 & 0 \\
0 & 0 & 0 \\
0 & 0 & 0
\end{array}\right) .
$$


Using the variation of parameters formula, it may be verified that for any $a \geqslant 0$, any matrix function $Z$ which satisfies an equation of the form

$$
\begin{aligned}
Z(x, \lambda)= & \Phi_{1}(x, \lambda) \int_{a}^{x} \Phi(t, \lambda)^{-1} \hat{Q}(t, \lambda) Z(t, \lambda) d t \\
& -\Phi_{2}(x, \lambda) \int_{x}^{\infty} \Phi(t, \lambda)^{-1} \hat{Q}(t, \lambda) Z(t, \lambda) d t+\Phi_{6 \times 3}(x, \lambda),
\end{aligned}
$$

will be a solution of $(4 \cdot 4)$. We use this fact to construct a $6 \times 3$ solution matrix of $(4 \cdot 4)$ which is (locally) analytic in $\lambda$ and whose columns span the space of $L^{2}[0, \infty)$ solutions of $(4 \cdot 4)$. Note that from the Levinson Theorem [10, theorem 1.3] we already know that the space of $L^{2}[0, \infty)$ solutions of $(4 \cdot 4)$ is three-dimensional and contains solutions with the asymptotic behaviour

$$
Z_{k}(x)=\left(\mathrm{e}_{k}+o(1)\right) \exp \left(\mu_{k} x\right), x \longrightarrow \infty, \quad k=1,2,3,
$$

where the $\mathrm{e}_{k}$ are the standard basis vectors of $\mathbb{C}^{6}$.

Let $a \geqslant 0$ be fixed, and let $B C_{6 \times 3}[a, \infty)$ denote the space of all continuous $6 \times 3$ matrix functions $Z$ on $[a, \infty)$ with

$$
\sup _{x \in[a, \infty)}\|Z(x)\|<+\infty .
$$

We define a map $\Sigma_{\lambda}: B C_{6 \times 3}[a, \infty) \rightarrow B C_{6 \times 3}[a, \infty)$ by

$$
\begin{aligned}
\Sigma_{\lambda}(Z)(x)= & \Phi_{1}(x, \lambda) \int_{a}^{x} \Phi(t, \lambda)^{-1} \hat{Q}(t, \lambda) Z(t) d t \\
& -\Phi_{2}(x, \lambda) \int_{x}^{\infty} \Phi(t, \lambda)^{-1} \hat{Q}(t, \lambda) Z(t) d t+\Phi_{6 \times 3}(x, \lambda) .
\end{aligned}
$$

It is not immediately obvious that $\Sigma_{\lambda}$ maps into $B C_{6 \times 3}[a, \infty)$. To see this we observe that

$$
\begin{aligned}
& \Phi_{1}(x) \Phi(t)^{-1}=\operatorname{diag}\left(\mathrm{e}^{\mu_{1}(\lambda)(x-t)}, \mathrm{e}^{\mu_{2}(\lambda)(x-t)}, \mathrm{e}^{\mu_{3}(\lambda)(x-t)}, 0,0,0\right), \\
& \Phi_{2}(x) \Phi(t)^{-1}=\operatorname{diag}\left(0,0,0, \mathrm{e}^{\mu_{4}(\lambda)(x-t)}, \mathrm{e}^{\mu_{5}(\lambda)(x-t)}, \mathrm{e}^{\mu_{6}(\lambda)(x-t)}\right) .
\end{aligned}
$$

These expressions, together with the assumptions $\operatorname{Re}\left(\mu_{1}, \mu_{2}, \mu_{3}\right)<0, \operatorname{Re}\left(\mu_{4}, \mu_{5}, \mu_{6}\right)>$ 0 , give

$$
\left.\begin{array}{l}
\left\|\Phi_{1}(x) \Phi(t)^{-1}\right\| \leqslant 1, \quad x \geqslant t \\
\left\|\Phi_{2}(x) \Phi(t)^{-1}\right\| \leqslant 1, \quad t \geqslant x .
\end{array}\right\}
$$

Furthermore, since $Q \in L^{1}[0, \infty)$ it follows that

$$
\int_{0}^{\infty}\|\hat{Q}(t, \lambda)\| d t<+\infty
$$

Equations (4.13) and (4.14) together with the fact that

$$
\lim _{x \rightarrow \infty}\left\|\Phi_{6 \times 3}(x, \lambda)\right\|=0
$$

ensure that $\Sigma_{\lambda}$ does indeed map into $B C_{6 \times 3}[a, \infty)$.

Now suppose that $\lambda$ lies in some compact set $\mathcal{K}$ (which we assume is a closed disc $\subset$ $\Omega(M, N))$. Exploiting $(4 \cdot 14)$, choose $a$ such that

$$
\int_{a}^{\infty}\|\hat{Q}(t, \lambda)\| d t<1 \quad \forall \lambda \in \mathcal{K} .
$$


Given any two maps $Z_{1}$ and $Z_{2}$ in $B C_{6 \times 3}[a, \infty)$, the triangle inequality applied to $(4 \cdot 11)$, together with $(4 \cdot 13)$, yields

$$
\begin{aligned}
\left\|\Sigma_{\lambda}\left(Z_{1}\right)(x)-\Sigma_{\lambda}\left(Z_{2}\right)(x)\right\| \leqslant & \left(\int_{a}^{x}\|\hat{Q}(t, \lambda)\| d t\right) \sup _{t \in[a, x]}\left\|Z_{1}(t)-Z_{2}(t)\right\| \\
& +\left(\int_{x}^{\infty}\|\hat{Q}(t, \lambda)\| d t\right) \sup _{t \in[x, \infty]}\left\|Z_{1}(t)-Z_{2}(t)\right\|,
\end{aligned}
$$

which simplifies to

$$
\left\|\Sigma_{\lambda}\left(Z_{1}\right)(x)-\Sigma_{\lambda}\left(Z_{2}\right)(x)\right\| \leqslant\left(\int_{a}^{\infty}\|\hat{Q}(t, \lambda)\| d t\right) \sup _{t \in[a, \infty]}\left\|Z_{1}(t)-Z_{2}(t)\right\| .
$$

As the right-hand side in $(4 \cdot 16)$ does not depend on $x$, this inequality, together with $(4 \cdot 15)$, shows that for $\lambda \in \mathcal{K}$ the map $\Sigma_{\lambda}$ is a contraction on $B C_{6 \times 3}[a, \infty)$. Thus $\Sigma_{\lambda}$ possesses a unique fixed point in $B C_{6 \times 3}[a, \infty)$, which is the bounded $6 \times 3$ matrix solution of (4.9). We denote this solution by $Z_{\text {bound }}$.

The columns of $Z_{\text {bound }}$ are $L^{2}$ solutions of the differential equation (4.4). This is because non- $L^{2}$ solutions of $(4 \cdot 4)$ blow up at infinity. In fact, by the Levinson Theorem [10, theorem 1·3] three linearly independent non- $L^{2}$ solutions of $(4 \cdot 1)$ can be constructed containing elements with the asymptotic behaviour

$$
Z_{k}(x)=\left(\mathrm{e}_{k}+o(1)\right) \exp \left(\mu_{k} x\right), \quad x \longrightarrow \infty, \quad k=4,5,6 .
$$

Moreover, the columns of $Z_{\text {bound }}$ form a basis for the space of $L^{2}$ solutions. To see this it is sufficient to show that $Z_{\text {bound }}$ is of full rank. If there were some 3 -vector c such that $Z_{\text {bound }} \mathbf{c}=\mathbf{0}$ then multiplying both sides of $(4 \cdot 9)$ on the right by $\mathbf{c}$ would give

$$
\Phi_{6 \times 3}(x, \lambda) \mathbf{c}=\mathbf{0} .
$$

However since $\Phi_{6 \times 3}$ is of full rank, this implies $\mathbf{c}=\mathbf{0}$. Thus $Z_{\text {bound }}$ is also of full rank. Finally, since $\Sigma_{\lambda}$ is an analytic contraction for $\lambda \in \mathcal{K}$, it follows that $Z_{\text {bound }}$ is also analytic for $\lambda \in \mathcal{K}$. (The fixed point is the limit of a $\Sigma_{\lambda}$-orbit: $Z_{n+1}=\Sigma_{\lambda}\left(Z_{n}\right)$. This is a uniformly convergent sequence of analytic functions.) Transforming back to the original variables via $(4 \cdot 3)$, we have proved the following result.

Theorem $4 \cdot 1$. Let $\mathcal{K} \subset \Omega(M, N)$ be a closed disc. Then the differential equation $(4 \cdot 1)$ possesses a $6 \times 3$ solution matrix $Y_{\text {bound }}(x, \lambda)$ which is analytic in $\lambda \in \mathcal{K}$ and whose columns span the space of $L^{2}$ solutions of $(4 \cdot 1)$.

Remark 2. While we require $x \in[a, \infty)$ for the contraction argument, the solution for $x \in[0, \infty)$ is given by the same formula $(4 \cdot 9)$.

In addition to the analytic matrix $Y_{\text {bound }}$ it will be necessary in our development of the Titchmarsh-Weyl coefficients in Section 7 to have an analytic basis for the remainder of the solution space of $(4 \cdot 1)$. This part of the solution space contains functions which are not bounded, so the argument used above fails. Nevertheless one can still use the method of Eastham [10] to obtain a result similar to Theorem $4 \cdot 1$.

Theorem 4.2. Let $\mathcal{K}$ be a compact subset of a disc $D \subset \Omega(M, N)$, such that $\operatorname{Re} \mu_{4}(\lambda) \leqslant \operatorname{Re} \mu_{5}(\lambda) \leqslant \operatorname{Re} \mu_{6}(\lambda)$ for $\lambda \in \mathcal{K}$. Then the equation (4.1) possesses solutions 
$Y_{4}, Y_{5}$ and $Y_{6}$ which are continuous on $\mathcal{K}$, analytic in the interior of $\mathcal{K}$, and which have the asymptotic expansions

$$
Y_{j}(x, \lambda)=\exp \left(\mu_{j}(\lambda) x\right)\left\{v_{j}+o(1)\right\}, \quad j=4,5,6,
$$

uniformly for $\lambda \in \mathcal{K}$ as $x \rightarrow \infty$.

Proof. It is sufficient to show that (4.4) has solutions $Z_{4}, Z_{5}$ and $Z_{6}$ which have the asymptotic behaviour

$$
Z_{j}(x, \lambda)=\exp \left(\mu_{j}(\lambda) x\right)\left\{\mathbf{e}_{j}+o(1)\right\}, \quad j=4,5,6,
$$

for large positive $x$. To do this, fix $j(4 \leqslant j \leqslant 6)$, and for a solution $Z$ of $(4 \cdot 4)$ define a new variable $\tilde{Z}$ by

$$
\tilde{Z}(x, \lambda)=Z(x, \lambda) \exp \left(-\mu_{j}(\lambda) x\right) .
$$

Then $\tilde{Z}$ satisfies the differential equation

$$
\tilde{Z}^{\prime}=(\tilde{D}(\lambda)+\hat{Q}(x, \lambda)) \tilde{Z},
$$

in which

$$
\tilde{D}=\operatorname{diag}\left(\mu_{1}-\mu_{j}, \ldots, \mu_{6}-\mu_{j}\right) .
$$

Following Eastham [10, p. 11], define

$$
\begin{gathered}
\Phi_{1}(x, \lambda)=\operatorname{diag}\left(\exp \left(\left(\mu_{1}-\mu_{j}\right) x\right), \ldots, \exp \left(\left(\mu_{j-1}-\mu_{j}\right) x\right), 0, \cdots, 0\right), \\
\Phi_{2}(x, \lambda)=\operatorname{diag}\left(0, \ldots, 0,1, \exp \left(\left(\mu_{j+1}-\mu_{j}\right) x\right), \ldots, \exp \left(\left(\mu_{6}-\mu_{j}\right) x\right)\right), \\
\Phi=\Phi_{1}+\Phi_{2} .
\end{gathered}
$$

Following the approach which we used for Theorem $4 \cdot 1$ it can be shown that $(4 \cdot 19)$ possesses a solution $\tilde{Z}_{j}$ which satisfies

$$
\tilde{Z}_{j}(x, \lambda) \longrightarrow \mathbf{e}_{j} \text { uniformly for } \lambda \in \mathcal{K} \text { as } x \longrightarrow \infty,
$$

and is a fixed point of the mapping

$$
Z \longmapsto \mathbf{e}_{j}+\Phi_{1}(x, \lambda) \int_{a}^{x} \Phi^{-1}(t) \hat{Q}(t, \lambda) Z(t) d t-\Phi_{2}(x, \lambda) \int_{x}^{\infty} \Phi^{-1}(t) \hat{Q}(t, \lambda) Z(t) d t .
$$

Provided $a$ is chosen to satisfy (4-15) for $\lambda \in \mathcal{K}$, this map is an analytic contraction, and so its fixed point, which we denote $\tilde{Z}_{j}(x, \lambda)$, is continuous on $\mathcal{K}$ and analytic in the interior of $\mathcal{K}$ (where $\operatorname{Re} \mu_{4}(\lambda)<\operatorname{Re} \mu_{5}(\lambda)<\operatorname{Re} \mu_{6}(\lambda)$ ). The corresponding solution of (4.4) we denote by $Z_{j}(x, \lambda)$ and is given, according to (4·18), by

$$
Z_{j}(x, \lambda)=\exp \left(\mu_{j}(\lambda) x\right) \tilde{Z}_{j}(x, \lambda),
$$

and, from $(4 \cdot 20)$, this solution $Z_{j}$ must have the property $(4 \cdot 17)$. This completes the proof.

Remark 3 . The vectors $v_{4}, v_{5}, v_{6}$ in Theorem 4.2 are the last three columns of the matrix $T(\lambda)$ in $(4 \cdot 3)$.

\section{A-priori information about the spectrum}

Both for theoretical and for computational purposes, it is very useful to have apriori information about the spectrum. In this section we show that all points of the 
spectrum of an Ekman problem lie in a right half-plane, whether the problem be posed on a finite or a semi-infinite interval. The methods used are essentially those used by Lahmann and Plum [17] for the Orr-Sommerfeld equation. Assumptions $(\mathrm{A} 1 \cdot 1-\mathrm{A} 1 \cdot 3)$ are needed to make these methods work.

We start as in Section 3 by writing the Ekman problem as

$$
(M \zeta)(x)=\lambda(N \zeta)(x), \quad x \in[0, \infty),
$$

with $\zeta=(y, z)^{T}$ and boundary conditions $y(0)=y^{\prime}(0)=z(0)=0$. We wish to compute the quadratric forms associated with $M$ and $N$. Integration by parts using the boundary condition $y(0)=0$ shows that the quadratic form associated with $N$ satisfies

$$
N(\zeta, \zeta)=\int_{0}^{\infty} \zeta^{*} N \zeta d x=\left\|y^{\prime}\right\|_{L^{2}}^{2}+\alpha^{2}\|y\|_{L^{2}}^{2}+\|z\|_{L^{2}}^{2}-\lim _{x \rightarrow \infty} \bar{y}(x) y^{\prime}(x) .
$$

We now establish that $\lim _{x \rightarrow \infty} \bar{y}(x) y^{\prime}(x)=0$. Because $y \in H^{2}[0, \infty)$, we know that both $y$ and $y^{\prime}$ lie in $L^{2}[0, \infty)$, and hence $\bar{y} y^{\prime} \in L^{1}[0, \infty)$. Moreover,

$$
\lim _{x \rightarrow \infty} \bar{y}(x) y^{\prime}(x)=\bar{y}(0) y^{\prime}(0)+\int_{0}^{\infty}\left(\bar{y} y^{\prime}\right)^{\prime}=\int_{0}^{\infty}\left\{\left|y^{\prime}\right|^{2}+\bar{y} y^{\prime \prime}\right\},
$$

and the integral converges because $y \in H^{2}[0, \infty)$. Thus $\bar{y} y^{\prime}$ has a finite limit at infinity and lies in $L^{1}[0, \infty)$. Necessarily, therefore,

$$
\lim _{x \rightarrow \infty} \bar{y}(x) y^{\prime}(x)=0,
$$

and we obtain

$$
N(\zeta, \zeta)=\int_{0}^{\infty} \zeta^{*} N \zeta d x=\left\|y^{\prime}\right\|_{L^{2}}^{2}+\alpha^{2}\|y\|_{L^{2}}^{2}+\|z\|_{L^{2}}^{2} .
$$

Integration by parts also allows us to show that the quadratic form associated with $M$ is

$$
\begin{aligned}
M(\zeta, \zeta)= & \int_{0}^{\infty} \zeta^{*} M \zeta d x=\left\|y^{\prime \prime}\right\|_{L^{2}}^{2}+2 \alpha^{2}\left\|y^{\prime}\right\|_{L^{2}}^{2}+\alpha^{4}\|y\|_{L^{2}}^{2}+\left\|z^{\prime}\right\|_{L^{2}}^{2}+\alpha^{2}\|z\|_{L^{2}}^{2} \\
& +\int_{0}^{\infty}\left\{2\left(y^{\prime} \bar{z}+z^{\prime} \bar{y}\right)+i \alpha R V\left(\alpha^{2}|y|^{2}+|z|^{2}\right)+i \alpha R V^{\prime \prime}|y|^{2}-i \alpha R V y^{\prime \prime} \bar{y}\right. \\
& \left.+i \alpha R U^{\prime} y \bar{z}\right\} d x .
\end{aligned}
$$

This time, the boundary terms involve the limits

$$
\lim _{x \rightarrow \infty} \overline{y^{\prime \prime \prime}}(x) y(x), \quad \lim _{x \rightarrow \infty} \overline{y^{\prime \prime}}(x) y^{\prime}(x) .
$$

Since $y \in H^{4}[0, \infty)$, we know that $y^{\prime \prime \prime} \in L^{2}[0, \infty)$ and $y \in L^{2}[0, \infty)$, and so $\overline{y^{\prime \prime \prime}} y \in$ $L^{1}[0, \infty)$. Moreover,

$$
\lim _{x \rightarrow \infty} \overline{y^{\prime \prime \prime}}(x) y(x)=\overline{y^{\prime \prime \prime}}(0) y(0)+\int_{0}^{\infty}\left(\overline{y^{\prime \prime \prime}} y\right)^{\prime}=\int_{0}^{\infty}\left\{\overline{y^{(i v)}} y+\overline{y^{\prime \prime \prime}} y^{\prime}\right\},
$$

and the integral converges because $y^{(i v)}, y^{\prime \prime \prime}, y^{\prime}$ and $y$ all lie in $L^{2}[0, \infty)$. Thus $\overline{y^{\prime \prime \prime}} y$ lies in $L^{1}[0, \infty)$ and has finite limit at infinity, so necessarily

$$
\lim _{x \rightarrow \infty} \overline{y^{\prime \prime \prime}}(x) y(x)=0 .
$$

Other boundary terms can be treated similarly. 
We now bound various terms on the right-hand side of $(5 \cdot 2)$ in terms of the quadratic form associated with $N$. The bounds which we require are as follows:

$$
\begin{gathered}
\left|\alpha R \int_{0}^{\infty} V\left(\alpha^{2}|y|^{2}+|z|^{2}\right) d x\right| \leqslant \alpha R\|V\|_{L^{\infty}} N(\zeta, \zeta) ; \\
\left.\left|\alpha R \int_{0}^{\infty} V^{\prime \prime}\right| y\right|^{2} d x \mid \leqslant \frac{R\left\|V^{\prime \prime}\right\|_{L^{\infty}}}{\alpha} N(\zeta, \zeta) ; \\
\alpha R\left|\int_{0}^{\infty} V y^{\prime \prime} \bar{y} d x\right| \\
=\alpha R\left|\int_{0}^{\infty} y^{\prime}(V \bar{y})^{\prime} d x\right| \\
\quad=\alpha R\left|\int_{0}^{\infty}\left(V^{\prime} y^{\prime} \bar{y}+V\left|y^{\prime}\right|^{2}\right) d x\right| \\
\leqslant \alpha R \frac{\left\|V^{\prime}\right\|_{L^{\infty}}}{2}\left(\left\|y^{\prime}\right\|_{L^{2}}^{2}+\|y\|_{L^{2}}^{2}\right)+\alpha R\|V\|_{L^{\infty}}\left\|y^{\prime}\right\|_{L^{2}}^{2} \\
\leqslant R\left\{\frac{\left\|V^{\prime}\right\|_{L^{\infty}}}{2}(\alpha+1 / \alpha)+\alpha\|V\|_{L^{\infty}}\right\} N(\zeta, \zeta) ; \\
\alpha R\left|\int_{0}^{\infty} U^{\prime} y \bar{z} d x\right| \leqslant \frac{R\left\|U^{\prime}\right\|_{L^{\infty}}}{2}(\alpha+1 / \alpha) N(\zeta, \zeta) ;
\end{gathered}
$$

and finally

$$
\left|\int_{0}^{\infty}\left(y^{\prime} \bar{z}+\overline{y^{\prime}} z\right) d x\right|=\left|\int_{0}^{\infty}\left(y^{\prime} \bar{z}-\bar{y} z^{\prime}\right) d x\right|=\left|2 i \operatorname{Im}\left(\int_{0}^{\infty} y^{\prime} \bar{z}\right)\right| \leqslant N(\zeta, \zeta) .
$$

In these inequalities, we use the shorthand $\|\cdot\|_{L^{\infty}}$ for $\|\cdot\|_{L^{\infty}[0, \infty)}$. Now if $\lambda$ is an eigenvalue then

$$
\lambda=M(\zeta, \zeta) / N(\zeta, \zeta)
$$

where $\zeta=(y, z)^{T}$ is the associated eigenfunction. Using the above inequalities and the expression for $M(\zeta, \zeta)$ in $(5 \cdot 2)$ we obtain the following result.

Theorem 5·1. Any eigenvalue $\lambda=\nu+i \mu$ satisfies

$$
\begin{gathered}
\nu \geqslant \alpha^{2}-\frac{R}{2}\left(\left\|U^{\prime}\right\|_{L^{\infty}}+\left\|V^{\prime}\right\|_{L^{\infty}}\right)(\alpha+1 / \alpha)-\alpha R\|V\|_{L^{\infty}}, \\
|\eta| \leqslant 2+2 \alpha R\|V\|_{L^{\infty}}+\frac{R}{2}\left(\left\|U^{\prime}\right\|_{L^{\infty}}+\left\|V^{\prime}\right\|_{L^{\infty}}\right)(\alpha+1 / \alpha)+\frac{R}{\alpha}\left\|V^{\prime \prime}\right\|_{L^{\infty}} .
\end{gathered}
$$

Theorem $5 \cdot 1$ locates the eigenvalues of the Ekman problem on $[0, \infty)$ within a semiinfinite strip. It is easy to check that the essential spectrum found in Section 3 also lies within this region, which therefore encloses the whole spectrum since the residual spectrum is empty.

If we consider the Ekman problem on any finite interval $[0, X]$ with boundary conditions

$$
y(0)=y^{\prime}(0)=z(0)=0, \quad y(X)=y^{\prime}(X)=z(X)=0,
$$

then similar bounds are obtained by precisely the same methods. 
Theorem 5.2. The eigenvalues of the Ekman problem on any finite interval $[0, X]$

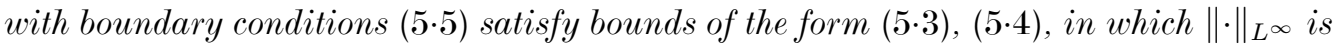
now to be interpreted as $\|\cdot\|_{L^{\infty}[0, X]}$.

What about the essential spectrum for a problem on $[0, X]$ ? Not surprisingly, the essential spectrum of such a problem is empty. In fact, we now show that the resolvent is the whole of $\mathbb{C}$ minus a set of isolated points.

Theorem 5.3. The spectrum of the Ekman problem on $[0, X]$ with the boundary conditions (5.5) consists of a discrete set of isolated eigenvalues having no finite limit points. In particular, there are no open sets of eigenvalues and there is no essential spectrum.

Proof. For a differential equation problem on a finite interval the points of the resolvent are those values of $\lambda$ for which the characteristic determinant is nonzero. The characteristic determinant is an entire function of $\lambda$; if its zeros had a finite limit point then the characteristic determinant would be identically zero throughout $\mathbb{C}$. Every $\lambda \in \mathbb{C}$ would then be an eigenvalue of the problem, contradicting the bounds in Theorem $5 \cdot 2$.

We remark that the positivity of the quadratic form associated with $N$ is very important for these results. If boundary conditions were chosen so that $N$ had a non-trivial kernel, and if the flow profiles $U$ and $V$ were then chosen so that an element $y$ of that kernel also lay in the kernel of $M$, one would have $M y=N y=0$ and hence $M y=\lambda N y$ for any $\lambda \in \mathbb{C}$. Thus every $\lambda \in \mathbb{C}$ would be an eigenvalue.

\section{Open sets of eigenvalues}

The possible existence of open sets of eigenvalues will be encountered in our construction of the Titchmarsh-Weyl matrix $M(\lambda)$. It will also play an important role in the discussion of spectral inclusion and exactness. In the present section we shall show, at least for generic Reynolds numbers $R$, that the Ekman problem does not have an open set of eigenvalues. We have already shown (Theorem 5.3) that there is no open set of eigenvalues for the corresponding truncated problem. We begin with some examples of similar problems which do have open sets of eigenvalues.

Example 4. Consider the problem

$$
w^{\prime}=\lambda D w, \quad 0<x<\infty,
$$

where $w(x) \in \mathbb{C}^{6}, \quad \lambda \in \mathbb{C}, \quad D=\operatorname{diag}(-1,-2,-3,1,2,3)$, and with boundary conditions

$$
w_{4}(0)=w_{5}(0)=w_{6}(0)=0 .
$$

A value $\lambda$ is an eigenvalue if there exists a nontrivial solution $w(x, \lambda)=\left(w_{1}(x, \lambda)\right.$, $\left.w_{2}(x, \lambda), \ldots, w_{6}(x, \lambda)\right)^{T}$ with components $w_{k}(x, \lambda) \in L^{2}[0, \infty)$. The essential spectrum for this problem is the imaginary axis $\sigma_{\text {ess }}=\{\lambda \in \mathbb{C}: \operatorname{Re}(\lambda)=0\}$. The set of eigenvalues consists of all points in the right half-plane $\{\lambda \in \mathbb{C}: \operatorname{Re}(\lambda)>0\}$, and they all have multiplicity 3 . On the other hand, if we change the boundary conditions to

$$
w_{1}(0)-w_{4}(0)=w_{2}(0)-w_{5}(0)=w_{3}(0)-w_{6}(0)=0,
$$

then there are no eigenvalues. 
Example 5. Consider the corresponding truncated problem

$$
w^{\prime}=\lambda D w, \quad 0<x<1,
$$

with boundary conditions

$$
w_{1}(0)=w_{2}(0)=w_{3}(0)=0=w_{1}(1)=w_{2}(1)=w_{3}(1) .
$$

Then every $\lambda \in \mathbb{C}$ is a triple eigenvalue. On the other hand, if we change the boundary conditions to

$$
w_{1}(0)=w_{2}(0)=w_{3}(0)=0=w_{4}(1)=w_{5}(1)=w_{6}(1),
$$

then there are no eigenvalues.

We will now show that the perturbed Ekman problem does not have an open set of eigenvalues. Recall that this problem is

$$
\left(\begin{array}{l}
u^{\prime} \\
v^{\prime}
\end{array}\right)=A\left(\begin{array}{l}
u \\
v
\end{array}\right)
$$

with boundary conditions

$$
u_{1}(0)=u_{2}(0)=v_{1}(0)=0,
$$

where $A=A(\lambda)$ is given in (3.2). When $A$ has distinct eigenvalues, it can be diagonalized: $T^{-1} A T=D$, where $D=\operatorname{diag}\left(\mu_{1}, \mu_{2}, \ldots, \mu_{6}\right)$. If $\lambda \notin \sigma_{e s s}(M, N)$, then no eigenvalue of $A$ is purely imaginary. We shall always assume that these eigenvalues are ordered so that

$$
\operatorname{Re}\left(\mu_{1}\right), \operatorname{Re}\left(\mu_{2}\right), \operatorname{Re}\left(\mu_{3}\right)<0 \text { and } \operatorname{Re}\left(\mu_{4}\right), \operatorname{Re}\left(\mu_{5}\right), \operatorname{Re}\left(\mu_{6}\right)>0 .
$$

The column vector $t_{j}=\left(t_{1 j}, t_{2 j}, \ldots, t_{6 j}\right)^{T}$ of $T$ is an eigenvector of $A$ corresponding to $\mu_{j}$.

Representative eigenvectors of $A$ can be found from the block operator equation (1.1) by setting $y=e^{\mu x}, z=b e^{\mu x}$ and solving for $b$. The result is

$$
t_{i j}=p_{i}\left(\mu_{j}\right)
$$

where

$$
\begin{aligned}
& p_{1}(\mu)=\mu^{2}-\alpha^{2}+\lambda, \quad p_{2}(\mu)=\mu\left(\mu^{2}-\alpha^{2}+\lambda\right), \\
& p_{3}(\mu)=\mu^{2}\left(\mu^{2}-\alpha^{2}+\lambda\right), \quad p_{4}(\mu)=\mu^{3}\left(\mu^{2}-\alpha^{2}+\lambda\right), \\
& p_{5}(\mu)=2 \mu, \quad p_{6}(\mu)=2 \mu^{2} .
\end{aligned}
$$

As in (3.12), let

$$
T_{0}=\left(\begin{array}{ccc}
t_{11} & t_{12} & t_{13} \\
t_{21} & t_{22} & t_{23} \\
t_{51} & t_{52} & t_{53}
\end{array}\right)=\left(\begin{array}{lll}
p_{1}\left(\mu_{1}\right) & p_{1}\left(\mu_{2}\right) & p_{1}\left(\mu_{3}\right) \\
p_{2}\left(\mu_{1}\right) & p_{2}\left(\mu_{2}\right) & p_{2}\left(\mu_{3}\right) \\
p_{5}\left(\mu_{1}\right) & p_{5}\left(\mu_{2}\right) & p_{5}\left(\mu_{3}\right)
\end{array}\right) .
$$

Recall (Definition 3·1) that

$$
\Omega(M, N)=\mathbb{C} \backslash\left(\sigma_{\text {ess }}(M, N) \cup B\right),
$$

where $B$ is the set of branch points of the characteristic polynomial $p(\mu ; \lambda)$; and $\Omega(M, N)$ has two connected components $\Omega_{+}(M, N)$ and $\Omega_{-}(M, N)$, where $\Omega_{+}(M, N)$ 
is the component containing the interval $\left(\alpha^{2}, \infty\right) \subset \mathbb{R}$, and $\Omega_{-}(M, N)$ contains the interval $\left(-\infty, \alpha^{2}\right)$.

By Theorem $3 \cdot 5(3), \lambda$ is an eigenvalue for the problem $(6 \cdot 1)-(6 \cdot 2)$ if and only if $\operatorname{det}\left(T_{0}\right)=0$. We must show that $\operatorname{det}\left(T_{0}\right)$ does not have an open set of zeros. In fact, we shall show that $\operatorname{det}\left(T_{0}\right)$ has no zeros at all in $\Omega(M, N)$.

Lemma $6 \cdot 1$.

$$
\operatorname{det}\left(T_{0}\right)=2\left(\lambda-\alpha^{2}\right)\left(\mu_{1}-\mu_{2}\right)\left(\mu_{1}-\mu_{3}\right)\left(\mu_{2}-\mu_{3}\right) f(\lambda),
$$

where

$$
\begin{aligned}
& f(\lambda)=\alpha+\mu_{1}+\mu_{2}+\mu_{3}, \text { for } \lambda \in \Omega_{+}(M, N), \\
& f(\lambda)=-\alpha+\mu_{1}+\mu_{2}+\mu_{3}, \text { for } \lambda \in \Omega_{-}(M, N) .
\end{aligned}
$$

Proof. From $(6 \cdot 3)-(6 \cdot 5)$ and elementary manipulations of determinants, we obtain

$$
\operatorname{det}\left(T_{0}\right)=-2 \mu_{1} \mu_{2} \mu_{3}\left|\begin{array}{ccc}
\mu_{1}^{2} & \mu_{2}^{2} & \mu_{3}^{2} \\
\mu_{1} & \mu_{2} & \mu_{3} \\
1 & 1 & 1
\end{array}\right|+2\left(\lambda-\alpha^{2}\right)\left|\begin{array}{ccc}
\mu_{1}^{3} & \mu_{2}^{3} & \mu_{3}^{3} \\
\mu_{1} & \mu_{2} & \mu_{3} \\
1 & 1 & 1
\end{array}\right| .
$$

Furthermore,

$$
\left|\begin{array}{ccc}
\mu_{1}^{3} & \mu_{2}^{3} & \mu_{3}^{3} \\
\mu_{1} & \mu_{2} & \mu_{3} \\
1 & 1 & 1
\end{array}\right|=\left(\mu_{1}-\mu_{2}\right)\left(\mu_{1}-\mu_{3}\right)\left(\mu_{2}-\mu_{3}\right)\left(\mu_{1}+\mu_{2}+\mu_{3}\right)
$$

and

$$
\left|\begin{array}{ccc}
\mu_{1}^{2} & \mu_{2}^{2} & \mu_{3}^{2} \\
\mu_{1} & \mu_{2} & \mu_{3} \\
1 & 1 & 1
\end{array}\right|=\left(\mu_{1}-\mu_{2}\right)\left(\mu_{1}-\mu_{3}\right)\left(\mu_{2}-\mu_{3}\right)
$$

This implies that

$$
\operatorname{det}\left(T_{0}\right)=2\left(\mu_{1}-\mu_{2}\right)\left(\mu_{1}-\mu_{3}\right)\left(\mu_{2}-\mu_{3}\right) f(\lambda)
$$

where

$$
f(\lambda)=-\mu_{1} \mu_{2} \mu_{3}+\left(\lambda-\alpha^{2}\right)\left(\mu_{1}+\mu_{2}+\mu_{3}\right) .
$$

Now recall that $\mu_{j}(1 \leqslant j \leqslant 6)$ are zeros of

$$
\begin{aligned}
p(\mu) & =\operatorname{det}(A-\mu I)=\left(\mu^{2}-\alpha^{2}+\lambda\right)\left(\mu^{2}-\alpha^{2}\right)+4 \mu^{2} \\
& =\mu^{6}+\left(2 \lambda-3 \alpha^{2}\right) \mu^{4}+\left(\lambda^{2}-4 \alpha^{2} \lambda+3 \alpha^{4}+4\right) \mu^{2}-\alpha^{2}\left(\lambda-\alpha^{2}\right)^{2}
\end{aligned}
$$

and $\mu_{4}=-\mu_{1}, \mu_{5}=-\mu_{2}, \mu_{6}=-\mu_{3}$. This implies that

$$
\mu_{1}^{2} \mu_{2}^{2} \mu_{3}^{2}=\alpha^{2}\left(\lambda-\alpha^{2}\right)^{2}
$$

and so

$$
\mu_{1} \mu_{2} \mu_{3}= \pm \alpha\left(\lambda-\alpha^{2}\right)
$$

Note that $\mu_{1} \mu_{2} \mu_{3}$ is a single-valued analytic function in each connected component of $\Omega(M, N)$. For $\lambda \in \mathbb{R}, p(\mu)$ has real roots $\pm \mu_{1}$, and complex conjugate roots $\mu_{2}, \mu_{3}$. Therefore $\mu_{1} \mu_{2} \mu_{3} \in \mathbb{R}$ for $\lambda \in \mathbb{R}$. Since $\mu_{1}<0$, it follows that $\mu_{1} \mu_{2} \mu_{3}<0$ for $\lambda \in$ $\mathbb{R}, \lambda \neq \alpha^{2}$. Therefore $\mu_{1} \mu_{2} \mu_{3}=-\alpha\left(\lambda-\alpha^{2}\right)$ for $\lambda \in \Omega_{+}(M, N)$ and $\mu_{1} \mu_{2} \mu_{3}=\alpha\left(\lambda-\alpha^{2}\right)$ for $\lambda \in \Omega_{-}(M, N)$. Substituting this into $(6 \cdot 8)$, we obtain formulas $(6 \cdot 6)-(6 \cdot 7)$. 
Theorem $6 \cdot 1$. The perturbed Ekman problem $(6 \cdot 1)-(6 \cdot 2)$ does not have any eigenvalues in $\Omega(M, N)$.

Proof. We must show that det $\left(T_{0}\right)$ does not have any zeros in $\Omega(M, N)$. Equivalently, we will show that the function $f(\lambda)$ in $(6 \cdot 7)$ has no zeros in $\Omega(M, N)$. From $(6 \cdot 7)$ it is clear that $f(\lambda)$ has no zeros in $\Omega_{-}(M, N)$, since $\operatorname{Re}(f(\lambda))<0$ there. We now consider $\lambda \in \Omega_{+}(M, N)$.

Since the characteristic polynomial

$$
\begin{aligned}
p(\mu) & =\mu^{6}+\left(2 \lambda-3 \alpha^{2}\right) \mu^{4}+\left(\lambda^{2}-4 \alpha^{2} \lambda+3 \alpha^{4}+4\right) \mu^{2}-\alpha^{2}\left(\lambda-\alpha^{2}\right)^{2} \\
& =\left(\mu^{2}-\mu_{1}^{2}\right)\left(\mu^{2}-\mu_{2}^{2}\right)\left(\mu^{2}-\mu_{3}^{2}\right),
\end{aligned}
$$

it follows that

$$
\begin{aligned}
\mu_{1}^{2}+\mu_{2}^{2}+\mu_{3}^{2} & =3 \alpha^{2}-\lambda \\
\mu_{1}^{2} \mu_{2}^{2}+\mu_{1}^{2} \mu_{3}^{2}+\mu_{2}^{2} \mu_{3}^{2} & =\lambda^{2}-4 \alpha^{2} \lambda+3 \alpha^{4}+4, \\
\mu_{1}^{2} \mu_{2}^{2} \mu_{3}^{2} & =\alpha^{2}\left(\lambda-\alpha^{2}\right)^{2} .
\end{aligned}
$$

Therefore $\mu_{1} \mu_{2} \mu_{3}= \pm \alpha\left(\lambda-\alpha^{2}\right)$. As we noted in the proof of Lemma $6 \cdot 1, \mu_{1} \mu_{2} \mu_{3}=$ $-\alpha\left(\lambda-\alpha^{2}\right)$ for $\lambda \in \Omega_{+}(M, N)$. Suppose that $f(\lambda)=0$ for some $\lambda \in \Omega_{+}(M, N)$. Then

$$
\mu_{1}+\mu_{2}+\mu_{3}=-\alpha
$$

and

$$
\begin{aligned}
\mu_{1} \mu_{2} \mu_{3} & =-\alpha\left(\lambda-\alpha^{2}\right), \\
\left(\mu_{1} \mu_{2}+\mu_{1} \mu_{3}+\mu_{2} \mu_{3}\right)^{2} & =\left(\mu_{1}^{2} \mu_{2}^{2}+\mu_{1}^{2} \mu_{3}^{2}+\mu_{2}^{2} \mu_{3}^{2}\right)+2 \mu_{1} \mu_{2} \mu_{3}\left(\mu_{1}+\mu_{2}+\mu_{3}\right) \\
& =\lambda^{2}-4 \alpha^{2} \lambda+3 \alpha^{4}+4+2 \alpha^{2}\left(\lambda-\alpha^{2}\right) \\
& =\left(\lambda-\alpha^{2}\right)^{2}+4 .
\end{aligned}
$$

Therefore

$$
\mu_{1} \mu_{2}+\mu_{1} \mu_{3}+\mu_{2} \mu_{3}=\left[\left(\lambda-\alpha^{2}\right)^{2}+4\right]^{1 / 2},
$$

where $z^{1 / 2}$ indicates one of the square roots of $z$. It now follows that

$$
\left(\mu-\mu_{1}\right)\left(\mu-\mu_{2}\right)\left(\mu-\mu_{3}\right)=\mu^{3}+\alpha \mu^{2}+\left[\left(\lambda-\alpha^{2}\right)^{2}+4\right]^{1 / 2} \mu+\alpha\left(\lambda-\alpha^{2}\right),
$$

and

$$
\left(\mu+\mu_{1}\right)\left(\mu+\mu_{2}\right)\left(\mu+\mu_{3}\right)=\mu^{3}-\alpha \mu^{2}+\left[\left(\lambda-\alpha^{2}\right)^{2}+4\right]^{1 / 2} \mu-\alpha\left(\lambda-\alpha^{2}\right) .
$$

Consequently

$$
\begin{aligned}
p(\mu)= & \mu^{6}+\left(2 \lambda-3 \alpha^{2}\right) \mu^{4}+\left(\lambda^{2}-4 \alpha^{2} \lambda+3 \alpha^{4}+4\right) \mu^{2}-\alpha^{2}\left(\lambda-\alpha^{2}\right)^{2} \\
= & \left(\mu-\mu_{1}\right)\left(\mu-\mu_{2}\right)\left(\mu-\mu_{3}\right)\left(\mu+\mu_{1}\right)\left(\mu+\mu_{2}\right)\left(\mu+\mu_{3}\right) \\
= & \left(\mu^{3}+\alpha \mu^{2}+\left[\left(\lambda-\alpha^{2}\right)^{2}+4\right]^{1 / 2} \mu+\alpha\left(\lambda-\alpha^{2}\right)\right)\left(\mu^{3}-\alpha \mu^{2}\right. \\
& \left.+\left[\left(\lambda-\alpha^{2}\right)^{2}+4\right]^{1 / 2} \mu-\alpha\left(\lambda-\alpha^{2}\right)\right) \\
= & \mu^{6}+\left\{2\left[\left(\lambda-\alpha^{2}\right)^{2}+4\right]^{1 / 2}-\alpha^{2}\right\} \mu^{4}+\left(\lambda^{2}-4 \alpha^{2} \lambda+3 \alpha^{4}+4\right) \mu^{2}-\alpha^{2}\left(\lambda-\alpha^{2}\right)^{2} .
\end{aligned}
$$

Equating the coefficients of $\mu^{4}$, we obtain

$$
2\left[\left(\lambda-\alpha^{2}\right)^{2}+4\right]^{1 / 2}-\alpha^{2}=2 \lambda-3 \alpha^{2},
$$


which implies that $\left[\left(\lambda-\alpha^{2}\right)^{2}+4\right]^{1 / 2}=\lambda-\alpha^{2}$, and $\left(\lambda-\alpha^{2}\right)^{2}+4=\left(\lambda-\alpha^{2}\right)^{2}$. The Theorem follows from this contradiction.

We now consider the unperturbed Ekman problem in its guise as a system of first order ODEs $(2 \cdot 14)-(2 \cdot 16)$. For convenience we restate it here:

$$
\left(\begin{array}{l}
u^{\prime} \\
v^{\prime}
\end{array}\right)=G\left(\begin{array}{l}
u \\
v
\end{array}\right), \quad 0<x<\infty,
$$

with boundary conditions

$$
u_{1}(0)=u_{2}(0)=v_{1}(0)=0,
$$

where

$$
\begin{gathered}
G(x, \lambda)=A(\lambda)+Q(x), \\
Q(x)=\left(\begin{array}{cccccc}
0 & 0 & 0 & 0 & 0 & 0 \\
0 & 0 & 0 & 0 & 0 & 0 \\
0 & 0 & 0 & 0 & 0 & 0 \\
q_{41} & 0 & q_{43} & 0 & 0 & 0 \\
0 & 0 & 0 & 0 & 0 & 0 \\
q_{61} & 0 & 0 & 0 & q_{65} & 0
\end{array}\right), \\
q_{41}=-i R\left(\alpha^{3} V(x)+\alpha V^{\prime}(x)\right), \quad q_{43}=i \alpha R V(x), \\
q_{61}=i \alpha R U^{\prime}(x), \quad q_{65}=i \alpha R V(x) .
\end{gathered}
$$

Here, $R$ is the Reynolds number, and $A(\lambda)$ is given in (3.2) (and is the same matrix that appears in $(6 \cdot 1))$.

If $D$ is a disc contained in $\Omega(M, N)$, and $R_{0}>0$, Theorem $4 \cdot 1$ implies that there is a $6 \times 3$ matrix $Y_{\text {bound }}(x, \lambda, R)$ (defined for $0 \leqslant x<\infty, \lambda \in D, 0 \leqslant R \leqslant R_{0}$ ) with the following properties:

(1) the columns of $Y_{\text {bound }}$ span the space of $L^{2}$-solutions of (6.9);

(2) $Y_{\text {bound }}$ is an analytic function of $\lambda \in D$;

(3) $Y_{\text {bound }}$ is an analytic function of $R \in\left[0, R_{0}\right]$.

Note that property (3) follows from the proof of Theorem $4 \cdot 1$ for the same reason as (2), namely the contraction map $\Sigma$ is analytic in $R$.

Suppose that the columns of $Y_{\text {bound }}$ are $y_{i}(x, \lambda, R)=\left(y_{1 i}(x, \lambda, R), \ldots, y_{6 i}(x, \lambda, R)\right)^{T}$, for $1 \leqslant i \leqslant 3$. A value $\lambda \in D$ is an eigenvalue for the Ekman problem if and only if there exists $\left(c_{1}, c_{2}, c_{3}\right) \neq(0,0,0)$ such that $c_{1} y_{1}+c_{2} y_{2}+c_{3} y_{3}$ satisfies the boundary conditions $(6 \cdot 10)$, and this is equivalent to

$$
\operatorname{det} Y_{0}(\lambda, R)=0,
$$

where

$$
Y_{0}(\lambda, R)=\left(\begin{array}{lll}
y_{11}(0, \lambda, R) & y_{12}(0, \lambda, R) & y_{13}(0, \lambda, R) \\
y_{21}(0, \lambda, R) & y_{22}(0, \lambda, R) & y_{23}(0, \lambda, R) \\
y_{51}(0, \lambda, R) & y_{52}(0, \lambda, R) & y_{53}(0, \lambda, R)
\end{array}\right)
$$


Suppose that $D_{1}$ and $D_{2}$ are dises in $\Omega$, such that $D_{1} \cap D_{2} \neq \varnothing$. Let $Y_{01}(\lambda, R)$ (resp. $Y_{02}(\lambda, R)$ ) denote $Y_{0}(\lambda, R)$ in $D_{1}$ (resp. $\left.D_{2}\right)$. Then there is a $3 \times 3$ analytic matrix $C(\lambda, R)$ (defined for $\lambda \in D_{1} \cap D_{2}$ ) such that $\operatorname{det} C(\lambda, R) \neq 0$ and $Y_{02}(\lambda, R)=Y_{01}(\lambda, R) C(\lambda, R)$ for $\lambda \in D_{1} \cap D_{2}$. Thus $\operatorname{det} Y_{01}(\lambda, R)=0$ if and only if $\operatorname{det} Y_{02}(\lambda, R)=0$ (for $\left.\lambda \in D_{1} \cap D_{2}\right)$. If $\operatorname{det} Y_{01}(\lambda, R)=0$ for all $\lambda \in D_{1}$ (and some fixed $R$ ), then $\operatorname{det} Y_{02}(\lambda, R)=0$ for all $\lambda \in D_{2}$. By applying this fact to chains of intersecting discs, we see that if (for some given $R$ ) the Ekman problem has an open set of eigenvalues, then at least one of the connected components of $\Omega(M, N)$ consists entirely of eigenvalues. We can immediately show that this cannot happen in $\Omega_{-}(M, N)$.

Theorem 6.2. $\Omega_{-}(M, N)$ does not contain an open set of eigenvalues for the Ekman problem $(6 \cdot 9)-(6 \cdot 10)$.

Proof. As noted above, if there is an open set of eigenvalues in $\Omega_{-}(M, N)$, then this component consists entirely of eigenvalues. But by Theorem $5 \cdot 1$ the eigenvalues are located in a right half-plane.

Unfortunately, we are unable to prove this for the other component $\Omega_{+}(M, N)$. But we can show that for generic Reynolds numbers, $\Omega_{+}(M, N)$ does not have an open set of eigenvalues.

Theorem 6.3. Let $\mathfrak{R}_{O E}$ be the set of Reynolds numbers $\{R \in \mathbb{R}: R \geqslant 0\}$ such that the Ekman problem $(6 \cdot 9)-(6 \cdot 10)$ has a (nonempty) open set of eigenvalues.

(1) There exists $R_{0}>0$ such that $\left[0, R_{0}\right] \cap \mathfrak{R}_{O E}=\varnothing$.

(2) $\mathfrak{R}_{O E}$ has no accumulation points.

Proof. (1) The case $R=0$ corresponds to the perturbed Ekman problem (6.1)(6.2). For $R=0, Y_{0}(\lambda, R)=T_{0}(\lambda)$ (which was displayed in $(6 \cdot 5)$ ). By Theorem $6 \cdot 1$ $\operatorname{det} Y_{0}(\lambda, 0)=\operatorname{det} T_{0}(\lambda) \neq 0$, for all $\lambda \in \Omega(M, N)$. Let $\lambda_{0} \in \Omega_{+}(M, N)$. Since $Y_{0}(\lambda, R)$ is analytic (and hence continuous) in $R$, it follows that $\operatorname{det} Y_{0}\left(\lambda_{0}, R\right) \neq 0$ for small $R$.

(2) Suppose that there is a sequence $R_{n} \in \mathfrak{R}_{O E}$ such that $\lim _{n \rightarrow \infty} R_{n}=R_{0}$. Then for each $R_{n}$, the component $\Omega_{+}(M, N)$ consists entirely of eigenvalues. Let $\lambda_{0} \in \Omega_{+}(M, N)$; then $\operatorname{det} T_{0}\left(\lambda_{0}\right) \neq 0$. The function $f(R)=\operatorname{det} Y_{0}\left(\lambda_{0}, R\right)$ is an analytic function whose zeros accumulate. Therefore $f(R) \equiv 0$. But this contradicts our assumption that $f(0)=\operatorname{det} T_{0}\left(\lambda_{0}\right) \neq 0$.

\section{The Titchmarsh-Weyl coefficients}

We now know that $\Omega_{-}(M, N)$ contains no open set of eigenvalues for the Ekman problem, but there may be a sparse set $\Re_{O E}$ of Reynolds numbers for which $\Omega_{+}(M, N)$ consists entirely of eigenvalues. In this section we shall assume that the Reynolds number does not belong to this sparse set, which may exist.

$(\mathrm{A} 7 \cdot 1)$ We assume that the Reynolds number $R \notin \mathfrak{R}_{O E}$. Consequently, the Ekman problem does not have a (nonempty) open set of eigenvalues.

Since the eigenvalues are the zeros of a locally analytic function (the characteristic determinant $(6 \cdot 11)-(6 \cdot 12))$, assumption $(\mathrm{A} 7 \cdot 1)$ implies that the eigenvalues have no accumulation point in $\Omega(M, N)$. 
In Theorem $4 \cdot 1$ we proved that equation $(4 \cdot 1)$ has a $6 \times 3$ solution matrix $Y_{\text {bound }}(x, \lambda)$ in $L^{2}[0, \infty)$ with respect to $x$, and locally analytic in $\lambda$. In general, the analytic continuations of this matrix around branch points do not coincide with it. Thus $Y_{\text {bound }}(\cdot, \lambda)$ is really a multivalued function. We now consider the representation of this solution matrix in terms of solutions with Dirichlet or Neumann initial values. These solutions are single-valued entire functions. In order to agree with the accepted notation for self-adjoint Hamiltonian systems (see Hinton and Shaw [16]) we now let $\Phi$ and $\Theta$ denote the $6 \times 3$ solution matrices of $(4 \cdot 1)$ uniquely determined by the initial conditions

$$
\Phi(0, \lambda)=\left(\begin{array}{lll}
0 & 0 & 0 \\
0 & 0 & 0 \\
1 & 0 & 0 \\
0 & 1 & 0 \\
0 & 0 & 0 \\
0 & 0 & 1
\end{array}\right), \quad \Theta(0, \lambda)=\left(\begin{array}{lll}
1 & 0 & 0 \\
0 & 1 & 0 \\
0 & 0 & 0 \\
0 & 0 & 0 \\
0 & 0 & 1 \\
0 & 0 & 0
\end{array}\right)
$$

The columns of $\Phi$ span the set of all solutions of $(4 \cdot 1)$ which satisfy the boundary conditions at $x=0$, while the columns of $\Theta$ span the remainder of the solution space. Thus the $L^{2}$ solution matrix $Y_{\text {bound }}$ of Theorem $4 \cdot 1$ must be a linear combination of $\Theta$ and $\Phi$ :

$$
Y_{\text {bound }}(x, \lambda)=\Theta(x, \lambda) C_{1}(\lambda)+\Phi(x, \lambda) C_{2}(\lambda),
$$

for some $3 \times 3$ matrices $C_{1}$ and $C_{2}$. Now $\lambda$ is an eigenvalue of the Ekman problem if and only if it has an associated eigenfunction. In order to lie in $L^{2}[0, \infty)$ this eigenfunction must be a linear combination of the columns of $Y_{\text {bound }}(x, \lambda)$; in order to satisfy the boundary conditions at $x=0$, it must be a linear combination of columns of $\Phi$. Thus $\lambda$ is an eigenvalue if and only if there exists a linear combination of columns of $Y_{\text {bound }}(x, \lambda)$ which is a linear combination of columns of $\Phi(x, \lambda)$. Since $\Theta$ is a full rank matrix this will happen if and only if $C_{1}(\lambda)$ has a non-trivial null space, proving the following:

Lemma 7·1. $\lambda$ is an eigenvalue of the Ekman problem if and only if $\operatorname{det}\left(C_{1}(\lambda)\right)=0$.

When $\lambda$ is not an eigenvalue, then, $C_{1}(\lambda)$ will be invertible, and so the matrix

$$
\Psi(x, \lambda):=Y_{\text {bound }}(x, \lambda) C_{1}(\lambda)^{-1}=\Theta(x, \lambda)+\Phi(x, \lambda) M(\lambda), M(\lambda):=C_{2}(\lambda) C_{1}(\lambda)^{-1},
$$

will be a well-defined solution matrix of $(4 \cdot 1)$ whose columns span the set of all $L^{2}$ solutions of $(4 \cdot 1)$.

Equation $(7 \cdot 2)$ can be written in the form

$$
Y_{\text {bound }}(\cdot, \lambda)=(\Theta(\cdot, \lambda) \Phi(\cdot, \lambda))\left(\begin{array}{l}
C_{1}(\lambda) \\
C_{2}(\lambda)
\end{array}\right)
$$

The columns of $(\Theta(\cdot, \lambda) \Phi(\cdot, \lambda))$ are linearly independent, and so (7.4) can be solved for $C_{1}(\lambda)$ and $C_{2}(\lambda)$ by Cramer's rule. Since $Y_{\text {bound }}(\cdot, \lambda)$ is locally analytic from the results in Section 4 , and $\Theta(\cdot, \lambda), \Phi(\cdot, \lambda)$ are entire functions, it follows from the local analyticity of $Y_{\text {bound }}$ that $C_{1}(\lambda)$ and $C_{2}(\lambda)$ are locally analytic. Thus the matrix $M(\lambda)$ appearing in $(7 \cdot 3)$ is locally analytic wherever $C_{1}(\lambda)$ is invertible. By assumption 
(A7.1), $\operatorname{det}\left(C_{1}(\lambda)\right)$ cannot be zero on an open set. Therefore $M(\lambda)$ is meromorphic on any disc $D \subset \Omega(M, N)$. In fact, $M(\lambda)$ and $\Psi(\cdot, \lambda)$ are single-valued.

Theorem 7.1. $M(\lambda)$ and $\Psi(\cdot, \lambda)$ are well-defined, single-valued meromorphic matrix functions on $\Omega(M, N)$.

Proof. Suppose that $Y_{\text {bound }}(x, \lambda)$ and $\hat{Y}_{\text {bound }}(x, \lambda)$ are $6 \times 3$ analytic solution matrices defined on some dise $D \subset \Omega(M, N)$, such that the columns of each matrix span the space of $L^{2}$-solutions of $(4 \cdot 1)$. Then there is a $3 \times 3$ invertible, analytic matrix $B(\lambda)$ such that $\hat{Y}_{\text {bound }}(x, \lambda)=Y_{\text {bound }}(x, \lambda) B(\lambda)$. We now have:

$$
\begin{aligned}
& Y_{\text {bound }}(x, \lambda)=\Theta(x, \lambda) C_{1}(\lambda)+\Phi(x, \lambda) C_{2}(\lambda), \\
& \hat{Y}_{\text {bound }}(x, \lambda)=\Theta(x, \lambda) \hat{C}_{1}(\lambda)+\Phi(x, \lambda) \hat{C}_{2}(\lambda)
\end{aligned}
$$

and

$$
\hat{Y}_{\text {bound }}(x, \lambda)=Y_{\text {bound }}(x, \lambda) B(\lambda)=\Theta(x, \lambda) C_{1}(\lambda) B(\lambda)+\Phi(x, \lambda) C_{2}(\lambda) B(\lambda) .
$$

Therefore $\hat{C}_{1}=C_{1} B$ and $\hat{C}_{2}=C_{2} B$, so that

$$
\hat{\Psi}=\hat{Y}_{\text {bound }} \hat{C}_{1}^{-1}=Y_{\text {bound }} B\left(C_{1} B\right)^{-1}=Y_{\text {bound }} C_{1}^{-1}=\Psi
$$

and

$$
\hat{M}=\hat{C}_{2} \hat{C} 1^{-1}=C_{2} B\left(C_{1} B\right)^{-1}=C_{2} C_{1}^{-1}=M .
$$

This proves the Theorem.

Definition $7 \cdot 1$. We call the matrix $M(\lambda)$ appearing in $(7 \cdot 3)$ the Titchmarsh-Weyl matrix for the system $(4 \cdot 1)$.

There is a well developed theory of Titchmarsh-Weyl matrices for selfadjoint Hamiltonian systems: see [16]. In particular it is known that $M(\lambda)$ is analytic in the whole of $\mathbb{C} \backslash \mathbb{R}$ and that it has a pole at any isolated eigenvalue, with an essential singularity at any point of the essential spectrum. For non-selfadjoint systems none of this theory exists, in general: we rely heavily on the Levinson asymptotics, and in particular on Theorem $4 \cdot 1$, to guarantee existence and analyticity of $M(\lambda)$ here. However the following remains true.

Theorem 7.2. A point $\mu \in \Omega(M, N)$ is an eigenvalue of the Ekman problem if and only if $\mu$ is a pole of $M(\lambda)$.

Proof. Suppose that $\lambda=\mu$ is an eigenvalue of the problem. Then the matrix $C_{1}(\lambda)$ is rank deficient at $\lambda=\mu$. This means that its determinant has a zero at $\mu$. If this zero were not of finite multiplicity then, since $\operatorname{det} C_{1}(\lambda)$ is analytic at $\mu$, $\operatorname{det} C_{1}(\lambda)$ would be identically zero, contradicting assumption (A7-1). Thus $\operatorname{det} C_{1}(\lambda)$ has a zero of finite multiplicity at $\mu$, and so $M(\lambda)$ has (at worst) a pole of finite multiplicity at $\mu$. We now show that this pole is non-trivial. Choose a vector $w$ such that $Y_{\text {bound }}(x, \mu) w$ is an eigenfunction belonging to the eigenvalue $\mu$, and observe that since this eigenfunction satisfies the boundary conditions at $x=0$,

$$
Y_{\text {bound }}(x, \mu) w=\Phi(x, \mu) C_{2}(\mu) w ;
$$

in particular, this means that $C_{2}(\mu) w \neq \mathbf{0}$ and $C_{1}(\mu) w=\mathbf{0}$. This means that $w \neq \mathbf{0}$ and 
$C_{1}(\mu) w=\mathbf{0}$. Define $v(\lambda)=C_{1}(\lambda) w$ so that $v(\mu)=\mathbf{0}$; since $\mu$ is an isolated eigenvalue of the problem, $v(\lambda) \neq \mathbf{0}$ for $\lambda$ in a punctured neighbourhood of $\mu$. Now observe that

$$
C_{2}(\lambda) w=M(\lambda) v(\lambda) .
$$

Since $C_{2}(\mu) w \neq \mathbf{0}$ and $v(\mu)=\mathbf{0}$ it follows that $M$ has a non-trivial pole at $\mu$.

Now suppose that $M(\lambda)$ has a pole at $\lambda=\mu$. Choose a vector $v(\lambda)$ (not necessarily as an analytic function of $\lambda$ ) such that $w(\lambda)=M(\lambda) v(\lambda)$ has the property $\|w(\lambda)\|=1$ $(\lambda \neq \mu)$ and such that $\lim _{\lambda \rightarrow \mu} v(\lambda)=\mathbf{0}$. Observe that

$$
w(\lambda)=M(\lambda) v(\lambda)=C_{2}(\lambda) z(\lambda), \text { where } z(\lambda):=C_{1}(\lambda)^{-1} v(\lambda) .
$$

Observe also that

$$
Y_{\text {bound }}(x, \lambda) z(\lambda)=\Theta(x, \lambda) v(\lambda)+\Phi(x, \lambda) w(\lambda) .
$$

Since the vector $w(\lambda)$ lies on the unit sphere, by compactness we can choose a sequence $\left(\lambda^{(p)}\right)_{p \in \mathbb{N}}$ with limit $\mu$ such that $w_{\mu}:=\lim _{p \rightarrow \infty} w\left(\lambda^{(p)}\right)$ exists and is of unit length. We already know that $\lim _{\lambda \rightarrow \mu} v(\lambda)=\mathbf{0}$. Hence from $(7 \cdot 6)$, as the matrix $Y_{\text {bound }}(x, \mu)$ is of full rank, the limit $z_{\mu}:=\lim _{p \rightarrow \infty} z\left(\lambda^{(p)}\right)$ exists and is non-zero. Moreover,

$$
Y_{\text {bound }}(x, \mu) z_{\mu}=\Phi(x, \mu) w_{\mu} \text {. }
$$

Thus $\mu$ is an eigenvalue with eigenfunction $Y_{\text {bound }}(\cdot, \mu) z_{\mu}$.

Consider now a problem for $(4 \cdot 1)$ posed on an interval $[0, X]$, with some regular boundary conditions at $x=X$ which do not depend on $\lambda$. Writing a solution vector $Y=\left(Y_{1}, \ldots, Y_{6}\right)^{T}$, the boundary conditions might be, for example, the same as the boundary conditions imposed at $x=0$,

$$
Y_{1}(X)=Y_{2}(X)=Y_{5}(X)=0 .
$$

There will exist a $6 \times 3$ solution matrix $Y_{X}(x, \lambda)$ whose columns span the space of solutions satisfying the boundary condition at $x=X$. This time, standard theory of regular initial value problems guarantees that $Y_{X}$ can be chosen as an entire function of $\lambda$. Just as we obtained a matrix $\Psi$ given in (7.3) from the matrix $Y_{\text {bound }}$ of $L^{2}$ solutions in the infinite interval case, so now it will be possible to obtain from $Y_{X}(x, \lambda)$ a matrix $\Psi_{X}(x, \lambda)$ of the form

$$
\Psi_{X}(x, \lambda)=\Theta(x, \lambda)+\Phi(x, \lambda) M_{X}(\lambda)
$$

whose columns will span the space of all solutions satisfying the boundary conditions at $x=X$, provided $\lambda$ is not an eigenvalue of the problem on $[0, X]$. We shall call the matrix $M_{X}(\lambda)$ the Titchmarsh-Weyl matrix for the problem on the truncated interval $[0, X]$. The proof of the following result is very similar to the proof of Theorem $7 \cdot 2$.

Theorem 7.3. A point $\mu \in \mathbb{C}$ is an eigenvalue of the finite interval problem over $[0, X]$ if and only if $\mu$ is a pole of $M_{X}(\lambda)$.

Note that Theorem 5.3 says that the eigenvalues of the truncated problem have no accumulation points in $\mathbb{C}$. Thus $M_{X}(\lambda)$ is a meromorphic matrix function on $\mathbb{C}$. The obvious question is whether or not we have

$$
\lim _{X \rightarrow \infty} M_{X}(\lambda)=M(\lambda)
$$


at least for those $\lambda$ where $M(\lambda)$ is analytic. To examine this question we proceed as follows.

Firstly, assume that the boundary condition at $x=X$ is indeed (7.7). This means that $\Psi_{X}(X, \lambda)$ has the form

$$
\Psi_{X}(X, \lambda)=\left(\begin{array}{ccc}
0 & 0 & 0 \\
0 & 0 & 0 \\
* & * & * \\
* & * & * \\
0 & 0 & 0 \\
* & * & *
\end{array}\right) .
$$

Introduce the $6 \times 6$ permutation matrix $P_{35}$ given by

$$
P_{35}=\text { matrix obtained by swapping rows } 3 \text { and } 5 \text { of the identity. }
$$

(For different boundary conditions at $x=X$, a different matrix from $P_{35}$ will be required, but the arguments below are otherwise unchanged.) Thus $P_{35} \Psi_{X}(X, \lambda)$ has the form

$$
P_{35} \Psi_{X}(X, \lambda)=\left(\begin{array}{ccc}
0 & 0 & 0 \\
0 & 0 & 0 \\
0 & 0 & 0 \\
* & * & * \\
* & * & * \\
* & * & *
\end{array}\right),
$$

in which the lower $3 \times 3$ block is invertible. However, we also know from $(7 \cdot 8)$ that

$$
P_{35} \Psi_{X}(X, \lambda)=P_{35} \Theta(X, \lambda)+P_{35} \Phi(X, \lambda) M_{X}(\lambda) .
$$

Taking the top $3 \times 3$ blocks on both sides of this equation yields zero on the left hand side and thus gives

$$
\left(P_{35} \Phi(X, \lambda)\right)_{T O P} M_{X}(\lambda)=-\left(P_{35} \Theta(X, \lambda)\right)_{T O P},
$$

in which the subscript 'TOP' attached to a $6 \times 3$ matrix denotes the top $3 \times 3$ block of that matrix.

Lemma 7.2. Suppose that $\lambda_{0} \in \Omega(M, N)$ is not an eigenvalue of the Ekman problem. Then there exists a disc $D \subset \Omega(M, N)$ centered at $\lambda_{0}$ and $X_{0}>0$ such that $\left(P_{35} \Phi(x, \lambda)\right)_{T O P}$ is invertible for all $\lambda \in D$ and $x \geqslant X_{0}$.

Proof. First assume that the real parts of $\mu_{j}\left(\lambda_{0}\right)$ are distinct, and the $\mu_{j}$ are ordered so that

$$
\operatorname{Re}\left(\mu_{4}\left(\lambda_{0}\right)\right)<\operatorname{Re}\left(\mu_{5}\left(\lambda_{0}\right)\right)<\operatorname{Re}\left(\mu_{6}\left(\lambda_{0}\right)\right) .
$$

Then $\lambda_{0}$ has a neighbourhood $D \subset \Omega(M, N)$ which contains no eigenvalues and such that

$$
\operatorname{Re}\left(\mu_{4}(\lambda)\right)<\operatorname{Re}\left(\mu_{5}(\lambda)\right)<\operatorname{Re}\left(\mu_{6}(\lambda)\right) \text { for } \lambda \in D .
$$

Let $Y_{\text {unbound }}$ be the $6 \times 3$ full rank solution matrix whose columns are the solutions whose existence is guaranteed by Theorem $4 \cdot 2$. We can express $\Phi(x, \lambda)$ in the form

$$
\Phi(x, \lambda)=Y_{\text {bound }}(x, \lambda) B_{1}(\lambda)+Y_{\text {unbound }}(x, \lambda) B_{2}(\lambda),
$$


for some $3 \times 3$ locally analytic matrices $B_{1}(\lambda)$ and $B_{2}(\lambda)$. If $B_{2}(\lambda)$ has a non-trivial null-space then $\lambda$ is clearly an eigenvalue. Thus when $\lambda$ is not an eigenvalue, $B_{2}(\lambda)$ is invertible. The asymptotics of Theorems $4 \cdot 1$ and $4 \cdot 2$ then make it clear that for large $x$,

$$
\Phi(x, \lambda) \sim Y_{\text {unbound }}(x, \lambda) B_{2}(\lambda) .
$$

It now follows from Theorem $4 \cdot 2$ that

$$
\begin{aligned}
\left(P_{35} \Phi(x, \lambda)\right)_{T O P} & \sim\left(P_{35} Y_{\text {unbound }}\right)_{T O P} B_{2}(\lambda) \sim\left(\begin{array}{lll}
v_{14} & v_{15} & v_{16} \\
v_{24} & v_{25} & v_{26} \\
v_{54} & v_{55} & v_{56}
\end{array}\right) B_{2}(\lambda) \\
& =\left(\begin{array}{lll}
t_{14} & t_{15} & t_{16} \\
t_{24} & t_{25} & t_{26} \\
t_{54} & t_{55} & t_{56}
\end{array}\right) B_{2}(\lambda)=: T_{1} B_{2}(\lambda)
\end{aligned}
$$

uniformly for $\lambda \in D$ as $x \rightarrow \infty$. Here, $v_{j}=\left(v_{1 j}, v_{2 j}, \cdots, v_{6 j}\right)^{T}, 4 \leqslant j \leqslant 6$ are the vectors in Theorem 4.2, and the elements $t_{i j}$ in the matrices $T_{1}, T_{0}$ and $T$ are given in $(6 \cdot 3)$.

By $(6 \cdot 3)-(6 \cdot 4)$ we see that $\operatorname{det}\left(T_{1}\right)$ is obtained from $\operatorname{det}\left(T_{0}\right)$ by replacing $\left(\mu_{1}, \mu_{2}, \mu_{3}\right)$ by $\left(\mu_{4}, \mu_{5}, \mu_{6}\right)$. Thus, by Lemma $6 \cdot 1$

$$
\operatorname{det}\left(T_{1}\right)=2\left(\lambda-\alpha^{2}\right)\left(\mu_{4}-\mu_{5}\right)\left(\mu_{4}-\mu_{6}\right)\left(\mu_{5}-\mu_{6}\right) g(\lambda),
$$

where

$$
\begin{aligned}
& g(\lambda)=\alpha+\mu_{4}+\mu_{5}+\mu_{6}=-\left(-\alpha+\mu_{1}+\mu_{2}+\mu_{3}\right) \text { for } \lambda \in \Omega_{+}(M, N), \\
& g(\lambda)=-\alpha+\mu_{4}+\mu_{5}+\mu_{6}=-\left(\alpha+\mu_{1}+\mu_{2}+\mu_{3}\right) \text { for } \lambda \in \Omega_{-}(M, N),
\end{aligned}
$$

$g(\lambda)$ has no zeros in $\Omega_{+}(M, N)$. since $\operatorname{Re}(g(\lambda))>0$ there. By following the proof of Theorem $6 \cdot 1$ we find that $(7 \cdot 13)$ leads to one possible zero in $\Omega_{-}(M, N)$, namely $\lambda=\alpha^{2}+4 \alpha^{-2}$, but this lies in $\Omega_{+}(M, N)$. Therefore $\operatorname{det}\left(T_{1}(\lambda)\right) \neq 0$ for $\lambda \in \Omega(M, N)$. This proves the Lemma in case the real parts of the $\mu_{j}\left(\lambda_{0}\right)$ are distinct.

Let $\nu_{j}\left(\lambda_{0}\right)=\operatorname{Re}\left(\mu_{j}\left(\lambda_{0}\right)\right)$, and suppose, for example, that $\nu_{4}\left(\lambda_{0}\right)=\nu_{5}\left(\lambda_{0}\right)<\nu_{6}\left(\lambda_{0}\right)$. Then let $D$ be a neighbourhood of $\lambda_{0}$ containing no eigenvalues, and such that $\nu_{4}(\lambda)<\nu_{6}(\lambda)$ and $\nu_{5}(\lambda)<\nu_{6}(\lambda)$. Let $D_{1}=\left\{\lambda \in D: \nu_{4}(\lambda) \leqslant \nu_{5}(\lambda)\right\}$, and $D_{2}=\{\lambda \in$ $\left.D: \nu_{4}(\lambda) \geqslant \nu_{5}(\lambda)\right\}$. Apply Theorem $4 \cdot 2$ separately to $D_{1}$ and $D_{2}$. If $\nu_{4}\left(\lambda_{0}\right)=\nu_{5}\left(\lambda_{0}\right)=$ $\nu_{6}\left(\lambda_{0}\right)$, then choose a neighbourhood $D$ of $\lambda_{0}$ containing no eigenvalues, and divide it into six subsets, corresponding to the six order relations among $\nu_{1}, \nu_{2}$ and $\nu_{3}$. Theorem $4 \cdot 2$ can be applied to each of these subsets.

Now (for sufficiently large $X$ ) we can solve for $M_{X}(\lambda)$ in $(7 \cdot 10)$ :

$$
M_{X}(\lambda)=-\left(P_{35} \Phi(X, \lambda)\right)_{T O P}^{-1}\left(P_{35} \Theta(X, \lambda)\right)_{T O P} .
$$

The expression (7.14) is valid whenever $\lambda$ is not an eigenvalue of the problem on the finite interval $[0, X]$. Next, we introduce $M(\lambda)$ by using $(7 \cdot 3)$ in the form

$$
\Theta(X, \lambda)=\Psi(X, \lambda)-\Phi(X, \lambda) M(\lambda) .
$$

Substituting this expression for $\Theta(X, \lambda)$ into $(7 \cdot 14)$ yields

$$
M_{X}(\lambda)=M(\lambda)-\left(P_{35} \Phi(X, \lambda)\right)_{T O P}^{-1}\left(P_{35} \Psi(X, \lambda)\right)_{T O P} .
$$


Lemma 7.3. If $\lambda_{0} \in \Omega(M, N)$ is not a pole of $M(\lambda)$, then there is a neighbourhood $D$ of $\lambda_{0}$ and $X_{0}>0$ such that $M_{X}(\lambda)$ has no pole in $D$ for $X>X_{0}$.

Proof. By Theorem 7.2, $\lambda_{0}$ is not an eigenvalue of the Ekman problem. By Lemma $7 \cdot 2$ there is a neighbourhood $D$ of $\lambda_{0}$ and $X_{0}>0$ such that $\left(P_{35} \Phi(x, \lambda)\right)_{T O P}$ is invertible for all $\lambda \in D$ and $x \geqslant X_{0}$. We may suppose that $D$ contains no poles of $M(\lambda)$. Equation $(7 \cdot 15)$ is valid at all points $\lambda \in D_{0}$ where $M_{X}(\lambda)$ does not have a pole. Since the right-hand side of $(7 \cdot 15)$ is analytic in $D, M_{X}(\lambda)$ does not have any poles there.

Lemma 7.4. Suppose that $\lambda_{0} \in \Omega(M, N)$ is not a pole of $M(\lambda)$. Then there exists a disc $D \subset \Omega(M, N)$ centered at $\lambda_{0}$ such that

$$
\lim _{X \rightarrow \infty} M_{X}(\lambda)=M(\lambda)
$$

uniformly for $\lambda \in D$.

Proof. Theorem 7.2 implies that $\lambda_{0}$ is not an eigenvalue of the Ekman problem. Using the same strategy as in the proof of Lemma $7 \cdot 2$, we first consider the case where

$$
\operatorname{Re}\left(\mu_{4}\left(\lambda_{0}\right)<\operatorname{Re}\left(\mu_{5}\left(\lambda_{0}\right)<\operatorname{Re}\left(\mu_{6}\left(\lambda_{0}\right) .\right.\right.\right.
$$

Substituting $(7 \cdot 11)$ into $(7 \cdot 15)$, we obtain

$$
M_{X}(\lambda)-M(\lambda) \sim-\left(P_{35} Y_{\text {unbound }}(X, \lambda) B_{2}\right)_{T O P}^{-1}\left(P_{35} \Psi(X, \lambda)\right)_{T O P} .
$$

The right-hand side of $(7 \cdot 16)$ tends to zero uniformly in $D$ as $X \rightarrow \infty$. This proves the Theorem in case the $\mu_{j}\left(\lambda_{0}\right)$ have distinct real parts. If any of the real parts are equal, we subdivide $D$ into two or six subsets, as in the proof of Lemma $7 \cdot 2$. The convergence is uniform on each of these (finitely many) subsets, and therefore it is uniform on $D$.

Theorem 7.4. Let $\mathcal{K} \subset \Omega(M, N)$ be a compact set containing no poles of $M(\lambda)$. Then

$$
\lim _{X \rightarrow \infty} M_{X}(\lambda)=M(\lambda)
$$

uniformly for $\lambda \in \mathcal{K}$.

Proof. By Lemma $7 \cdot 4$ each point $\mu \in \mathcal{K}$ has a neigborhood $D_{\mu}$ such that $\lim _{X \rightarrow \infty} M_{X}(\lambda)=M(\lambda)$ uniformly for $\lambda \in D_{\mu}$. Since $\mathcal{K}$ is compact, it is covered by finitely many of these neighbourhoods, and so the convergence is uniform in $\mathcal{K}$.

\section{Spectral inclusion and spectral exactness}

Assumption (A7-1) will continue to be in force in this section. Thus the Ekman problem does not have an open set of eigenvalues. Consequently, the TitchmarshWeyl matrix $M(\lambda)$ is meromorphic on $\Omega(M, N)$, while $M_{X}(\lambda)$ is meromorphic on $\mathbb{C}$.

We now examine the effects on the spectrum of replacing the infinite interval $[0, \infty)$ by a finite interval $[0, X]$. To this end it is convenient to introduce some notation and terminology concerning eigenvalues.

Definition 8.1. Let $L_{\infty}(\lambda)$ denote the operator $L=M-\lambda N$ on $[0, \infty)$ with boundary conditions at $x=0$; and let $L_{X}(\lambda)$ denote the operator $L$ on the interval $[0, X]$ 
with boundary conditions at $x=0, X$. We shall say that $\lambda$ is an eigenvalue for $(M, N ; \infty)(\operatorname{resp} .(M, N ; X))$ if $L_{\infty}(\lambda)\left(\operatorname{resp} . L_{X}(\lambda)\right)$ is not injective. Let $\sigma_{p}(M, N ; \infty)$ $\left(\right.$ resp. $\left.\sigma_{p}(M, N ; X)\right)$ denote the set of eigenvalues for $(M, N ; \infty)(\operatorname{resp} .(M, N ; X))$ in $\Omega(M, N)($ resp. $\mathbb{C})$.

Assumption $(\mathrm{A} 7 \cdot 1)$ implies that $\sigma_{p}(M, N, \infty)$ has no accumulation points in $\Omega(M, N)$, while $\sigma_{p}(M, N, X)$ has no accumulation points in $\mathbb{C}$.

Definition 8.2. Let $\left\{X_{n}: n \in \mathbb{N}\right\}$ be a sequence in $(0, \infty)$ such that $\lim _{n \rightarrow \infty} X_{n}=\infty$.

(i) The sequence $\left\{\left(M, N ; X_{n}\right): n \in \mathbb{N}\right\}$ is spectrally inclusive for $(M, N ; \infty)$ if for every $\lambda_{0} \in \sigma_{p}(M, N ; \infty)$ there is a sequence $\left\{\lambda_{n}: n \in \mathbb{N}\right\}$ such that $\lambda_{n} \in \sigma_{p}\left(M, N ; X_{n}\right)$ and $\lim _{n \rightarrow \infty} \lambda_{n}=\lambda_{0}$.

(ii) The sequence $\left\{\left(M, N ; X_{n}\right): n \in \mathbb{N}\right\}$ is spectrally exact for $(M, N ; \infty)$ if it is spectrally inclusive and if whenever $\left\{\lambda_{n}: n \in \mathbb{N}\right\}$ is a sequence such that $\lambda_{n} \in \sigma_{p}\left(M, N ; X_{n}\right)$ and $\lim _{n \rightarrow \infty} \lambda_{n}=\lambda_{0} \in \Omega(M, N)$, then $\lambda_{0} \in \sigma_{p}(M, N ; \infty)$.

Remark 4. The above definition is a modification of the standard definition, which includes the entire spectrum, not just the point spectrum. (cf. $[\mathbf{5}, \mathbf{6 ,} 23,25,26]$.)

Theorem 8.1. For any sequence $\left\{X_{n}: n \in \mathbb{N}\right\}$ in $(0, \infty)$ such that $\lim _{n \rightarrow \infty} X_{n}=\infty$, $\left\{\left(M, N ; X_{n}\right): n \in \mathbb{N}\right\}$ is spectrally exact for $(M, N ; \infty)$.

Proof. We first show that $\left\{\left(M, N ; X_{n}\right): n \in \mathbb{N}\right\}$ is spectrally inclusive for $(M, N ; \infty)$. Let $\lambda_{0} \in \sigma_{p}(M, N ; \infty)$. Then $M(\lambda)=\left(m_{i j}(\lambda)\right)$ has a pole at $\lambda_{0}$. Therefore some coefficient $m_{i j}(\lambda)$ has a pole of order $k \geqslant 1$ at $\lambda_{0}$. Let $\epsilon>0$ be small enough so that $m_{i j}(\lambda)$ is analytic in the punctured disc $U_{\epsilon}=\left\{\lambda \in \mathbb{C}: 0<\left|\lambda-\lambda_{0}\right| \leqslant \epsilon\right\}$ and has no zeros there. Then, by the argument principle,

$$
\int_{C_{\epsilon}} \frac{m_{i j}^{\prime}(\lambda)}{m_{i j}(\lambda)} d \lambda=-k,
$$

where $C_{\epsilon}=\left\{\lambda:\left|\lambda-\lambda_{0}\right|=\epsilon\right\}$. Let $M_{X}(\lambda)=\left(m_{i j}(\lambda ; X)\right)$. By Theorem $7 \cdot 4, m_{i j}\left(\lambda ; X_{n}\right) \rightarrow$ $m_{i j}(\lambda)$ uniformly on the circle $C_{\epsilon}$. Therefore, for sufficiently large $n$,

$$
\int_{C_{\epsilon}} \frac{m_{i j}^{\prime}(\lambda ; X)}{m_{i j}(\lambda ; X)} d \lambda=-k,
$$

and so there is an eigenvalue $\lambda_{n}$ for $(M, N ; X)$ in $U_{\epsilon}$. This shows that the sequence is spectrally inclusive.

The spectral exactness is an immediate consequence of Lemma $7 \cdot 3$.

\section{Compound matrices and shooting}

\subsection{Transformation to compound matrices}

We have already seen in Section 2 that there is a class of block operator problems which can be reduced to systems of ordinary differential equations with (usually) $\lambda$-rational coefficients. In the case of the Ekman problem the system is $\lambda$-linear.

The Ekman problem involves first-order derivatives and so it is not possible to reduce it to a system of the form $Y^{\prime \prime}=A(x, \lambda) Y$ for which, as we showed in [14], a particularly efficient numerical approach is available. Fortunately it can be reduced to a system of the form $Y^{\prime}=A(x, \lambda) Y$ in which $A$ is only $6 \times 6$, as we showed in Section 2. Thus the use of compound matrices is still feasible. 
The compound matrix method can be traced back at least as far as the 1966 paper of Gilbert and Backus [13] and has been developed by a number of authors including Davies [8] and Ng and Reid [22]. For a recent description and analysis of the compound matrix method in terms of exterior algebras see Allen and Bridges [3]. In this very short section, included for completeness, we describe the compound matrix system which arises for the Ekman problem. The numerical results which follow from using this formulation are presented in Section 10.

Let $Y_{a}$ be a 'minimal fundamental' matrix spanning the space of all solutions of $Y^{\prime}=A(x, \lambda) Y$ which satisfy the boundary conditions at (say) $x=a$. Since $A$ is $6 \times 6$ and since there are 3 boundary conditions at $x=a, Y_{a}$ will be a $6 \times 3$ matrix. We partition $Y_{a}$ as $Y_{a}=\left(\begin{array}{l}U \\ V\end{array}\right)$. The fundamental matrix variable $z$ is a vector-valued function of $(x, \lambda)$ with values in $\mathbb{C}^{20}$. The order of its entries is arbitrary, but we chose the ordering

$z=\left(z_{1}, z_{2}, \phi_{11}, \phi_{12}, \phi_{13}, \phi_{21}, \phi_{22}, \phi_{23}, \phi_{31}, \phi_{32}, \phi_{33}, \psi_{11}, \psi_{12}, \psi_{13}, \psi_{21}, \psi_{22}, \psi_{23}, \psi_{31}, \psi_{32}, \psi_{33}\right)^{T}$

in which

$$
\begin{aligned}
& \phi_{i j}=\text { the determinant obtained by replacing row } j \text { in } U \text { by row } i \text { in } V, \\
& \psi_{i j}=\text { the determinant obtained by replacing row } j \text { in } V \text { by row } i \text { in } U .
\end{aligned}
$$

For example, in an obvious notation,

$$
\begin{gathered}
\phi_{12}=\left|\begin{array}{lll}
u_{11} & u_{12} & u_{13} \\
v_{11} & v_{12} & v_{13} \\
u_{31} & u_{32} & u_{33}
\end{array}\right|, \\
z_{1}=\operatorname{det} U, \quad z_{2}=\operatorname{det} V .
\end{gathered}
$$

The compound matrix equations can be derived by a number of different procedures; the authors used the systematic procedure in [3]. The resulting differential equations are listed here for completeness. We denote the 20 components of $z$ by $z_{1}, \ldots, z_{20}$.

$$
\begin{array}{ll}
z_{1}^{\prime}=z_{5}, & z_{2}^{\prime}=a_{41} z_{12}+a_{43} z_{18}+a_{61} z_{14}+2 z_{17} \\
z_{3}^{\prime}=a_{41} z_{1}-2 z_{9}, & z_{4}^{\prime}=-z_{3}-2 z_{10}, \\
z_{5}^{\prime}=-z_{4}+a_{43} z_{1}-2 z_{11}, & z_{6}^{\prime}=z_{9}+z_{17}, \\
z_{7}^{\prime}=-z_{6}+z_{10}-z_{14}, & z_{8}^{\prime}=-z_{7}+z_{11}, \\
z_{9}^{\prime}=a_{61} z_{1}+a_{65} z_{6}-z_{16}, & z_{10}^{\prime}=2 z_{1}-z_{9}+a_{65} z_{7}+z_{13}, \\
z_{11}^{\prime}=-z_{10}+a_{65} z_{8}, & z_{12}^{\prime}=z_{15}-2 z_{8}, \\
z_{13}^{\prime}=a_{43} z_{10}+a_{61} z_{14}+z_{16}+2 z_{17}, & z_{14}^{\prime}=-a_{43} z_{7}+2 z_{12}-z_{13}+z_{17}, \\
z_{15}^{\prime}=a_{61} z_{8}+z_{18}, & z_{16}^{\prime}=-a_{61} z_{5}-a_{43} z_{9}+a_{41} z_{11}-a_{65} z_{17}+z_{19}, \\
z_{17}^{\prime}=a_{43} z_{6}-a_{41} z_{8}+2 z_{15}-z_{16}+z_{20}, & z_{18}^{\prime}=z_{2}+2 z_{6}-a_{61} z_{7} \\
z_{19}^{\prime}=-2 z_{3}+a_{61} z_{4}-a_{41} z_{10}-a_{65} z_{20}, & z_{20}^{\prime}=a_{41} z_{7}+2 z_{13}-z_{19} .
\end{array}
$$

\subsection{Initial conditions and shooting}

In addition to transforming the block operator matrix into a system of differential equations, thence into the compound matrix system, one must also transform the boundary conditions associated with the block operator problem into initial conditions for shooting with the compound matrix method. The boundary conditions, which, in the original Ekman block operator form, are

$$
y(a)=y^{\prime}(a)=z(a)=0,
$$


transform firstly to the condition that the $6 \times 3$ matrix solution $Y_{a}$ of the $6 \times 6$ system $Y^{\prime}=A(x, \lambda) Y$, has the form

$$
Y_{a}(0, \lambda)=\left(\begin{array}{lll}
0 & 0 & 0 \\
0 & 0 & 0 \\
1 & 0 & 0 \\
0 & 1 & 0 \\
0 & 0 & 0 \\
0 & 0 & 1
\end{array}\right)
$$

up to post-multiplication by an invertible $6 \times 6$ matrix which we may choose to be the identity. It is now clear from the definitions of the $\phi_{i j}$ and $\psi_{i j}$ in $(9 \cdot 2)$ and $(9 \cdot 3)$ that only $\psi_{32}=z_{19}$ is nonzero at $x=a$. The precise non-zero value which we assign to $z_{19}(a)$ is unimportant, so the initial condition we use for forward shooting with the compound matrix system is

$$
z_{j}(a)= \begin{cases}0, & j \neq 19, \\ 1, & j=19\end{cases}
$$

Similar considerations hold for backward shooting. Following the results of Section 8 we can assume that the singular problem on $[a, \infty)$ has been replaced by a regular one on $[a, b]$ for some $b>a$. There will be regular boundary conditions at $x=b$, which will transform into boundary conditions on the solutions of the $6 \times 6$ system $Y^{\prime}=A(x, \lambda) Y$. There will be a $6 \times 3$ matrix solution $Y_{b}$ of the system $Y^{\prime}=A(x, \lambda) Y$ whose columns span the space of solutions of this system satisfying the boundary condition at $x=b$; and, finally, a compound matrix system associated with $Y_{b}$, whose differential equation is precisely $(9 \cdot 4)$ but whose initial condition is specified at $x=b$ rather than at $x=a$.

In order to determine whether or not a given $\lambda \in \mathbb{C}$ is an eigenvalue of the regularized problem over $[a, b]$ one must determine whether or not there exist nontrivial vectors $\mathbf{c}$ and $\mathbf{d}$ such that $Y_{a}(x, \lambda) \mathbf{c}=Y_{b}(x, \lambda) \mathbf{d}$ for one (and hence all) $x \in[a, b]$. The existence of such vectors guarantees the existence of a nontrivial solution of $Y^{\prime}=A(x, \lambda) Y$ which satisfies the boundary conditions both at $a$ and at $b$. The existence of such $\mathbf{c}$ and $\mathbf{d}$ is equivalent to the requirement that

$$
\operatorname{det}\left(Y_{a}(x, \lambda) Y_{b}(x, \lambda)\right) \equiv 0 .
$$

Let $z^{(a)}$ denote the solution of $(9 \cdot 4)$ subject to the boundary condition $(9 \cdot 5)$ and let $z^{(b)}$ denote the solution subject to the corresponding condition at $x=b$. The determinant on the left-hand side of (9.6) can be expressed in terms of $z^{(a)}(x)$ and $z^{(b)}(x)$ by Laplace expansion. It turns out that

$$
\begin{aligned}
\operatorname{det}\left(Y_{a}(x, \lambda) Y_{b}(x, \lambda)\right)= & z_{1}^{(a)} z_{2}^{(b)}-z_{1}^{(b)} z_{2}^{(a)}+z_{18}^{(a)} z_{5}^{(b)}-z_{18}^{(b)} z_{5}^{(a)}+z_{19}^{(a)} z_{8}^{(b)}-z_{19}^{(b)} z_{8}^{(a)}+z_{20}^{(a)} z_{11}^{(b)} \\
& -z_{20}^{(b)} z_{11}^{(a)}+z_{15}^{(a)} z_{4}^{(b)}-z_{15}^{(b)} z_{4}^{(a)}+z_{16}^{(a)} z_{7}^{(b)}-z_{16}^{(b)} z_{7}^{(a)}+z_{17}^{(a)} z_{10}^{(b)}-z_{17}^{(b)} z_{10}^{(a)} \\
& +z_{12}^{(a)} z_{3}^{(b)}-z_{12}^{(b)} z_{3}^{(a)}+z_{14}^{(a)} z_{9}^{(b)}-z_{14}^{(b)} z_{9}^{(a)}+z_{13}^{(a)} z_{6}^{(b)}-z_{13}^{(b)} z_{6}^{(a)} . \quad(9 \cdot 7)
\end{aligned}
$$

Thus if we integrate the differential equations for the compound matrix variables $z^{(a)}$ and $z^{(b)}$ from their known initial values at $a$ and at $b$ respectively, we can compute the function $\operatorname{det}\left(Y_{a}(x, \lambda) Y_{b}(x, \lambda)\right)$ for any fixed $x \in[a, b]$. The zeros of this function 
in the $\lambda$ plane are the eigenvalues and can be located using any reliable process for finding the zeros of an analytic function.

In practice, certain implementational precautions are needed. The value of $\operatorname{det}\left(Y_{a}(x, \lambda) Y_{b}(x, \lambda)\right)$ can be large (we encountered values of the order of $10^{170}$ during numerical experiments). A procedure for dealing with this problem is described in [14]: instead of computing $\operatorname{det}\left(Y_{a}(x, \lambda) Y_{b}(x, \lambda)\right)$ one must compute $\Gamma \in \mathbb{R}$ and $\omega \in \mathbb{C}$, $|\omega| \approx 1$, such that

$$
\operatorname{det}\left(Y_{a}(x, \lambda) Y_{b}(x, \lambda)\right)=\exp (\Gamma) \omega
$$

\section{Examples}

Numerics on the Ekman boundary layer problem have usually been executed using simple finite difference schemes [20] which give satisfactory results on regular problems where only modest precision is required. In this section we compare results from the compound matrix method described in Section 9 with results obtained by Lilly [20] on a simple regular problem, then pass to a singular problem for which the results on spectral inclusion and spectral exactness in Section 8 are important. In particular, we examine how well the essential spectrum of a singular problem is approximated in practice by eigenvalues of a regular problem.

\subsection{Example 1: approximation of eigenvalues in the left half-plane}

We have already seen in Section 3 that the essential spectrum of the Ekman problem lies entirely in the right half-plane. For linear stability analysis the important question is whether or not there are any eigenvalues in the left half-plane.

Rather than reproduce all the results of Lilly [20] we consider just one example from his paper: the classical problem of determining the critical Reynolds number. Figure 10 in $[20$, p. 486] shows, for the flow profiles

$$
U(x)=\cos \epsilon-\exp (-x) \cos (x+\epsilon), V(x)=-\sin \epsilon+\exp (-x) \sin (x+\epsilon), x \in[0, \infty),
$$

the dependence of the eigenvalues on $\epsilon$ and upon the Reynolds number $R$ for $\alpha=0.5$. In particular, this figure indicates that there should be no eigenvalues in the left halfplane for $R<R_{\text {crit }}$, where it appears that $R_{\text {crit }} \approx 120$. We repeated the computations of Lilly to obtain the neutral curve shown in Figure 1. The critical Reynolds number we obtained was approximately 114 . Considering that we were using a 6 th order method where Lilly used order 4 , that we used a truncated interval $[0,50]$ where he used intervals of between $[0,17]$ and $[0,35]$, and that our method had automatic stepsize control with a tolerance of $10^{-3}$ where Lilly used fixed stepsize with at most 35 steps, the agreement is remarkably good. To make it easier for others to reproduce these results we quote in Table 1 the numerical data behind the plot in Figure 1.

\subsection{Example 2: approximation of the essential spectrum}

We consider the Ekman boundary layer problem on $[0, \infty)$. As described in Section 3, the essential spectrum of this problem consists of the curve given parametrically by

$$
\lambda(t)=t^{2}+\alpha^{2}+\frac{2 i t}{\sqrt{t^{2}+\alpha^{2}}}, \quad t \in \mathbb{R}
$$


Table 1. Imaginary parts of eigenvalues for (10-1) for various parameter values. Real parts are all approximately zero

\begin{tabular}{cccccc}
\hline$\epsilon$ & $R$ & $\operatorname{Im}(\lambda)$ & $\epsilon$ & $R$ & $\operatorname{Im}(\lambda)$ \\
\hline 0.61 & 168.7 & -18.357 & -0.01 & 117.7 & 12.291 \\
0.60 & 166.0 & -17.631 & -0.05 & 120.2 & 14.478 \\
0.55 & 154.1 & -14.273 & -0.10 & 124.0 & 17.449 \\
0.50 & 144.2 & -11.271 & -0.15 & 128.0 & 20.639 \\
0.45 & 136.2 & -8.566 & -0.20 & 130.9 & 23.829 \\
0.40 & 129.6 & -6.074 & -0.23 & 131.6 & 25.606 \\
0.35 & 124.3 & -3.753 & -0.25 & 131.5 & 26.687 \\
0.30 & 120.3 & -1.548 & -0.27 & 130.9 & 27.660 \\
0.25 & 117.3 & 0.586 & -0.27 & 130.9 & 27.660 \\
0.20 & 115.2 & 2.688 & -0.30 & 129.7 & 29.014 \\
0.15 & 114.2 & 4.808 & -0.32 & 129.0 & 29.908 \\
0.10 & 114.2 & 6.993 & -0.33 & 128.8 & 30.380 \\
0.05 & 115.2 & 9.296 & -0.34 & 129.0 & 30.937 \\
0.01 & 116.7 & 11.257 & -0.35 & 129.1 & 31.470 \\
& & & -0.37 & 130.3 & 32.773 \\
& & & -0.40 & 134.6 & 35.379 \\
& & & -0.42 & 139.0 & 37.570 \\
\hline
\end{tabular}

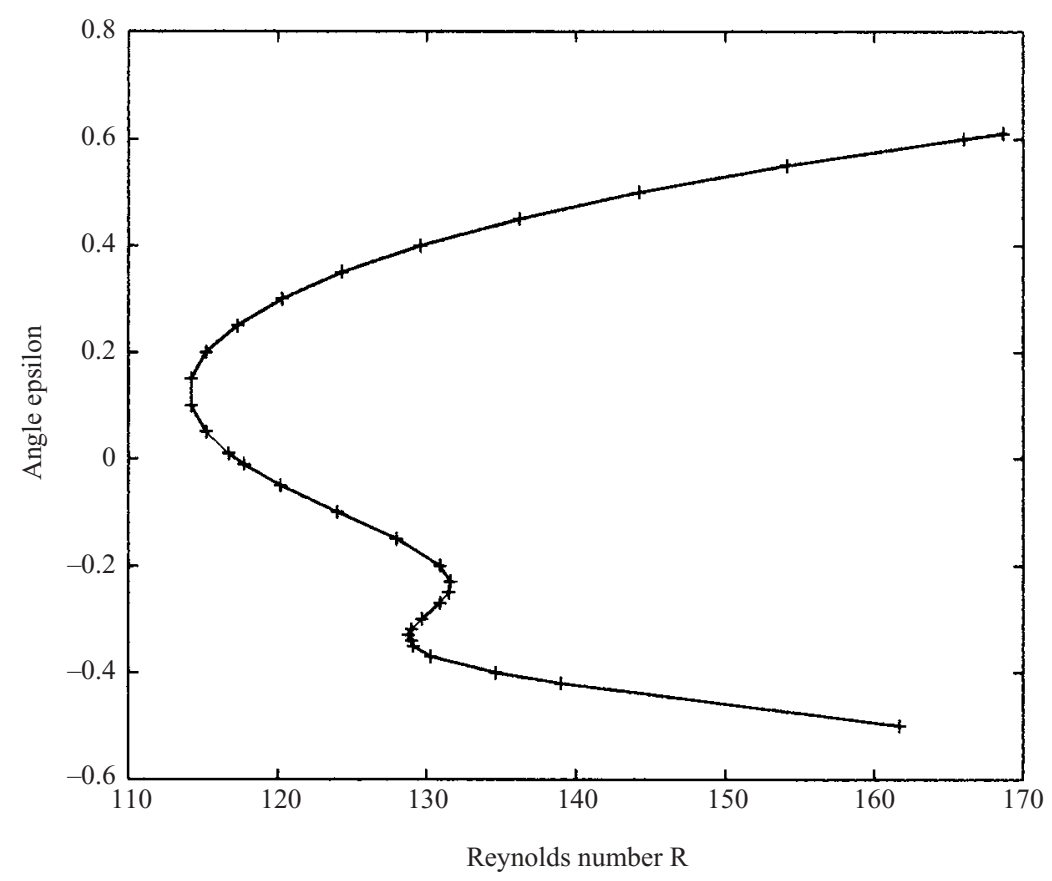

Fig. 1. Neutral curve for Ekman problem with $\alpha=0.5$. At the points on this curve, the leftmost eigenvalue of the Ekman problem lies on the imaginary axis in the complex plane.

provided the flow profiles $U$ and $V$ tend to zero sufficiently rapidly at infinity. If $V$ approaches some other constant value at infinity then the essential spectrum is shifted in the imaginary direction. 


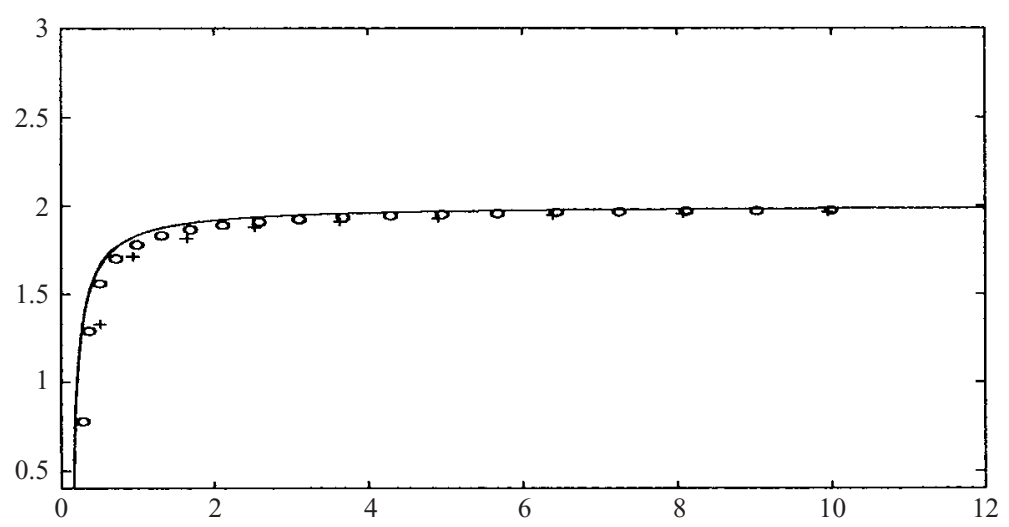

Fig. 2. Approximation of the essential spectrum by a regular problems on $[0,10]$ (plus signs) and $[0,20]$ (circles), here $\alpha=0.4$ and $U \equiv 0 \equiv V$.

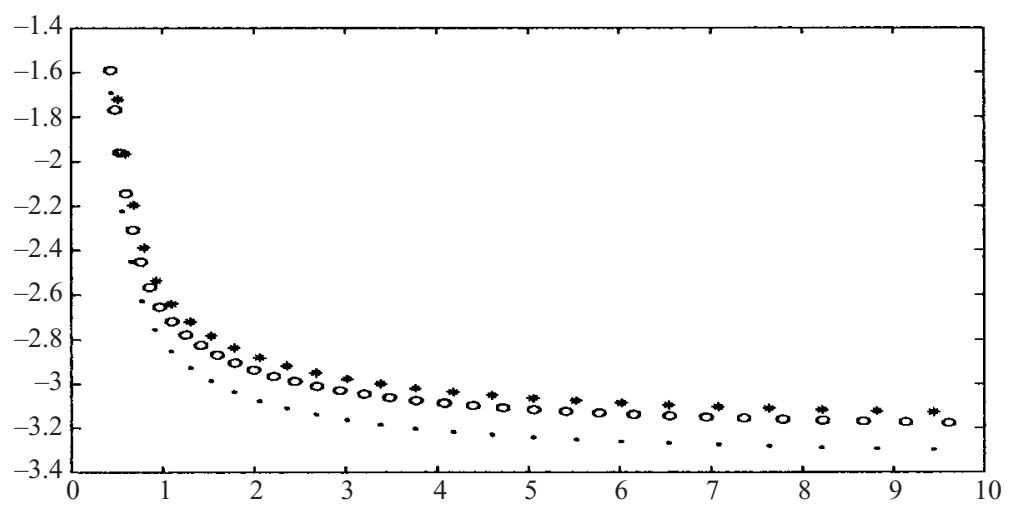

Fig. 3. Approximation of the essential spectrum by a regular problems. The lower branch is shown, rather than the upper branch which is shown in Fig. 2.

We considered first the case in which $U \equiv 0 \equiv V$, with $\alpha=0.4$. We replaced the singular problem by two regular ones, one posed on the interval $[0,10]$ and the other posed on $[0,20]$. The results are shown in Figure 2, where the continuous curve is a section of the exact essential spectrum, the ' + ' signs denote eigenvalues for the problem on $[0,10]$ and the circles denote eigenvalues for the problem posed over $[0,20]$. The longer interval has, as one would expect, more eigenvalues, and one observes by inspection of Figure 2 that they yield better approximations to the essential spectrum. Note that there are no spurious eigenvalues due to interval truncation in the region of the complex plane shown in Figure 2.

Secondly, we considered the problem with

$$
U(x)=\cos (\epsilon)-\exp (-x) \cos (x+\epsilon), \quad V(x)=-\sin (\epsilon)+\exp (-x) \sin (x+\epsilon),
$$

with $\alpha=0.4, \epsilon=0.27$ and $R=10$. We compared the results for a problem posed over $x \in[0,30]$ with problems posed over $x \in[0,40]$ and $x \in[20,50]$, using the artificial boundary conditions $y(X)=y^{\prime}(X)=y^{\prime \prime}(X)=0$ and $z(X)=0$, where $X=30, x=40$ or $X=60$ as appropriate. From the results of Section 8 we know that these will allow approximation of the spectrum. The results in Figure 3 show, however, that such naive boundary conditions may result in quite slow convergence 
of approximations to the spectrum. To get better results one must use boundary conditions based on the easily-computed asymptotics of the solutions $y$ and $z$ at infinity.

\section{Concluding remarks}

In this paper we have shown that simple arguments based on linear algebra may be used to reduce quite general block-operator eigenproblems to systems of differential equations with $\lambda$-rational coeffiecients. We have also shown that for the case of the Ekman problem on a half line with flow profiles in $L^{1}[0, \infty)$, the location of the essential spectrum can be obtained analytically in the form of a parametrized smooth curve. Moreover, for problems which can be reduced to systems of ODEs having some of the same qualitative properties as the system obtained from the Ekman problem, results on spectral inclusion and spectral exactness for regular approximations to the original problem can be obtained in spite of the fact that the problem is nonselfadjoint. Our approach to these results follows the Levinson asymptotics described in Eastham [10] and the approach to Sims Case 1 problems in [7] rather than the more classical approach of, e.g., Markowich [21] (which, although valid here, would not easily generalize to more complicated problems). Numerical results indicate that while the results on spectral inclusion guarantee the existence of approximations to spectral points, the convergence may be quite slow with a naïve choice of boundary conditions.

Acknowledgements. The authors are grateful to the EPSRC for support which enabled them to work together in Leicester during several summers, most recently under VF grant GR/N26159.

\section{REFERENCES}

[1] V. Adamyan, H. Langer and R. Mennicken. Spectral components of selfadjoint block operator matrices with unbounded entries. Math. Nachr. 178 (1996), 43-80.

[2] V. Adamyan, R. Mennicken and J. Saurer. On the discrete spectrum of some selfadjoint operator matrices. J. Operator Theory 39 (1998), 3-41.

[3] L. Allen and T. J. Bridges. Numerical exterior algebra and the compound matrix method. Technical Report, Department of Mathematics and Statistics, University of Surrey (July, 2000).

[4] F. V. Atkinson, H. Langer, R. Mennicken and A. Shkalikov. The essential spectrum of some matrix operators. Math. Nachr. 167 (1994), 5-20.

[5] P. B. Bailey, W. N. Everitt, J. Weidmann and A. ZetTl. Regular approximation of singular Sturm Liouville problems. Results Math. 23 (1993), 3-22.

[6] B. M. Brown, L. Greenberg and M. Marletta. Convergence of regular approximations to the spectra of singular fourth order Sturm-Liouville problems. Proc. Royal Soc. Edinburgh Sect. A128 (1998), 907-944.

[7] B. M. Brown and M. Marletta. Spectral inclusion and spectral exactness for non-self-adjoint Sturm-Liouville problems. Proc. Royal Soc. Lond. A457 (2001), 117-139.

[8] E. B. Davies. Pseudospectra: the harmonic oscillator and complex resonances. Proc. Royal Soc. Lond. A455 (1999), 585-599.

[9] P. G. Drazin and W. H. ReID. Hydrodynamic Stability (Cambridge University Press, 1981).

[10] M. S. P. Eastham. Asymptotic Solution of Linear Differential Systems (Oxford University Press, 1989).

[11] D. Edmunds and W. Evans. Spectral Theory and Differential Operators (Clarendon Press, Oxford, 1987).

[12] A. J. FALLER. An experimental study of the instability of the laminar Ekman boundary layer. J. Fluid Mech. 15 (163), 560-576.

[13] F. Gilbert and G. E. Backus. Propogator matrices in elastic wave and vibration problems. Geophysics 31 (1966), 326-332. 
[14] L. Greenberg and M. Marletta. Numerical solution of non-selfadjoint Sturm-Liouville problems and related systems. SIAM J. Numer. Anal. 38 (2001), 1800-1845.

[15] V. Hardt, R. Mennicken and S. Naboko. Differential operators of mixed order and applications to 1-dimensional MHD problems I. Math. Nachr. 205 (1999), 19-68.

[16] D. B. Hinton and J. K. Shaw. On Titchmarsh-Weyl $m(\lambda)$-functions for Hamiltonian systems. J. Differential Equations 40 (1981), 316-342.

[17] J. Lahmann and M. Plum. On the spectrum of the Orr-Sommerfeld equation on the semiaxis. Math. Nachr. 216 (2000), 145-153.

[18] H. Langer and C. Tretter. Spectral properties of the Orr-Sommerfeld problem. Proc. Roy. Soc. Edinburgh A127 (1997), 1245-1261.

[19] A. Lifschitz. Magnetohydrodynamics and Spectral Theory (Kluwer Academic Publishers, 1988).

[20] D. K. Lilly. On the Instability of the Ekman Boundary Flow. J. Atmospheric Sci. 23 (1966), 481-494.

[21] P. A. Markowich. Eigenvalue problems on infinite intervals. Math. Comp. 39 (1982), 421-444.

[22] B. S. NG and W. H. ReID. An initial value method for eigenvalue problems using compound matrices. J. Comput. Phys. 30 (1979), 125-136.

[23] M. Reed and B. Simon. Methods of Modern Mathematical Physics; I: Functional Analysis (Academic Press, 1972).

[24] A. Shkalikov. How to define the Orr-Sommerfeld operator? Moscow Univ. Math. Bull. 53 (1998), 4, 1-7.

[25] G. Stolz and J. Weidmann. Approximation of isolated eigenvalues of ordinary differential operators. J. Reine Angew. Math. 445 (1993), 31-44.

[26] G. Stolz and J. Weidmann. Approximation of isolated eigenvalues of general singular ordinary differential operators. Results Math. 28 (1995), 345-358.

[27] B. Straughan. Explosive Instabilities in Mechanics (Springer-Verlag, 1998). 